\title{
Novel treatment strategies for the protection of the preterm brain
}

Citation for published version (APA):

Ophelders, D. R. M. G. (2017). Novel treatment strategies for the protection of the preterm brain: rebalancing inflammation and regeneration. [Doctoral Thesis, Maastricht University]. Datawyse / Universitaire Pers Maastricht. https://doi.org/10.26481/dis.20170317do

Document status and date:

Published: 01/01/2017

DOI:

10.26481/dis.20170317do

Document Version:

Publisher's PDF, also known as Version of record

Document license:

Unspecified

\section{Please check the document version of this publication:}

- A submitted manuscript is the version of the article upon submission and before peer-review. There can be important differences between the submitted version and the official published version of record.

People interested in the research are advised to contact the author for the final version of the publication, or visit the DOI to the publisher's website.

- The final author version and the galley proof are versions of the publication after peer review.

- The final published version features the final layout of the paper including the volume, issue and page numbers.

Link to publication

\footnotetext{
General rights Owners
rights.

- You may freely distribute the URL identifying the publication in the public portal. please follow below link for the End User Agreement:

www.umlib.nl/taverne-license

Take down policy

If you believe that this document breaches copyright please contact us at:

repository@maastrichtuniversity.nl

providing details and we will investigate your claim.
}

Copyright and moral rights for the publications made accessible in the public portal are retained by the authors and/or other copyright owners and it is a condition of accessing publications that users recognise and abide by the legal requirements associated with these

- Users may download and print one copy of any publication from the public portal for the purpose of private study or research.

- You may not further distribute the material or use it for any profit-making activity or commercial gain

If the publication is distributed under the terms of Article $25 \mathrm{fa}$ of the Dutch Copyright Act, indicated by the "Taverne" license above, 


\section{Novel treatment strategies for the}

protection of the preterm brain

Re-balancing inflammation and regeneration

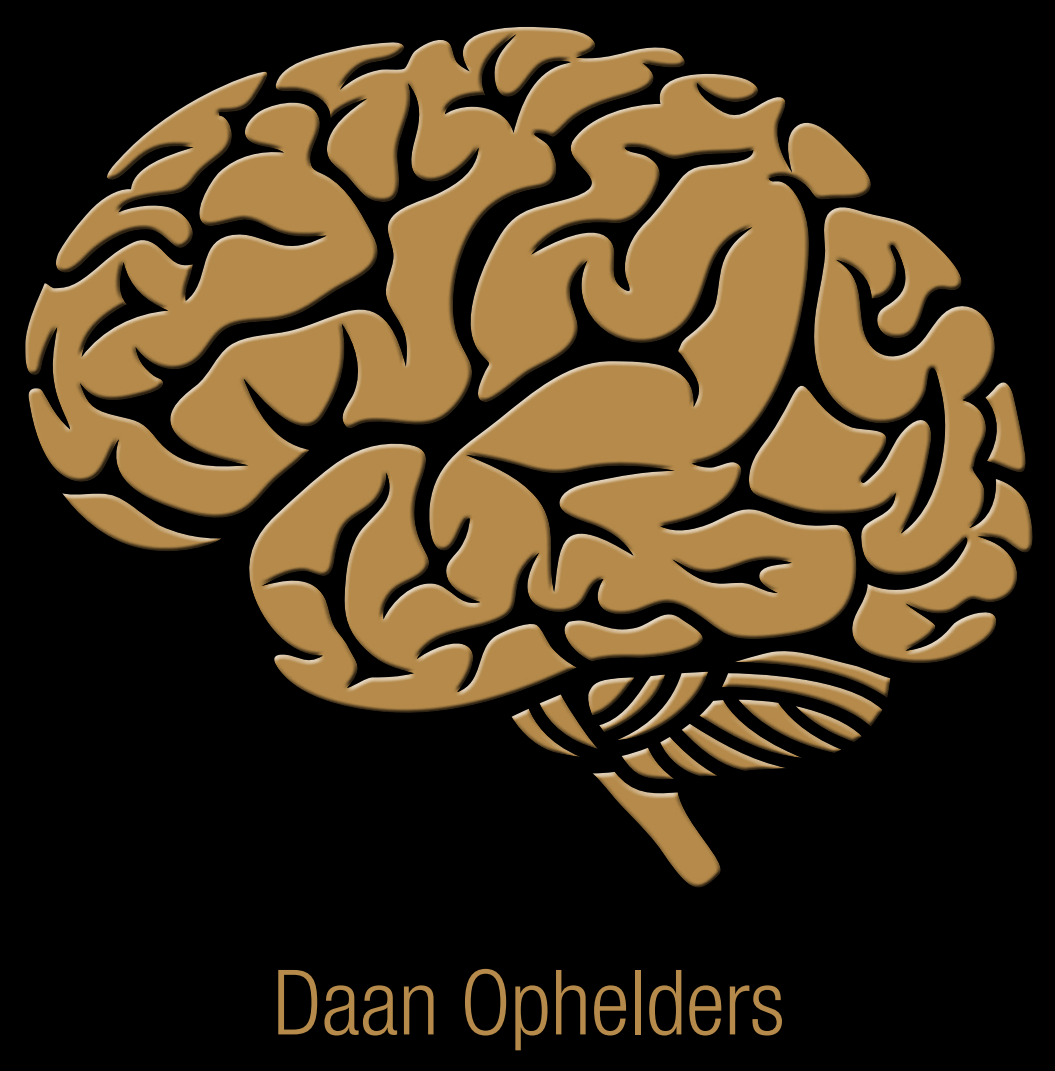




\title{
Novel treatment strategies for the protection of the preterm brain
}

\author{
Re-balancing inflammation and regeneration
}

\author{
PROEFSCHRIFT
}

ter verkrijging van de graag van doctor aan de Universiteit Maastricht, op gezag van Rector Magnificus, Prof. Dr. Rianne M. Letschert

volgens het besluit van het College van Decanen,

in het openbaar te verdedigen

op donderdag 16 maart 2017 om 10.00 uur

door

Daan R.M.G. Ophelders

geboren op 8 juli 1983 te Venlo 


\section{Promotor}

Prof. Dr. Boris W. Kramer

\section{Copromotor}

Dr. Tim G.A.M. Wolfs

Dr. Reint K. Jellema

\section{Beoordelingscommissie}

Prof. Dr. Yasin Temel (voorzitter)

Dr. Bobbi Fleiss, King's College London

Prof. Dr. Niels Hellings, Universiteit Hasselt

Prof. Dr. Chris Reutelingsperger

Dr. Jeroen Vermeulen 


\section{Abbreviations}

aEEG

AMPA

ANOVA

$A P C$

ATP

BCA

BPD

BRS

BSA

BW

C. albicans

CD

cEEG

CFU

$\mathrm{Cl}$

CNPase

CNS

$\mathrm{CP}$

CSF

$D A B$

DAMPS

DTT

ECG

EEG

ELISA

EVS

$\mathrm{F}$

FDR

FHR

FIRS

GA

GFAP

GVHD

$H \& E$

$\mathrm{H}_{2} \mathrm{O}_{2}$

$\mathrm{HCl}$

$\mathrm{HI}$ amplitude-integrated electroencephalogram

$\alpha$-amino-3-hydroxy-5-methyl-4-isoxazole-propionic acid

analysis of variance

antigen presenting cells

adenosine triphosphate

bicinchoninic acid

bronchopulmonary dysplasia

baroreceptor reflex sensitivity

bovine serum albumin

body weight

Candida albicans

cluster of differentiation

conventional electroencephalogram

colony forming units

confidence interval

2',3'-Cyclic-nucleotide 3'-phosphodiesterase

central nervous system

cerebral palsy

cerebrospinal fluid

3,3'-diaminobenzidine

damage-associated molecular patterns

dithiothreitol

electrocardiogram

electroencephalogram

enzyme-linked immunosorbent assay

extracellular vesicles

Fluconazole

false discovery rate

fetal heart rate

fetal immune response syndrome

gestational age

glial fibrillary acidic protein

graft-versus-host-disease

hematoxylin \& eosin

hydrogen peroxide

hydrochloric acid

hypoxia-ischemia 


\begin{tabular}{|c|c|}
\hline HIE & hypoxic-ischemic encephalopathy \\
\hline HLA & human leukocyte antigen \\
\hline HPA & hypothalamic-pituitary-adrenal \\
\hline HRP & horseradish peroxidase \\
\hline $\mid \mathrm{A}$ & intra-amniotic \\
\hline IBA-1 & ionized calcium-binding adaptor molecule-1 \\
\hline IFNY & interferon gamma \\
\hline IL & interleukin \\
\hline IP & intra-peritoneal \\
\hline IQR & interquartile range \\
\hline IR & immunoreactivity \\
\hline MABP & mean arterial blood pressure \\
\hline MAPC & multipotent adult progenitor cell \\
\hline MBP & myelin basic protein \\
\hline $\mathrm{MHC}$ & major histocompatibility complex \\
\hline MPAQ & Maastricht-programmable acquisition system \\
\hline MPO & myeloperoxidase \\
\hline MSC & mesenchymal stromal cells \\
\hline MSC-EVs & mesenchymal stromal cell-derived extracellular vesicles \\
\hline $\mathrm{NaCl}$ & sodium chloride \\
\hline NEC & necrotizing enterocolitis \\
\hline $\mathrm{NICU}$ & neonatal intensive care unit \\
\hline NiDAB & nickel sulfate-diaminobenzidine \\
\hline NMDA & N-methyl-D-Aspartate \\
\hline NTA & Nanoparticle Tracking Analyses \\
\hline Olig2 & oligodendrocyte transcription factor 2 \\
\hline ovRPS15 & ovine 40 S ribosomal protein S15 \\
\hline PAMPs & pathogen-associated molecular patterns \\
\hline PAS & periodic acid-schiff \\
\hline PBS & phosphate buffered saline \\
\hline PBST & tween in phosphate buffered saline \\
\hline PEG & polyethylene glycol \\
\hline PPROM & preterm premature rupture of the membranes \\
\hline preOLs & oligodendrocyte precursors \\
\hline PRR & pattern recognition receptors \\
\hline PVDF & polyvinylidene difluoride \\
\hline PVL & periventricular leukomalacia \\
\hline PVWM & periventricular white matter \\
\hline RNA & ribonucleic acid \\
\hline RNS & reactive nitrogen species \\
\hline ROP & retinopathy of prematurity \\
\hline
\end{tabular}


ROS

reactive oxygen species

RT-qPCR

real time quantitative polymerase chain reaction

SAL

saline

SBP

systolic blood pressure

SCWM

subcortical white matter

$S D$

standard deviation

SD-RR

standard deviation of the mean R-R interval

SDS

sodium dodecyl sulfate

SD-SBP

standard deviation of the mean systolic blood pressure

SDS-PAGE

sodium dodecyl sulfate polyacrylamide gel electrophoresis

SEM

T cell standard error of the mean

Treg

T-lymphocyte

TBS

regulatory T-lymphocyte

TMB

tris-buffered saline

TNF $\alpha$

3,3',5,5'-tetramethylbenzidine

tumor necrosis factor alpha

Tsg101

tumor susceptibility gene 101

UCO

umbilical cord occlusion

WM

white matter 



\section{Contents}

Abbreviations

Chapter 1 General Introduction

Chapter 2 Clinical-grade Multipotent Adult Progenitor Cells for HypoxicIschemic Injury in the Preterm Brain

Chapter 3 Mesenchymal Stromal Cell-derived Extracellular Vesicles Protect the Fetal Brain after Hypoxia-Ischemia

Chapter 4 Neuroinflammation and Structural Injury of the Fetal Ovine Brain Following Intra-amniotic Candida albicans Exposure

Chapter 5 General Discussion 95

Summary

Samenvatting

Valorization

Acknowledgements

Curriculum vitae

Publications

129 



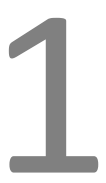

General Introduction 



\section{PRETERM BIRTH}

Preterm birth is defined as birth before 37 weeks of gestation and is the leading cause of perinatal mortality and morbidity in developed countries. ${ }^{1}$ In the Netherlands, approximately $7.5 \%$ of all infants are born preterm. ${ }^{2}$ In contrast to increasing numbers of preterm births world-wide, in the Netherlands, preterm birth numbers have stagnated over the last years. ${ }^{2}$

Survival rates following preterm birth have sharply increased over the last decades. This increase can be attributed to improved early perinatal and neonatal care, such as improved prenatal diagnostics, the use of antenatal corticosteroids, postnatal administration of exogenous surfactant, centralization of perinatal care, and advanced ventilator strategies. ${ }^{3,4}$ Upon birth, almost all preterm neonates require medical care to support function and further development of their immature organs. The kind and intensity of medical care for preterm babies is mostly determined by their gestational age and corresponding degree of developmental immaturity of their organs. This immaturity results in the incapacity to deal with the challenges of life outside the uterus, mostly oxygen and inflammation, and the underlying cause of preterm birth. Despite improved medical care, preterm birth remains associated with development of diseases of prematurity such as necrotizing enterocolitis (NEC), bronchopulmonary dysplasia (BPD), retinopathy of prematurity (ROP), and periventricular leukomalacia $(P V L)$ resulting in cerebral palsy $(C P){ }^{5}$ From these diseases, injury to the preterm brain (PVL and $\mathrm{CP}$ ) is the biggest contributor to long-term morbidity in preterm infants. ${ }^{6}$ During gestation, complex differentiation and maturation processes contribute to fetal brain development in a spatial- and time-dependent fashion. For instance, white matter myelination follows a caudo-rostral gradient and progresses from the center to the periphery. ${ }^{7,8}$ Preterm infants are born during this critical developmental period, in which the fetal brain is particularly vulnerable to exogenous and endogenous stressful events, such as intra-amniotic infection/inflammation and hypoxia-ischemia. These events might affect normal brain ontogenesis and may cause irreversible injury to the brain.

\section{CAUSES OF PRETERM BIRTH}

Preterm birth can be subdivided in distinct categories: (1) medically indicated preterm birth in which maternal and/or fetal conditions prompt an immediate intervention at preterm gestational ages (preeclampsia, intrauterine growth restriction, and placental abruption conditions) and (2) spontaneous preterm birth with intact membranes; or (3) preterm premature rupture of the membranes (PPROM). ${ }^{1}$ Many different causes can trigger spontaneous premature uterine contractions leading to preterm birth, including 
(1) activation of the maternal/fetal hypothalamic-pituitary-adrenal (HPA) axis as a result of maternal and/or fetal stress, (2) infection and inflammation, (3) hypoxia-ischemia and (4) uterine over distension (for instance during a multiple pregnancy). ${ }^{1,9-11}$ From these causes infection and inflammation and hypoxia-ischemia are most frequently diagnosed and will be subject of this thesis., ${ }^{1,-11}$

The uterine environment has long been considered to be a sterile environment. With the availability of advanced microbiological methodologies, we are now aware that numerous microbial organisms colonize the uterine environment, some of which have been causally linked to preterm birth. ${ }^{12}$ Importantly, bacterial colonization of the amniotic cavity does not necessarily induce preterm birth. ${ }^{13-16}$ Timing of bacterial colonization of the amniotic cavity rather than colonization itself is a key factor for the risk of preterm birth. ${ }^{14,16}$ Therefore, the percentage of diagnosed intra-uterine infections is much higher in very preterm infants compared to near term and term infants. $^{10,17-19}$

Nonetheless, bacterial colonization without induction of preterm birth remains sufficient to cause adverse fetal development and cause injury to fetal organ systems. ${ }^{16}$ Such an intra-amniotic infection induces an inflammatory response of the chorionic and amniotic membranes, which is referred to as chorioamnionitis. Various routes have been described by which microorganisms gains access to the amniotic cavity: (1) ascending from the vagina and the cervix, which is the most common pathway; (2) hematogenous dissemination through the placenta; (3) accidental introduction at the time of invasive procedures (i.e. amniocentesis); (4) retrograde spread through the fallopian tubes; and (5) asymptomatic endometrial colonization before pregnancy. ${ }^{20}$

A wide variety of microorganisms, comprising bacteria, viruses, and fungi/yeasts have been identified in the amniotic fluid and membranes of preterm infants. ${ }^{21-23}$ It is unclear during which part of conception, implantation and gestation intra-uterine infection or microbial invasion occurs. Most women do not show clinical symptoms of chorioamnionitis which is referred to as silent chorioamnionitis and is only diagnosed at birth by histological examination. A small proportion of pregnant women with preterm birth, however, show clinical signs of chorioamnionitis such as maternal fever, uterine fundal tenderness, maternal tachycardia, fetal tachycardia, and purulent or foul amniotic fluid. Upon diagnosis of this clinical chorioamnionitis preterm birth is often medically indicated, whereas asymptomatic chorioamnionitis often results in spontaneous preterm birth.

The mechanisms by which intrauterine infection / inflammation lead to preterm birth comprise activation of the innate immune system: recognition of pathogen-associated molecular patterns (PAMPs), expressed by invading microorganisms, by pattern recognition receptors (i.e. toll-like receptors) elicits the release of pro-inflammatory mediators such as interleukin (IL)-8, IL-1 $\beta$, and tumor necrosis factor (TNF)- $\alpha .{ }^{1}$ Microbial 
endotoxins and pro-inflammatory cytokines stimulate the production of prostaglandins, other pro-inflammatory mediators, and matrix-degrading enzymes. Prostaglandins stimulate uterine contractility, whereas degradation of extracellular matrix in the fetal membranes leads to PPROM. ${ }^{1,10}$

When the fetus is directly exposed to intra-amniotic inflammation / infection (i.e. skin contact or swallowing and breathing movements in utero or via the placental-fetal circulation), recognition of PAMPs by the fetal innate immune system might result in an elevation of levels of pro-inflammatory cytokines. ${ }^{24,25}$ This response is referred to as Fetal Immune Response Syndrome (FIRS). ${ }^{24}$ Intrauterine infection/inflammation is causally involved in the induction of organ injury, including the brain. Although the central nervous system is an immune-privileged site, separated from the systemic circulation by the blood brain barrier, microglia, the resident immune cells of the central nervous system, can sense inflammatory signals in the systemic circulation through: (1) interaction of systemic molecules with cerebral immune cells in the circumventricular organs: areas that are devoid of a blood brain barrier ${ }^{26-29}$; (2) Direct access of inflammatory mediators or cells into the central nervous system facilitated by breakdown of the blood brain barrier ${ }^{26-29}$; (3) interactions of systemic molecules with endothelial cells resulting in release of inflammatory mediators (i.e. prostaglandins) at the basal side into the brain parenchyma. ${ }^{26-29}$

\section{HYPOXIA-ISCHEMIA}

Fetal hypoxia-ischemia ( $\mathrm{HI})$ is the result of impaired gas exchange between mother and fetus. Causes for fetal $\mathrm{HI}$ are inadequate placental perfusion due to maternal complications, impaired umbilical cord blood flow due to mechanical compression, rupture or prolapse of the umbilical cord. In the case of hypoxia-ischemia preterm birth is mostly medically indicated.

Regardless of the cause of the hypoxic insult, cardiac and vascular compromise will ultimately occur when hypoxia is prolonged. This results in hypotension, ischemia and anaerobic metabolism. Ischemia therefore is both the cause and a consequence of hypoxia leading to diminished delivery of nutrients and impaired removal of metabolites. The process of hypoxia-ischemia resulting in energy failure and subsequent brain injury is a process that evolves over time and can be subdivided into two main phases $^{30,31}:$ (1) primary phase of injury and (2) secondary phase of injury.

During the initial phase cerebral oxidative metabolism is severely compromised resulting in depletion of adenosine triphosphate (ATP) and uncontrolled membrane polarization allowing for cellular influx of calcium and subsequent proteolytic degradation of multiple cellular structures. Neuronal release of glutamate accompanied 
by impaired removal of extracellular glutamate by astrocytes causes its accumulation and activation of $\mathrm{N}$-methyl-D-Aspartate (NMDA) channels, $\alpha$-amino-3-hydroxy-5methyl-4-isoxazole-propionic acid (AMPA) and kainate receptors, resulting in acute cell swelling, production of reactive oxygen (ROS) and reactive nitrogen species (RNS), and NMDA receptor-mediated seizure activity. ${ }^{30-32}$

After reperfusion, the initial induced accumulation of excitatory amino acids resolves with apparent recovery cerebral oxidative metabolism. Although short in time, the neurotoxic cascade is largely inhibited during this period, thereby providing a "therapeutic window of opportunity" for neuroprotective therapies. ${ }^{33}$

Although restoration of blood and oxygen supply to the brain is necessary to limit the hypoxic-ischemic brain damage, reperfusion exacerbates cell injury in the secondary phase. Intracellular increase of calcium during hypoxia-ischemia and increased oxygen levels during reperfusion are involved in formation and accumulation of ROS and RNS. Production of free radicals results in oxidation of cellular components such as membrane lipids, proteins, and DNA, leading to mitochondrial injury cellular damage, and cell death by either apoptosis or necrosis. Whereas apoptosis is an orchestrated process of cell death that causes negligible effects on the extracellular environment, necrosis caused disruption of cell-membrane integrity and leakage of cellular components that function as danger-associated molecular patterns (DAMPs) to trigger a sterile (non-infectious) inflammatory response. ${ }^{34}$

Despite differences in etiology (infectious versus sterile inflammation), microglia are at the convergence point in the neuroinflammatory cascade leading to preterm brain injury. ${ }^{29,}{ }^{35}$ Microglia are the resident macrophages of the central nervous system, and have the ability to recognize a wide range of signals (i.e. PAMPs and DAMPs) that indicate a threat to the structural and functional integrity of the CNS through various pattern recognition receptors (PRRs), glutamate receptors, and purinergic receptors, resulting in activation. ${ }^{36-38}$ Microglial activation is necessary for destruction of microorganisms, clearance of cellular debris and repair of tissue. However, uncontrolled activation of microglia can cause damage to neighboring neurons and oligodendrocytes. Upon activation, microglia change their morphology from a ramified to a hyperramified and subsequent amoeboid morphology. ${ }^{38}$ These morphological changes are accompanied by a robust production of pro-inflammatory cytokines, reactive oxygen and nitrogen species, excitotoxic molecules, and proteolytic enzymes. ${ }^{38,} 39$ Moreover, microglia act as antigen presenting cells (APCS) through upregulation of HLA molecules, co-stimulation and cytokine production, thus providing signals necessary for activation of T-lymphocytes (T cells). ${ }^{40}$

Astrocytes also play a crucial role in the brain. These cells are key in regulating secretion/absorption of neurotransmitters, transmission of electrical impulses, maintenance of the blood-brain barrier, providing nutrients to neuronal tissue and are 
involved in glial scar formation after damage. Moreover, reactive astrocytes are an important source of pro-inflammatory cytokines that affect neurons and oligodendrocytes. ${ }^{41,42}$

Under inflammatory conditions, the blood-brain barrier is disrupted and CNS immune privilege is greatly undermined, allowing for active recruitment of circulating leukocytes, including monocyte-derived macrophages, neutrophils and $\mathrm{T}$ cells. These systemic immune cells contribute to the neuroinflammatory response and subsequent injury of the preterm brain. ${ }^{43-45}$

Preterm brain injury is mainly characterized to injury of the cerebral white matter. Myelination of axons by mature oligodendrocytes is essential for optimal signal transduction and subsequent brain function and development. During gestation, cerebral white matter is predominantly populated by oligodendrocyte precursors (preOLs) that develop towards mature, myelin producing, oligodendrocytes. At this stage preOLs are most vulnerable to excitotoxic, oxidative, and inflammatory injury, resulting in maturational arrest or cell death and subsequent hypomyelination, characteristic for preterm brain injury. ${ }^{6,46-48}$

During maturation preOLs have low levels of anti-oxidants, making them particularly sensitive for excessive production of free radicals. In addition, during the peak period of vulnerability maturing preOLs overexpress the AMPA receptor that is lacking the GluR2 subunit. Deficiency of this subunit makes the preOL calcium-permeable upon stimulation with glutamate, thereby causing intracellular generation of ROS/RNS. ${ }^{35}$

The main form of white matter injury is periventricular leukomalacia (PVL), which can be sub-divided into two forms. ${ }^{48}$ Focal (cystic) PVL is characterized by macroscopic necrotic lesions and loss of all cellular elements. These necrotic lesions evolve into cystic lesions that can be visualized by cranial ultrasound. However, the incidence of focal PVL has decreased tremendously and nowadays non-cystic PVL is the most common variant of PVL. ${ }^{6,48}$ Non-cystic PVL is characterized by diffuse spread of microscopic necrotic foci with concomitant astro- and microgliosis and a decrease in premyelinating oligodendrocytes. This decrease is often counteracted by an increase in oligodendrocyte progenitor cells. However, these cells often lack processes and do not seem to have the capacity to fully mature into myelin-producing oligodendrocytes. $6,46,48$

\section{GRAY MATTER}

Although white matter injury is the hallmark of preterm brain injury, accumulating evidence suggest an increasing role for gray matter in the outcomes of preterm infants suffering from preterm brain injury. ${ }^{6}$ Depending on the gestational age and injurious insult, reduction in growth of and significant neuronal loss in cortical and subcortical 
gray matter structures, including the basal ganglia, thalamus, hippocampus, and cerebellum are identified. ${ }^{49}$ In addition, subplate neurons, a transient cell population, required to establish thalamo-cortical projections, are vulnerable to prenatal insults. Disturbance of formation of thalamo-cortical projections is a critical risk factor for adverse neurocognitive outcomes. ${ }^{6,50,51}$

\section{DIAGNOSIS}

Imaging techniques are frequently used for the detection of white matter abnormalities during the early neonatal period. Although minimally invasive, weeks or more are required for structural injury to become evident on ultrasonography. As preterm brain injury often presents with clinical and electrophysiological seizures, assessment of brain function with electroencephalography (EEG) provides a powerful, sensitive and noninvasive tool for the assessment of brain injury in these infants. ${ }^{52-54}$

EEG records the cerebral electrical activity of the brain through electrodes attached to the scalp. Although, conventional EEG (CEEG) is the gold standard for evaluating the neurologic functional status of preterm neonates in the neonatal intensive care unit (NICU), it presents some difficulty because of the involved technical complexity, time demands, intermittent availability, interpretation specialty, and costs. ${ }^{55}$ Amplitudeintegrated EEG (aEEG), in contrast to $C E E G$, is a simplified method of continuous brain function monitoring that displays trended brain activity and is highly correlated with CEEG in monitoring background activities of both healthy and neurologically compromised neonate. ${ }^{52,56}$ aEEG consists of a single- or double-channel EEG that is recorded by three to five electrodes attached to the scalp. ${ }^{57}$ It continuously captures the general background pattern of the brain activity in real time for an extended period. Recordings can last for 24 hours or longer if needed. The electrode application and aEEG interpretation entail relatively short training and experience to master. The benefit of longer-term recordings by aEEG may be useful for capture of episodic seizure activity and evolving trends in background activity after acute injury and to monitor therapeutic responses. ${ }^{58}$

Besides assessment of electrocortical cortical and background activity with aEEG, analysis of activity of deep brain structures (i.e. brainstem) might provide an additional diagnostic tool for neonatal outcome as crucial basic functions (i.e. baroreceptor reflex) are located in this structure. The baroreflex is primarily responsible for short-term autonomic control of blood pressure and minimizes fluctuations in by increasing or decreasing heart rate, myocardial contractility, and arterial vascular resistance. ${ }^{59}$

Infants born preterm have a reduced baroreceptor reflex sensitivity (BRS) at birth compared to term-born infants. Moreover, preterm birth alters the normal maturation 
of BRS, with reduced BRS in preterm infants at term-equivalent age compared with newborn term infants. ${ }^{59}$

The clinical relevance of an impaired baroreceptor reflex function relates to the increased risk for developing additional brain injury by exposing the vulnerable developing cerebral vascular network to (large) fluctuations in blood pressure. In preterm infants, fluctuations associated with high blood pressure may disrupt the cerebral capillaries in the germinal matrix while periods of low blood pressure may cause localized hypoxia-ischemia of the watershed areas. ${ }^{60}$ Moreover, preterm infants display fluctuating pressure-passivity between systemic blood pressure and cerebral blood flow representing a considerably increased risk of cerebral hemorrhage or hypoxia. ${ }^{60}$

\section{TREATMENT STRATEGIES}

Following birth, all preterm infants receive supportive care for adequate respiratory support, optimizing blood gases and blood pressure, normalizing fluid-electrolyte balance and blood glucose levels, and, if necessary, antibiotic treatment and treatment of seizures. Preterm brain injury evolves over time in a period of hours to weeks, creating a window of opportunity. However, therapeutic options to improve the outcomes of preterm neonates suffering from brain injury are very limited.

Current approaches to treat the consequences of chorioamnionitis focus on the symptoms or risk factors rather than the causes. More precisely, treatment of chorioamnionitis aims to induce or augment labor with adequate postnatal support. However, intrapartum therapy with intravenous antibiotics has shown to decrease neonatal sepsis and subsequent adverse outcomes. ${ }^{61}$ Moreover, amnion infusion with antibiotics has been used to treat established chorioamnionitis. ${ }^{61}$ Postulated mechanisms are dilution of infective organisms and antimicrobial effects of the fluid infused. ${ }^{61}$ Although amnion infusion with antibiotic treatment might protect against adverse fetal outcomes, including neurodevelopmental outcomes, this therapy has been aimed at eradication of bacteria, whereas other microorganisms (i.e. fungi and viruses) are not be affected by antibiotic treatment. Candida albicans ( $C$. Albicans) has frequently been isolated from amniotic fluid of women with preterm labor, and intraamniotic C. Albicans can cause fetal death or fetal candidiasis with impaired neurodevelopmental outcomes. ${ }^{62-64}$

Recently, in two clinical cases of intra-amniotic C. Albicans infection, treatment with intra-amniotic injections of Fluconazole, supplemented with oral and vaginal treatment resulted in prolonged pregnancy and survival of both neonates. ${ }^{65}$ Moreover, animal 
studies have demonstrated that intra-amniotic Fluconazole treatment reduced the fetal systemic inflammatory response elicited by intra-amniotic C. Albicans. ${ }^{62,66}$

Currently hypothermia is the only established neuroprotective intervention for hypoxiaischemia-induced injury of the term or late-preterm neonate, with modest protective effects if started within 6 hours after the insult. Preterm infants are excluded from this therapy due to an increased risk of adverse effects, illustrating a great demand for novel therapeutic interventions for preterm brain injury. ${ }^{67}$

\section{BONE MARROW-DERIVED PROGENITOR CELLS}

Cell-based interventions as a therapeutic strategy for injury to the (neonatal) central nervous system have gathered much attention during the past decade. ${ }^{68-76}$ Many different types of stem cells, derived from fetal, placental, and adult tissues are currently under investigation. ${ }^{77-82}$ Mesenchymal stromal cells (MSCs) are a subset of progenitors that have been shown to differentiate into multiple lineages (i.e. osteoblasts, adipocytes and chondrocytes). ${ }^{77-80}$ They have been the focus of research as they are easily obtainable (i.e. from cord blood, Wharton's jelly, bone marrow) and do not have the ethical and safety concerns of embryonic stem cells. ${ }^{73,76}$ Moreover, MSCs have low immunogenicity due to a lack of expression of MHC class II antigens, allowing the use for allogenic therapy. ${ }^{76}$ The therapeutic potential of MSCs is mainly attributed to their immune-modulatory and regenerative potential. ${ }^{73,74,79,83}$ The effects of MSCs on the immune system comprise both modulation of innate and adaptive immune responses from a pro-inflammatory status towards and anti-inflammatory status, thereby reducing tissue injury and creating an environment in favor of tissue repair and regeneration. $^{79,80,84}$

Besides immune modulation in favor of regeneration, MSCs directly affect the injured central nervous system through secretion of neurotrophic factors that stimulate and maintain neurogenesis of the endogenous neuronal stem cell population and subsequent differentiation into neuronal and oligodendroglial lineage. ${ }^{74,79,83,85-87}$ Recently, the neuroprotective potential of systemically administered MSCs was shown in a preclinical ovine model of global hypoxia-ischemia-induced injury of the preterm brain. ${ }^{88} \mathrm{MSC}$ administration in the therapeutic window of opportunity established functional improvement and prevented hypomyelination of the subcortical white matter. These protective effects were attributed to anti-inflammatory effects since neuroinflammation and peripheral immune activation were significantly reduced. ${ }^{88}$

However, clinical application of mesenchymal stromal cells requires large cell numbers within the limited time-frame of the window of opportunity. These criteria cannot be 
met by autologous transplantation and mandate an off-the-shelf MSC product for allogeneic transplantation.

Recently, multipotent adult progenitor cells (MAPCs), a subpopulation bone marrow derived stromal cells, have been described and characterized. Compared to MSCs, MAPCs have a more pronounced expansion potential, with stable genetics. In addition, MAPCs express lower levels of MHC class I molecules compared to MSCs. ${ }^{89-91}$ Similar to MSCs, MAPCs have immune-modulatory potential. ${ }^{90,} 91$ Moreover, a major obstacle for intravenous administration of cell-based therapies remains pulmonary passage. Fischer et al. demonstrated that due to their smaller size twice as many MAPCs bypass the pulmonary capillary bed compared to larger MSCs after intravenous injection. ${ }^{92}$

Pre-clinical animal studies clearly have demonstrated therapeutic benefits of MAPCs after traumatic brain injury and spinal cord injury. Moreover, in a murine model of ischemic stroke, intracranial injections of MAPC or MSCs revealed that MAPC had stronger effects on the attenuation of the inflammatory response and more potency to promote endogenous tissue regeneration than MSCs. ${ }^{93}$

Because of their extensive proliferation potential with stable genetics, low immunogenicity, and therapeutic efficacy, MAPCs are excellent candidates for large scale production and "off the shelf" administration. ${ }^{94}$ Off-the-shelf availability would be off great value with respect to the window of opportunity for treatment in that it makes cell-harvesting for allogeneic transplantation redundant.

The therapeutic potential of both MSCs and MAPCs largely has been attributed to release of paracrine mediators, stored in extracellular vesicles (i.e. exosomes (70-150 $\mathrm{nm}$ in diameter) and microvesicles (100-1000 nm)), rather than homing of cells and subsequent substitution of lost cell types. ${ }^{95}$ This paracrine hypothesis is confirmed by the fact that transplanted cells were trapped within the lungs and minor fractions could be detected in the brain following systemic administration. ${ }^{88,96,97}$

Exosomes are small membrane vesicles that arise from multivesicular endosomes and are released into the environment upon fusion with the plasma membrane. ${ }^{98}$ They contain coding and noncoding RNAs, lipids, and proteins. Originally thought to assist in trafficking of extracellular molecules to lysosomes for degradation, it is now clear that they play active roles in intercellular communication. ${ }^{99,} 100$ Mesenchymal stromal cellderived extracellular vesicles (MSC-EVs) have been shown to elicit similar biological effects to MSCs after administration in various preclinical disease models, including models for kidney, cardiac and brain injury. ${ }^{101-106}$ Moreover, recently, a therapyrefractory graft-versus-host disease patient was successfully treated with allogeneic MSC-EVs. MSC-EVs induced immune suppressive effects in vitro and in vivo without any other side effects. ${ }^{107}$ In addition, application of extracellular vesicles avoids potential concerns associated with administration of living cells such as tumor formation. ${ }^{106,108}$ 


\section{ANIMAL MODEL}

To study the effects of intra-uterine inflammation and hypoxia-ischemia on fetal brain injury and development, various animal models have been developed including mice, rats, guinea pigs, rabbits, pigs, and sheep. ${ }^{25}$ In this thesis, therapeutic strategies for preterm brain injury were investigated in the ovine fetus, since ovine developmental biology closely resembles the human situation. For example, alveolarization of the fetal lungs, formation of intestinal crypts and villi, and white matter myelination predominantly occur in utero in both humans and sheep, whereas these processes are postnatal events in other species (i.e. rodents). ${ }^{109}$ Furthermore, as in humans, sheep have a gyrencephalic brain compared to rodents that are lisencephalic and the abundance of cerebral white matter and its anatomic similarities to that of the preterm infant make fetal sheep ideal for neuropathological correlation with humans. ${ }^{110}$ The long gestation of fetal sheep ( $\sim 147$ days) allows selection of an appropriate developmental stage during which brain insults can be induced and evaluated. Additionally, the docile nature of sheep allows for minimal invasive handling for induction of inflammation through intra-amniotic injections of micro-organisms or endotoxins. Also, the relative large size of fetal lambs allows for chronic instrumentation to enable physiological measurements (e.g. blood pressure, oxygenation), electrophysiological recordings, and application of clinical relevant insults (i.e. intraamniotic injection of infectious agents and global hypoxia-ischemia. Therefore, preterm sheep are considered to be an excellent translational model to study the effects of inflammation on the developing brain.

\section{THE INSTRUMENTED FETAL SHEEP MODEL}

All studies comprising global hypoxia-ischemia in this thesis were performed in a translational model of global hypoxia-ischemia in the preterm ovine fetus in which ovine fetuses were equipped with vascular catheters, electrocardiogram and electro encephalogram electrodes and an inflatable vascular occluder around the umbilical cord

for remote induction of transient umbilical cord occlusion in utero (figure 1). ${ }^{45,88,111}$ Chronic instrumentation allows for continuous recordings and off-line analysis of vital physiological parameters such as brain function with EEG, electrophysiological cardiac function with ECG, hemodynamic stability (baroreflex) with blood pressure and ECG, and blood gas analysis. 


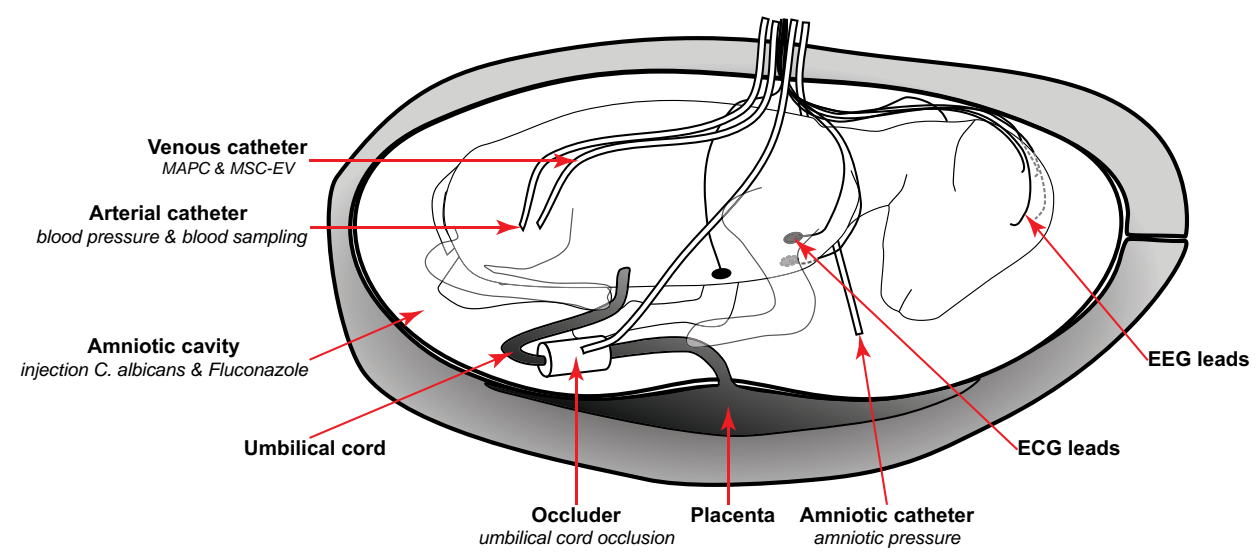

Figure 1. Sheep model of fetal brain injury caused by hypoxia-ischemia or intra-uterine infection.

For experiments comprising global hypoxia-ischemia (i.e. Chapters 2 and 3), ovine fetuses were equipped with electroencephalogram electrodes on the head and electrocardiogram electrodes and thorax. An inflatable vascular occluder was placed around the umbilical for induction of global hypoxia-ischemia. An arterial catheter was placed for blood pressure monitoring and blood sampling. A venous catheter was placed for administration of MAPC cells or MSC-EVs. An additional amniotic catheter was placed for measurement of amniotic pressure and subtracted from the arterial blood pressure to obtain the real blood pressure.

In chapter 4, intra-uterine infection was induced by injecting C. albicans directly into the amniotic cavity under ultrasound guidance without surgical intervention and instrumentation. Fluconazole was injected into the amniotic cavity under ultrasound guidance.

\section{OUTLINE OF THE THESIS}

The aim of the current thesis was to investigate therapeutic strategies for the prevention of injury of the preterm brain caused by infectious and sterile inflammatory triggers.

In chapters 2 and 3 neuroprotective strategies for sterile inflammation caused by global hypoxia-ischemia are described. Cell-based therapies have proven neuroprotective potential following global hypoxia-ischemia. A major obstacle, however, is the availability of sufficient numbers of stem cells within the limited time in the "window of opportunity". In chapter 2 we investigated the neuroprotective potential of systemically delivered clinical-grade multipotent adult progenitor cells after global hypoxia-ischemia in the preterm ovine fetus. We hypothesized that systemic administration of a clinicalgrade MAPCs product, would attenuate cerebral and peripheral inflammation and prevent structural and functional brain injury after global $\mathrm{HI}$ in the preterm ovine fetus.

The promising potential for cell-based therapies as a treatment for preterm brain injury are considered to be primarily driven by paracrine mediators, stored in extracellular vesicles. This suggests that administration of extracellular vesicles rather than intact 
stem cells are sufficient to exert therapeutic effects. In chapter 3, we therefore investigated the protective effects of systemically administered mesenchymal stromal cell-derived extracellular vesicles (MSC-EVs) after hypoxia-ischemia-induced injury of the preterm ovine brain. We hypothesized that systemic administration of MSC-EVs would be neuroprotective in hypoxic-ischemic injury in the preterm brain.

In chapter 4, we studied the mechanisms underlying C. albicans-induced fetal brain injury. We hypothesized that antenatal exposure to $C$. albicans caused a neuroinflammatory response and subsequent white matter injury, which we tested by exposing fetal sheep to intra-amniotic $C$. albicans. In addition, we tested whether these potential adverse outcomes were improved by in utero antifungal treatment with Fluconazole. We therefore further hypothesized that fetal intra-amniotic and intraperitoneal administration of Fluconazole would reduce the neuroinflammatory response and subsequent white matter injury to the fetal brain. 


\section{REFERENCES}

1. Goldenberg, R.L., Culhane, J.F., lams, J.D. \& Romero, R. Epidemiology and causes of preterm birth. Lancet 371, 75-84 (2008).

2. EUROCAT., E.-P.P.w.S.a. European Perinatal Health Report. The health and care of pregnant women and babies in Europe in 2010.; 2013.

3. Saigal, S. \& Doyle, L.W. An overview of mortality and sequelae of preterm birth from infancy to adulthood. The Lancet 371, 261-269 (2008).

4. Doyle, L.W. et al. Bronchopulmonary dysplasia in very low birth weight subjects and lung function in late adolescence. Pediatrics 118, 108-113 (2006).

5. Gantert, M. et al. Chorioamnionitis: a multiorgan disease of the fetus? J Perinatol 30 Suppl, S21-30 (2010).

6. Volpe, J.J. Brain injury in premature infants: a complex amalgam of destructive and developmental disturbances. The Lancet Neurology 8, 110-124 (2009).

7. Dubois, J. et al. The early development of brain white matter: a review of imaging studies in fetuses, newborns and infants. Neuroscience 276, 48-71 (2014).

8. Back, S.A., Riddle, A. \& McClure, M.M. Maturation-dependent vulnerability of perinatal white matter in premature birth. Stroke 38, 724-730 (2007).

9. Section II Chapter 6: Biological pathways leading to preterm birth. In: Behrman, R.E. \& Stith Butler, A. (eds). Preterm birth: causes, consequences and prevention. National Academies Press: Washington, 2007, pp 169-206.

10. Goldenberg, R.L., Hauth, J.C. \& Andrews, W.W. Intrauterine infection and preterm delivery. N Eng/ J Med 342, 1500-1507 (2000).

11. Section II Chapter 5: Medical and pregnancy conditions associated with preterm birth. In: Behrman, R.E. \& Stith Butler, A. (eds). Preterm birth: causes, consequences and prevention. National Academies Press: Washington, 2007, pp 148-168.

12. Payne, M.S. \& Bayatibojakhi, S. Exploring preterm birth as a polymicrobial disease: an overview of the uterine microbiome. Frontiers in immunology 5 (2014).

13. Perni, S.C. et al. Mycoplasma hominis and Ureaplasma urealyticum in midtrimester amniotic fluid: association with amniotic fluid cytokine levels and pregnancy outcome. Am J Obstet Gynecol 191, 13821386 (2004).

14. Steel, J.H. et al. Bacteria and inflammatory cells in fetal membranes do not always cause preterm labor. Pediatr Res 57, 404-411 (2005).

15. Iwasaka, T., Wada, T., Kidera, Y. \& Sugimori, H. Hormonal status and mycoplasma colonization in the female genital tract. Obstet Gynecol 68, 263-266 (1986).

16. Elovitz, M.A. et al. Intrauterine inflammation, insufficient to induce parturition, still evokes fetal and neonatal brain injury. International Journal of Developmental Neuroscience 29, 663-671 (2011).

17. Yoon, B.H. et al. The relationship among inflammatory lesions of the umbilical cord (funisitis), umbilical cord plasma interleukin 6 concentration, amniotic fluid infection, and neonatal sepsis. American journal of obstetrics and gynecology 183, 1124-1129 (2000).

18. Mueller-Heubach, E., Rubinstein, D.N. \& Schwarz, S.S. Histologic chorioamnionitis and preterm delivery in different patient populations. Obstetrics and gynecology 75, 622-626 (1990).

19. Russell, P. Inflammatory lesions of the human placenta. III. The histopathology of villitis of unknown aetiology. Placenta 1, 227-244 (1980).

20. Romero, R. et al. Inflammation in preterm and term labour and delivery. Seminars in Fetal and Neonatal Medicine; 2006: Elsevier; 2006. p. 317-326.

21. DiGiulio, D.B. et al. Prevalence and diversity of microbes in the amniotic fluid, the fetal inflammatory response, and pregnancy outcome in women with preterm pre-labor rupture of membranes. Am J Reprod Immunol 64, 38-57 (2010). 
22. DiGiulio, D.B. et al. Microbial prevalence, diversity and abundance in amniotic fluid during preterm labor: a molecular and culture-based investigation. PLoS One 3, e3056 (2008).

23. Hillier, S.L. et al. A case-control study of chorioamnionic infection and histologic chorioamnionitis in prematurity. N Engl J Med 319, 972-978 (1988).

24. Gotsch, F. et al. The fetal inflammatory response syndrome. Clin Obstet Gynecol 50, 652-683 (2007).

25. Kuypers, E. et al. White matter injury following fetal inflammatory response syndrome induced by chorioamnionitis and fetal sepsis: lessons from experimental ovine models. Early human development 88, 931-936 (2012).

26. Degos, V. et al. Inflammation processes in perinatal brain damage. Journal of neural transmission 117, 1009-1017 (2010).

27. Hagberg, H., Gressens, P. \& Mallard, C. Inflammation during fetal and neonatal life: implications for neurologic and neuropsychiatric disease in children and adults. Annals of neurology 71, 444-457 (2012).

28. Malaeb, S. \& Dammann, O. Fetal inflammatory response and brain injury in the preterm newborn. Journal of child neurology (2009).

29. Liu, X.-B., Shen, Y., Plane, J.M. \& Deng, W. Vulnerability of premyelinating oligodendrocytes to whitematter damage in neonatal brain injury. Neuroscience bulletin 29, 229-238 (2013).

30. Drury, P.P., Bennet, L. \& Gunn, A.J. Mechanisms of hypothermic neuroprotection. Seminars in Fetal and Neonatal Medicine; 2010: Elsevier; 2010. p. 287-292.

31. Yenari, M.A. \& Han, H.S. Neuroprotective mechanisms of hypothermia in brain ischaemia. Nature Reviews Neuroscience 13, 267-278 (2012).

32. Volpe, J.J. Perinatal brain injury: from pathogenesis to neuroprotection. Mental retardation and developmental disabilities research reviews 7, 56-64 (2001).

33. Roelfsema, V. et al. Window of opportunity of cerebral hypothermia for postischemic white matter injury in the near-term fetal sheep. Journal of Cerebral Blood Flow \& Metabolism 24, 877-886 (2004).

34. Bianchi, M.E. DAMPs, PAMPs and alarmins: all we need to know about danger. Journal of leukocyte biology 81, 1-5 (2007).

35. Khwaja, O. \& Volpe, J. Pathogenesis of cerebral white matter injury of prematurity. Archives of Disease in Childhood-Fetal and Neonatal Edition 93, F153-F161 (2008).

36. Baburamani, A.A., Supramaniam, V.G., Hagberg, H. \& Mallard, C. Microglia toxicity in preterm brain injury. Reproductive Toxicology 48, 106-112 (2014).

37. Hagberg, H. et al. The role of inflammation in perinatal brain injury. Nature Reviews Neurology 11, 192208 (2015).

38. Czeh, M., Gressens, P. \& Kaindl, A.M. The yin and yang of microglia. Developmental neuroscience 33, 199-209 (2011).

39. Hanisch, U.-K. \& Kettenmann, H. Microglia: active sensor and versatile effector cells in the normal and pathologic brain. Nature neuroscience 10, 1387-1394 (2007).

40. Almolda, B., González, B. \& Castellano, B. Are microglial cells the regulators of lymphocyte responses in the CNS? Frontiers in cellular neuroscience 9 (2015).

41. Alvarez-Diaz, A., Hilario, E., Goni de Cerio, F., Valls-i-Soler, A. \& Alvarez-Diaz, F. Hypoxic-ischemic injury in the immature brain-key vascular and cellular players. Neonatology 92, 227-235 (2007).

42. Daré, E., Schulte, G., Karovic, O., Hammarberg, C. \& Fredholm, B.B. Modulation of glial cell functions by adenosine receptors. Physiology \& behavior 92, 15-20 (2007).

43. Gelderblom, M. et al. Temporal and spatial dynamics of cerebral immune cell accumulation in stroke. Stroke 40, 1849-1857 (2009).

44. Fathali, N. et al. Splenic immune cells in experimental neonatal hypoxia-ischemia. Translational stroke research 4, 208-219 (2013).

45. Jellema, R.K. et al. Cerebral inflammation and mobilization of the peripheral immune system following global hypoxia-ischemia in preterm sheep. J Neuroinflammation 10, 2094-2010 (2013).

46. Back, S.A. et al. Late oligodendrocyte progenitors coincide with the developmental window of vulnerability for human perinatal white matter injury. The Journal of Neuroscience 21, 1302-1312 (2001). 
47. Back, S.A., Luo, N.L., Borenstein, N.S., Volpe, J.J. \& Kinney, H.C. Arrested oligodendrocyte lineage progression during human cerebral white matter development: dissociation between the timing of progenitor differentiation and myelinogenesis. Journal of Neuropathology \& Experimental Neurology 61, 197-211 (2002).

48. Volpe, J.J., Kinney, H.C., Jensen, F.E. \& Rosenberg, P.A. The developing oligodendrocyte: key cellular target in brain injury in the premature infant. International Journal of Developmental Neuroscience 29, 423-440 (2011).

49. Gunn, A.J. \& Bennet, L. Fetal hypoxia insults and patterns of brain injury: insights from animal models. Clinics in perinatology 36, 579-593 (2009).

50. McQuillen, P.S., Sheldon, R.A., Shatz, C.J. \& Ferriero, D.M. Selective vulnerability of subplate neurons after early neonatal hypoxia-ischemia. The Journal of neuroscience 23, 3308-3315 (2003).

51. McQuillen, P. \& Ferriero, D. Perinatal subplate neuron injury: implications for cortical development and plasticity. Brain pathology 15, 250-260 (2005).

52. Toet, M., Hellström-Westas, L., Groenendaal, F., Eken, P. \& De Vries, L. Amplitude integrated EEG 3 and 6 hours after birth in full term neonates with hypoxic-ischaemic encephalopathy. Archives of Disease in Childhood-Fetal and Neonatal Edition 81, F19-F23 (1999).

53. Glass, H.C. et al. Clinical neonatal seizures are independently associated with outcome in infants at risk for hypoxic-ischemic brain injury. The Journal of pediatrics 155, 318-323 (2009).

54. Miller, S. et al. Seizure-associated brain injury in term newborns with perinatal asphyxia. Neurology 58, 542-548 (2002).

55. El-Dib, M., Chang, T., Tsuchida, T.N. \& Clancy, R.R. Amplitude-integrated electroencephalography in neonates. Pediatric neurology 41, 315-326 (2009).

56. Hellström-Westas, L. \& Rosén, I. Continuous brain-function monitoring: state of the art in clinical practice. Seminars in Fetal and Neonatal Medicine; 2006: Elsevier; 2006. p. 503-511.

57. Niemarkt, H.J. et al. Multi-channel amplitude-integrated EEG characteristics in preterm infants with a normal neurodevelopment at two years of corrected age. Early human development 88, 209-216 (2012).

58. Clancy, R.R. et al. Agreement between long-term neonatal background classification by conventional and amplitude-integrated EEG. Journal of Clinical Neurophysiology 28, 1-9 (2011).

59. Zwanenburg, A. et al. Heart rate-mediated blood pressure control in preterm fetal sheep under normal and hypoxic-ischemic conditions. Pediatr Res 73, 420-426 (2013).

60. Perlman, J.M. The relationship between systemic hemodynamic perturbations and periventricularintraventricular hemorrhage-A historical perspective. Seminars in pediatric neurology; 2009: Elsevier; 2009. p. 191-199.

61. Kiiza, J.A. \& Hofmeyr, G.J. Amnioinfusion for chorioamnionitis. The Cochrane Library (2015).

62. Maneenil, G. et al. Fluconazole treatment of intrauterine Candida albicans infection in fetal sheep. Pediatr Res 77, 740-748 (2015).

63. Benjamin, D.K. et al. Neonatal candidiasis among extremely low birth weight infants: risk factors, mortality rates, and neurodevelopmental outcomes at 18 to 22 months. Pediatrics 117, 84-92 (2006).

64. Darmstadt, G.L., Dinulos, J.G. \& Miller, Z. Congenital cutaneous candidiasis: clinical presentation, pathogenesis, and management guidelines. Pediatrics 105, 438-444 (2000).

65. Bean, L.M., Jackson, J.R., Dobak, W.J., Beiswenger, T.R. \& Thorp, J.A. Intra-amniotic fluconazole therapy for Candida albicans intra-amniotic infection. Obstetrics \& Gynecology 121, 452-454 (2013).

66. Payne, M.S. et al. Intrauterine Candida albicans infection elicits severe inflammation in fetal sheep. Pediatr Res 75, 716-722 (2014).

67. Jacobs, S.E., Hunt, R., Tarnow-Mordi, W.O., Inder, T.E. \& Davis, P.G. Cochrane Review: Cooling for newborns with hypoxic ischaemic encephalopathy. Evidence-Based Child Health: A Cochrane Review Journal 3, 1049-1115 (2008).

68. Bennet, L. et al. Cell therapy for neonatal hypoxia-ischemia and cerebral palsy. Annals of neurology 71, 589-600 (2012).

69. Titomanlio, L. et al. Stem cell therapy for neonatal brain injury: perspectives and challenges. Annals of neurology 70, 698-712 (2011). 


\section{CHAPTER 1}

70. van Velthoven, C.T., Kavelaars, A., van Bel, F. \& Heijnen, C.J. Regeneration of the ischemic brain by engineered stem cells: fuelling endogenous repair processes. Brain research reviews 61, 1-13 (2009).

71. Gortner, L. et al. Regenerative therapies in neonatology: clinical perspectives. Klinische Pädiatrie 224, 233 (2012).

72. Borlongan, C.V. \& Weiss, M.D. Baby STEPS: a giant leap for cell therapy in neonatal brain injury. Pediatr Res 70, 3-9 (2011).

73. Donega, V., van Velthoven, C.T., Nijboer, C.H., Kavelaars, A. \& Heijnen, C.J. The endogenous regenerative capacity of the damaged newborn brain: boosting neurogenesis with mesenchymal stem cell treatment. Journal of Cerebral Blood Flow \& Metabolism 33, 625-634 (2013).

74. van Velthoven, C.T., Kavelaars, A. \& Heijnen, C.J. Mesenchymal stem cells as a treatment for neonatal ischemic brain damage. Pediatr Res 71, 474-481 (2012).

75. Dalous, J. et al. Use of human umbilical cord blood mononuclear cells to prevent perinatal brain injury: a preclinical study. Stem cells and development 22, 169-179 (2012).

76. Fleiss, B. et al. Stem cell therapy for neonatal brain injury. Clinics in perinatology 41, 133-148 (2014).

77. Jiang, Y. et al. Pluripotency of mesenchymal stem cells derived from adult marrow. Nature 418, 41-49 (2002).

78. Méndez-Ferrer, S. et al. Mesenchymal and haematopoietic stem cells form a unique bone marrow niche. nature 466, 829-834 (2010).

79. Uccelli, A., Moretta, L. \& Pistoia, V. Mesenchymal stem cells in health and disease. Nature Reviews Immunology 8, 726-736 (2008).

80. Le Blanc, K. \& Mougiakakos, D. Multipotent mesenchymal stromal cells and the innate immune system. Nature Reviews Immunology 12, 383-396 (2012).

81. Bieback, K., Schallmoser, K., Klüter, H. \& Strunk, D. Clinical protocols for the isolation and expansion of mesenchymal stromal cells. Transfusion Medicine and Hemotherapy 35, 286-294 (2008).

82. Portmann-Lanz, C.B. et al. Placental mesenchymal stem cells as potential autologous graft for pre-and perinatal neuroregeneration. American journal of obstetrics and gynecology 194, 664-673 (2006).

83. Uccelli, A., Benvenuto, F., Laroni, A. \& Giunti, D. Neuroprotective features of mesenchymal stem cells. Best practice \& research Clinical haematology 24, 59-64 (2011).

84. Siegel, G., Schäfer, R. \& Dazzi, F. The immunosuppressive properties of mesenchymal stem cells. Transplantation 87, S45-S49 (2009).

85. van Velthoven, C.T., Kavelaars, A., van Bel, F. \& Heijnen, C.J. Repeated mesenchymal stem cell treatment after neonatal hypoxia-ischemia has distinct effects on formation and maturation of new neurons and oligodendrocytes leading to restoration of damage, corticospinal motor tract activity, and sensorimotor function. The Journal of Neuroscience 30, 9603-9611 (2010).

86. Rivera, F.J. et al. Mesenchymal stem cells instruct oligodendrogenic fate decision on adult neural stem cells. Stem Cells 24, 2209-2219 (2006).

87. Steffenhagen, C. et al. Mesenchymal stem cells prime proliferating adult neural progenitors toward an oligodendrocyte fate. Stem cells and development 21, 1838-1851 (2011).

88. Jellema, R.K. et al. Mesenchymal stem cells induce T-cell tolerance and protect the preterm brain after global hypoxia-ischemia. (2013).

89. Jacobs, S.A., Roobrouck, V.D., Verfaillie, C.M. \& Van Gool, S.W. Immunological characteristics of human mesenchymal stem cells and multipotent adult progenitor cells. Immunol Cell Biol 91, 32-39 (2013).

90. Jacobs, S.A. et al. Mutual interaction between Human Multipotent Adult Progenitor Cells and NK cells. Cell Transplant (2013).

91. Jacobs, S.A. et al. Human multipotent adult progenitor cells are nonimmunogenic and exert potent immunomodulatory effects on alloreactive T-cell responses. Cell Transplant 22, 1915-1928 (2013).

92. Fischer, U.M. et al. Pulmonary passage is a major obstacle for intravenous stem cell delivery: the pulmonary first-pass effect. Stem cells and development 18, 683-692 (2009).

93. Mora-Lee, S. et al. Therapeutic effects of hMAPC and hMSC transplantation after stroke in mice. PLoS One 7, e43683 (2012). 
94. Vaes, B., Van't Hof, W., Deans, R. \& Pinxteren, J. Application of MultiStem ${ }^{\circledR}$ allogeneic cells for immunomodulatory therapy: clinical progress and pre-clinical challenges in prophylaxis for graft versus host disease. Frontiers in immunology 3 (2012).

95. Lai, R.C., Chen, T.S. \& Lim, S.K. Mesenchymal stem cell exosome: a novel stem cell-based therapy for cardiovascular disease. Regenerative medicine 6, 481-492 (2011).

96. Lee, R.H. et al. Intravenous hMSCs improve myocardial infarction in mice because cells embolized in lung are activated to secrete the anti-inflammatory protein TSG-6. Cell stem cell 5, 54-63 (2009).

97. van Velthoven, C.T., Kavelaars, A., van Bel, F. \& Heijnen, C.J. Mesenchymal stem cell transplantation changes the gene expression profile of the neonatal ischemic brain. Brain, behavior, and immunity 25, 1342-1348 (2011).

98. Théry, C., Zitvogel, L. \& Amigorena, S. Exosomes: composition, biogenesis and function. Nature Reviews Immunology 2, 569-579 (2002).

99. Raposo, G. et al. B lymphocytes secrete antigen-presenting vesicles. The Journal of experimental medicine 183, 1161-1172 (1996).

100. Zitvogel, L. et al. Eradication of established murine tumors using a novel cell-free vaccine: dendritic cell derived exosomes. Nature medicine 4, 594-600 (1998).

101. Lai, R.C. et al. Exosome secreted by MSC reduces myocardial ischemia/reperfusion injury. Stem cell research 4, 214-222 (2010).

102. Bruno, S. et al. Mesenchymal stem cell-derived microvesicles protect against acute tubular injury. Journal of the American Society of Nephrology 20, 1053-1067 (2009).

103. Xin, H. et al. Systemic administration of exosomes released from mesenchymal stromal cells promote functional recovery and neurovascular plasticity after stroke in rats. Journal of Cerebral Blood Flow \& Metabolism 33, 1711-1715 (2013).

104. Gatti, S. et al. Microvesicles derived from human adult mesenchymal stem cells protect against ischaemia-reperfusion-induced acute and chronic kidney injury. Nephrology Dialysis Transplantation, gfr015 (2011).

105. Bruno, S. et al. Microvesicles derived from mesenchymal stem cells enhance survival in a lethal model of acute kidney injury. (2012).

106. Doeppner, T.R. et al. Extracellular Vesicles Improve Post-Stroke Neuroregeneration and Prevent Postischemic Immunosuppression. Stem cells translational medicine 4, 1131-1143 (2015).

107. Kordelas, L. et al. MSC-derived exosomes: a novel tool to treat therapy-refractory graft-versus-host disease. Leukemia 28, 970-973 (2014).

108. Dlouhy, B.J., Awe, O., Rao, R.C., Kirby, P.A. \& Hitchon, P.W. Autograft-derived spinal cord mass following olfactory mucosal cell transplantation in a spinal cord injury patient: Case report. Journal of Neurosurgery: Spine 21, 618-622 (2014).

109. Wolfs, T.G. et al. Inflammation-induced immune suppression of the fetus: a potential link between chorioamnionitis and postnatal early onset sepsis. The Journal of Maternal-Fetal \& Neonatal Medicine 25, 8-11 (2012).

110. Back, S.A., Riddle, A., Dean, J. \& Hohimer, A.R. The instrumented fetal sheep as a model of cerebral white matter injury in the premature infant. Neurotherapeutics 9, 359-370 (2012).

111. Jellema, R.K. et al. Systemic G-CSF attenuates cerebral inflammation and hypomyelination but does not reduce seizure burden in preterm sheep exposed to global hypoxia-ischemia. Experimental neurology 250, 293-303 (2013). 



\section{Clinical-grade Multipotent Adult Progenitor Cells for Hypoxic-Ischemic Injury in the Preterm Brain}

Based on:

Clinical-grade Multipotent Adult Progenitor Cells for Hypoxic-Ischemic Injury in the Preterm Brain.

Reint K. Jellema*, Daan R.M.G. Ophelders", Alex Zwanenburg, Maria Nikiforou, Tammo Delhaas, Peter Andriessen, Robert mays, Robert Deans, Wilfred Germeraad, Tim G.A.M. Wolfs, Boris W. Kramer.

Journal of Neuroinflammation. 2015;12(1):241.

* Contributed equally 


\section{ABSTRACT}

Preterm infants are at risk for hypoxic-ischemic encephalopathy. No therapy exists to treat this brain injury and subsequent long-term sequelae. We have previously shown in a well-established preclinical model of global hypoxia-ischemia (HI) that mesenchymal stromal cells are a promising candidate for the treatment of hypoxic-ischemic brain injury. In the current study we investigated the neuroprotective capacity of multipotent adult progenitor cells (MAPC), which are adherent bone marrow derived cells of an earlier developmental stage than mesenchymal stromal cells and exhibiting more potent anti-inflammatory and regenerative properties.

Instrumented preterm sheep fetuses were subjected to global hypoxia-ischemia by 25 minutes of umbilical cord occlusion at a gestational age of 106 (term 147) days. During a 7-day reperfusion period, vital parameters (e.g. blood pressure and heart rate; baroreceptor reflex) and (amplitude-integrated) electroencephalogram were recorded. At the end of the experiment, the preterm brain was studied by histology.

Systemic administration of MAPC reduced the number and duration of seizures and prevented decrease in baroreceptor reflex sensitivity after global HI. In addition, MAPC prevented $\mathrm{HI}$-induced microglial proliferation in the preterm brain. These antiinflammatory effects were associated with MAPC-induced prevention of hypomyelination after global $\mathrm{HI}$. Besides attenuation of the cerebral inflammatory response, our findings showed that MAPC modulated the peripheral splenic inflammatory response, which has been implicated in the etiology of hypoxic-ischemic injury in the preterm brain.

In a preclinical animal model MAPCs improved the functional and structural outcome of the preterm brain after global HI. Future studies should establish the mechanism and long-term therapeutic effects of neuroprotection established by MAPCs in the developing preterm brain exposed to HI. Our study may form the basis for future clinical trials, which will evaluate whether MAPCS therapy is capable of reducing neurological sequelae in preterm infants with hypoxic-ischemic encephalopathy. 


\section{INTRODUCTION}

Hypoxia-ischemia (HI) in the developing brain is strongly correlated with mortality and neurological morbidity in preterm and full-term infants, resulting in an enormous physical, psychological and economic burden. ${ }^{1}$ Unfortunately, no therapeutic cure is available for hypoxic-ischemic brain injury in preterm infants.

In a translational animal model of global $\mathrm{HI}$ in the preterm ovine fetus, our group has demonstrated that cell-based therapy may be a promising neuroprotective strategy for preterm neonates suffering from $\mathrm{HI}$-induced brain injury. ${ }^{2}$ We showed that intravenous administration of exogenous mesenchymal stromal cells (MSCs) protected against functional loss and structural injury of the preterm brain. ${ }^{2}$ These neuroprotective effects were largely attributable to attenuation of (neuro) inflammatory processes, as indicated by decreased microglial activation and proliferation in the preterm brain and induction of peripheral T-cell tolerance, which was associated with reduced cerebral infiltration of these immune effector cells. ${ }^{2}$

Multipotent adult progenitor cells (MAPCs), which are adherent bone marrow derived cells of an earlier developmental stage than MSCs have a high expansion potential and their immunological properties make it possible to use them as a universal allogeneic donor. ${ }^{3-5}$ Pre-clinical animal studies have demonstrated that in comparison to MSCs, MAPCs have stronger anti-inflammatory effects and are more potent in promoting endogenous tissue regeneration after ischemic and traumatic injury to the central nervous system (CNS). ${ }^{6-11}$ In addition, the smaller size of MAPCs compared to MSCs facilitates passage through the pulmonary capillary bed, which may increase availability of MAPCs in the systemic and cerebral vasculature and thus enhancing their neuroprotective effect. ${ }^{12}$

Based on these superior qualities of MAPCs, we aimed to assess the anti-inflammatory and neuroprotective potential of MAPCs in the preterm brain exposed to global HI. We hypothesized that systemic administration of a clinical-grade MAPC product, would attenuate cerebral and peripheral inflammation and prevent structural and functional brain injury after global $\mathrm{HI}$ in the preterm ovine fetus. We tested our hypothesis in a well-established pre-clinical animal model of preterm global HI. In this model, preterm ovine fetuses were exposed to global $\mathrm{HI}$ by transient umbilical cord occlusion (UCO) at 0.7 of gestation which is equivalent to 30-32 weeks human gestation ${ }^{13}$, followed by systemic administration of MAPCs during a 7 day reperfusion period.

The anti-inflammatory effects of MAPCs were assessed in the preterm brain by analysis of microglial responses in the hippocampus and subcortical white matter. The spleen was assessed, since previous reports suggest that MAPC-induced alterations in the splenic inflammatory response may be responsible for their neuroprotective effect after brain injury. ${ }^{9}$ Structural effects of systemic MAPC administration were assessed by histological examination of white matter injury. Brain function was studied by analysis 
of cortical function by means of electrographic seizure activity and central (brain stem) function by means of baroreceptor reflex sensitivity.

\section{MATERIALS \& METHODS}

\section{Study approval}

The experimental protocol and study design were in line with the institutional guides for animal experiments and were approved by the institutional Animal Ethics Research committee of Maastricht University, the Netherlands.

\section{Randomization and blinding}

Thirty-two singleton fetuses of time-mated Texel ewes were randomized by an independent researcher who was not involved in the animal experiments. Randomization resulted in four experimental groups: (1) sham umbilical cord occlusion, saline treatment (sham-SAL $n=8$ ), (2) sham umbilical cord occlusion, MAPC treatment (sham-MAPC; $n=8$ ), (3) umbilical cord occlusion, saline treatment (HI-SAL; $n=8$ ) and (4) umbilical cord occlusion, MAPC treatment (HI-MAPC; $n=8$ ) (Figure 1). The investigator performing the (sham) umbilical cord occlusions was blinded to treatment allocation. Tissue sampling and the analyses of brain tissue and electrophysiological data were conducted in a blinded fashion.

\section{Animals and surgery}

Singleton fetuses were surgically instrumented at 102 days gestational age (term 147 days) as described previously. ${ }^{14}$ Briefly, fetuses were partially exposed through a midline incision. A polyurethane umbilical vessel catheter $(1.2 \mathrm{~mm}$, Covidien, Mansfield, Massachusetts, USA) was placed in the left femoral artery and brachial vein for blood pressure recordings, blood sampling, and administration of MAPC. An additional catheter was placed into the amniotic sac for measurement of amniotic fluid pressure. Three electrocardiogram (ECG) electrodes were placed on the fetal thorax for cardiac monitoring. Two pairs of custom-made shielded silver-tipped electroencephalogram (EEG) electrodes (Cooner wire Co., Chatsworth, CA, USA) were placed bilaterally on the dura over the parasagittal parietal cortex ( 5 with a subcutaneous silver reference electrode placed in the neck. An inflatable vascular occluder (OC16HD, 16mm, In Vivo Metric, Healdsburg, CA, USA) was placed around the umbilical cord for induction of transient global HI. All fetal leads were exteriorized through the maternal flank. Post-operatively sheep were housed individually with access to water and food ad libitum. A period of 4 days post-operative recovery was incorporated before onset of the experiments. 


\section{Experimental design}

At 106 days gestational age (experimental day 0) fetuses were subjected to 25 minutes of (sham) umbilical cord occlusion by rapidly inflating the occluder with sterile saline of a defined volume known to completely inflate the occluder. An acute drop in heart rate and a gradual decline in blood pressure confirmed complete occlusion of the umbilical cord (Figure 2). Global hypoxia-ischemia was further monitored with subsequent arterial blood gas analysis indicating acidemia, hypoxemia and hypercapnia (data not shown). One hour after umbilical cord occlusion and on experimental day 4 fetuses received either an intravenous bolus of MAPCs or an equal volume of vehicle. At the end of the experiment (experimental day 7 ), both ewe and fetus were euthanized by administration of pentobarbital $(200 \mathrm{mg} / \mathrm{kg})$.

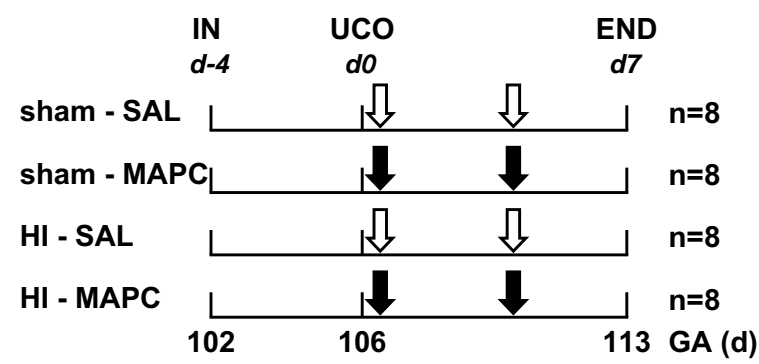

Figure 1. Study design.

Fetuses were instrumented at a gestational age (GA) of $102 \mathrm{~d}$. After a recovery period of $4 \mathrm{~d}$ fetuses were subjected to 25 min of umbilical cord occlusion (UCO) or sham. One hour and 4 days after UCO or sham, fetuses received either intravenous MAPCs (10 million cells, closed arrow) or saline 0.9\% (open arrow). After a $7 \mathrm{~d}$ reperfusion period brain tissue was collected. Abbreviations: in = instrumentation, $\mathrm{HI}=$ hypoxia-ischemia, $\mathrm{SAL}=$ saline, $\mathrm{MAPC}=$ multipotent adult progenitor cells.

\section{Data acquisition and analysis}

Physiological data was sampled as described previously on a custom-made MPAQ unit (Maastricht-Programmable AcQuisition system, Maastricht Instruments BV, Maastricht, the Netherlands) with IDEEQ software (Maastricht Instruments BV). ${ }^{14}$ In short, EEG data were sampled at $1000 \mathrm{~Hz}$ and stored on hard-disk for off-line analysis. EEG data were filtered using a $0.5-30 \mathrm{~Hz} 4^{\text {th }}$ order Butterworth band-pass filter. EEG signal with an amplitude $>1000 \mu \mathrm{V}$ was considered an artifact and removed from analysis $(<1 \%$ of data). After filtering, the raw EEG signals of the central and posterior channels were converted into amplitude-integrated EEG (aEEG) traces, using an (Matlab based) algorithm similar to the clinical EEG NicoletOne ${ }^{T M}$ device (Viasys Healthcare, Conshohocken, PA, USA), as previously described. 2, 15-18 EEG seizure activity was annotated manually using aEEG/EEG traces and the total number and length of seizures 
was subsequently calculated. A neonatologist experienced in aEEG interpretation and blinded to treatment allocation, performed annotation.

ECG and blood pressure data were processed off-line using MATLAB R2013a (The MathWorks, Inc., Natick, MA). The ECG recordings were used to determine R-R intervals. Blood pressure measurements were corrected for amniotic pressure and used to determine the mean arterial blood pressure in each pressure wave. As previously reported, heart rate and heart rate variability were assessed in $192 \mathrm{~s}$ segment by calculating the mean $\mathrm{R}-\mathrm{R}$ interval and standard deviation (SD-RR) respectively. ${ }^{19,} 20$ Likewise, mean systolic blood pressure and blood pressure variability was assessed by calculating the mean SBP and standard deviation (SD-SBP). ${ }^{19}$ As a time series estimate of baroreceptor reflex sensitivity, we calculated the ratio between SD-RR and SD-SBP. ${ }^{19,21,22}$

\section{MAPCS}

Multipotent Adult Progenitor Cells were provided by Athersys, Inc. (Cleveland OH, USA) and stored in liquid nitrogen. Cells were prepared under good manufacturing practice (GMP) and genomic and epigenetic stability of the cells were confirmed as previously described. ${ }^{5,23}$ Flow cytometric analysis of cell-surface proteins demonstrated that the cells were CD29, CD90, CD44 positive, and MHC class I low, and were negative for MHC class II, CD45, CD106, and the costimulatory molecules CD80 and CD86. Cells were able to differentiate into mesenchymal cell types (adipocytes, osteoblasts, chondrocytes, and smooth muscle cells), but also towards endothelium. ${ }^{4}$ Prior to injection, MAPCs were thawed and washed twice and suspended in phosphate buffered saline (PBS) at a concentration of $10 \times 10^{6}$ cells $/ \mathrm{mL}$. MAPCs were administered intravenously 1 hour and 4 days after (sham) UCO. Fetuses received two doses of $10 \times 10^{6}$ cells in $1 \mathrm{~mL}$ PBS.

\section{Immunohistochemistry in the brain}

After 3 months of submersion fixation in $4 \%$ paraformaldehyde, the right cerebral hemisphere was embedded in gelatin and serial coronal sections $(50 \mu \mathrm{m})$ were cut on a Leica VT 1200 S vibrating microtome (Leica Biosystems, Nussloch, Germany). Free floating sections at the level of mid-thalamus and posterior hippocampus were stained with a rabbit anti-ionized calcium binding adaptor molecule 1 (IBA-1) antibody (Wako Pure Chemical Industries, Osaka, Japan) for microglia or a rat anti-myelin basic protein (MBP) antibody (Merck Millipore, Billerica, MA, USA) for myelin sheaths and myelin producing (mature) oligodendrocytes, and cleaved Caspase-3 for apoptosis (Cell Signaling Technology, Boston, USA) as previously described. ${ }^{14,24}$

Briefly, endogenous peroxidase-activity was blocked by incubation with $0.3 \% \mathrm{H}_{2} \mathrm{O}_{2}$ in Tris Buffered Saline (TBS, pH 7.6). Free floating sections were incubated overnight at $4^{\circ} \mathrm{C}$ with the diluted primary antibody (1:1000 anti-IBA-1, 1:2000 MBP, 1:800 Caspase-3) followed by 
incubation with the appropriate secondary antibodies. The immunostaining was enhanced with Vectastain ABC peroxidase Elite kit (PK-6200, Vector Laboratories, Burlingame, CA, USA) followed by a nickel sulfate-diaminobenzidine (IBA-1) or 3,3'-diamniobenzidine staining (MBP, Caspase-3). Sections were mounted on gelatin-coated glass slides, air-dried, dehydrated in ascending ethanol concentrations and coverslipped with PerTex.

\section{Analysis of immunohistochemistry}

Immunohistochemical stainings were analyzed as previously described, for the analysis of IBA-1 and MBP immunoreactivity ${ }^{14}$ and densities of Caspase-3 positive cells. For the analysis of IBA-1 and MBP immunoreactivity (IR), digital images of the subcortical white matter (SCWM) (100x magnification) and hippocampus (20x magnification; only IBA-1) were acquired using an Olympus AX-70 microscope (Olympus, Tokyo, Japan) equipped with a black and white digital camera. In the hippocampus, area fraction of IBA-1 immunoreactivity was assessed in one digital image per section by delineating the hippocampus and determining the areal fraction of IBA-1 immunoreactivity expressed as a percentage of total hippocampal area with a standard threshold using Leica Qwin Pro V 3.5.1 software (Leica, Rijswijk, the Netherlands). In the SCWM, the area fractions of IBA-1 and MBP immunoreactivity were determined in five adjacent 100x digital images obtained in standardized locations within the SCWM of each section. The results of five images per section were averaged to obtain the areal fractions of IBA- 1 and MBP immunoreactivity within the SCWM for each section.

For analysis of cleaved Caspase-3, 5 adjacent digital images (100x magnification) of the SCWM were obtained in standardized locations for each section. Numbers of Caspase-3 positive cells were determined with Leica Qwin Pro V 3.5.1 software, and averaged to obtain cell numbers for Caspase-3 per section.

\section{RNA extraction and quantitative real-time PCR}

For real-time (RT) quantitative polymerase chain reactions (qPCR), total RNA was extracted from spleen using the SV Total RNA isolation system (Z3100; Promega, Madison, WI) according to the manufacturer's recommendations. RT qPCR reactions were performed in duplicate with the SensiMix SYBR No-ROX kit (QT650-02; Bioline Reagents Ltd) in a LightCycler 480 Instrument with ovine specific primers for interleukin IL-10 (5'-CATGGGCC TGACATCAAGGA-3' (sense), 5'-CGGAGGGTCTTCAGCTTCTC-3' (antisense), tumor necrosis factor alpha (TNFa) (5'-GCCGGAATACCTGGACTATGC-3' (sense), 5'-CAGGGCGATGATCCCAAA GTAG-3' (antisense), and interferon gamma (IFNY) (5'- TCAAGCAAGACATGTTCAGAAGTTCT3' (sense), 5'-CCGGAATTTGAATCAGCCTTTTGAA-3' (antisense). RT qPCR results were normalized to the housekeeping gene ovine 40S ribosomal protein S15 (ovRPS15) (5'CGAGATGGTGGGCAGCAT-3' (sense), 5'-GCTTGATTTC CACCTGGTTGA-3' (antisense)). 


\section{MAPC detection}

Detection of MAPC was performed with a PCR for human $\beta-2$ microglobulin. ${ }^{2}$ Genomic DNA was isolated from snap frozen brain, spleen and lung tissues using the Wizard Genomic Purification Kit (Promega, Leiden, The Netherlands) according to the manufacturer's recommendations. PCR reactions of genomic DNA were performed for $\beta-2$ microglobulin followed by a nested PCR for $\beta-2$ microglobulin on the amplification product of the first PCR.

The amplified products were separated on ethidium bromide-stained $2 \%$ agarose gels and captured using the Imagemaster ${ }^{\circledR}$ VDS equipped with a CCD camera (GE Healthcare Life Sciences (Pharmacia Biotech), Uppsala, Sweden). Serial dilutions of MAPCs were used as references.

Primers used for the amplification of $\beta-2$ microglobulin were $5^{\prime}$ - GTGTCTGGGTTTCAT CAATC-3' (sense), 5'- GGCAGGCATACTCATCTTTT-3' (antisense), 5'- TGGGTTTCATCAA TCCGACAT-3' (nested sense) and 5'- CTCATCTTTTTCAGTGGGGGT-3' (nested antisense).

\section{Statistics}

Summary statistics of animal characteristics and all outcome variables are shown as means with 95\% Confidence Interval (CI). Groups' comparisons of all outcome parameters were drawn with analysis of variance (ANOVA) or with random intercept models in case of repeated measurements per animal (e.g. different sections per brain). HI (sham vs. HI) and treatment (saline vs. MAPC) were the fixed effects. For random intercept models, animals constituted additionally the random effect. Variables, whose distributions were positively skewed, were log-transformed previous to statistical testing. To facilitate interpretation, averages on the log scale were back transformed to the original scale (antilog) and were presented as geometric means and corresponding 95\% Cls.

Seizure data, measured over time, showed pronounced right-skewness (caused by the absence of seizures in non-hypoxic conditions) that could not be remedied by log transformation. Hence, for these variables, pair-wise groups' comparisons were performed with nonparametric Mann-Whitney tests, per individual time-point. They are presented as medians and corresponding interquartile range (IQR). A False Discovery Rate (FDR) of $5 \%$ was used for multiple testing correction. Groups' differences with FDR corrected $\mathrm{P}<0.05$ were considered statistically significant. Statistical analysis was performed with IBM SPSS Statistics Version 20.0 (IBM Corp., Armonk, NY, USA).

Statistical analysis of baroreflex data was performed using a Bayesian multilevel model ${ }^{25}$ to estimate the daily effects of global $\mathrm{HI}$ and MAPC treatment under hypoxic and sham conditions on the baroreceptor reflex sensitivity. This model corrects for repeated measurements and takes into account variance between and within subjects. ${ }^{25}$ 
Statistical time series analysis was conducted using the Stan for $\mathrm{R}$ package (version 2.6.0) in $\mathrm{R}$ (version 3.1.1).

\section{RESULTS}

\section{Animal characteristics}

To test the neuroprotective potential of clinical-grade MAPCs, we randomized 32 preterm sheep fetuses in four different experimental groups (Figure 1). After instrumentation and a recovery period of four days, animals were subjected to $25 \mathrm{~min}$ of (sham) umbilical cord occlusion (UCO) to induce global hypoxia-ischemia (HI). MAPCs were administered 1 hour and 4 days after UCO (Figure 1). Fetal body weight and gestational age at did not differ significantly between the four experimental groups (Table 1). In line with previous reports ${ }^{2,14,15}$, the fetal response to hypoxia-ischemia was comparable in all animals exposed to UCO (Figure 2), indicating that the injury group (HI-SAL) and intervention group (HI-MAPC) were exposed to a similar degree of global hypoxia-ischemia.

Table 1. Animal characteristics.

\begin{tabular}{lllll}
\hline & sham-SAL & sham-MAPC & HI-SAL & HI-MAPC \\
\hline GA at UCO $(\mathrm{d})$ & 105.2 & 106.0 & 105.5 & 105.2 \\
& $(105.0 ; 105.5)$ & $(105.3 ; 106.7)$ & $(105.1 ; 106.0)$ & $(104.9 ; 105.8)$ \\
BW $(\mathrm{g})$ & 1690 & 1532 & 1542 & 1559 \\
& $(1516 ; 1865)$ & $(1245 ; 1820)$ & $(1362 ; 1721)$ & $(1340 ; 1779)$ \\
Brain weight $(\mathrm{g} / \mathrm{kg} \mathrm{BW})$ & 17.58 & 17.39 & 15.97 & 16.08 \\
& $(16.04 ; 19.12)$ & $(14.72 ; 20.05)$ & $(14.43 ; 17.51)^{*}$ & $(14.37 ; 17.80)$ \\
\hline
\end{tabular}

Fetuses were subjected to umbilical cord occlusion (UCO) at a comparable gestational age (GA). Fetal body weight (BW) did not differ between experimental groups. Brain weight corrected for bodyweight was significantly reduced following global HI. MAPC treatment did not prevent loss of cerebral weight. Means \pm 95\% $\mathrm{Cl}$ are shown. Groups' comparisons were drawn with ANOVA (sham-SAL $n=8$, sham-MAPC $n=8, \mathrm{HI}-\mathrm{SAL}$ $\mathrm{n}=8, \mathrm{HI}-\mathrm{MAPC} \mathrm{n}=8) * \mathrm{P} \leq 0.05$. $\mathrm{HI}=$ hypoxia-ischemia, $\mathrm{SAL}=$ saline, $\mathrm{MAPC}=$ multipotent adult progenitor cells.

\section{MAPCs reduced the cerebral inflammatory response after global $\mathrm{HI}$}

The cerebral inflammatory response was studied by assessing microglial proliferation in the subcortical white matter and hippocampus using ionized calcium binding adaptor molecule 1 (IBA-1), which is a highly specific marker for resting and activated microglia in sheep. ${ }^{14}$ Global HI resulted in a significant (sham-SAL vs. HI-SAL; P<0.001) increase of IBA-1 immunoreactivity in the SCWM and hippocampus, indicating profound microglial proliferation in these regions (Figure 3A-F). MAPCs significantly (HI-SAL vs. HI-MAPC; $\mathrm{P}=0.043$ ) reduced IBA-1 immunoreactivity in the SCWM (Figure 3A-C). MAPCs did not 
reduce IBA-1 immunoreactivity in the hippocampus (HI-SAL vs. HI-MAPC; P=0.881) (Figure 3D-F). No differences in IBA-1 immunoreactivity were observed between saline or MAPC treated sham operated animals in the SCWM and hippocampus.
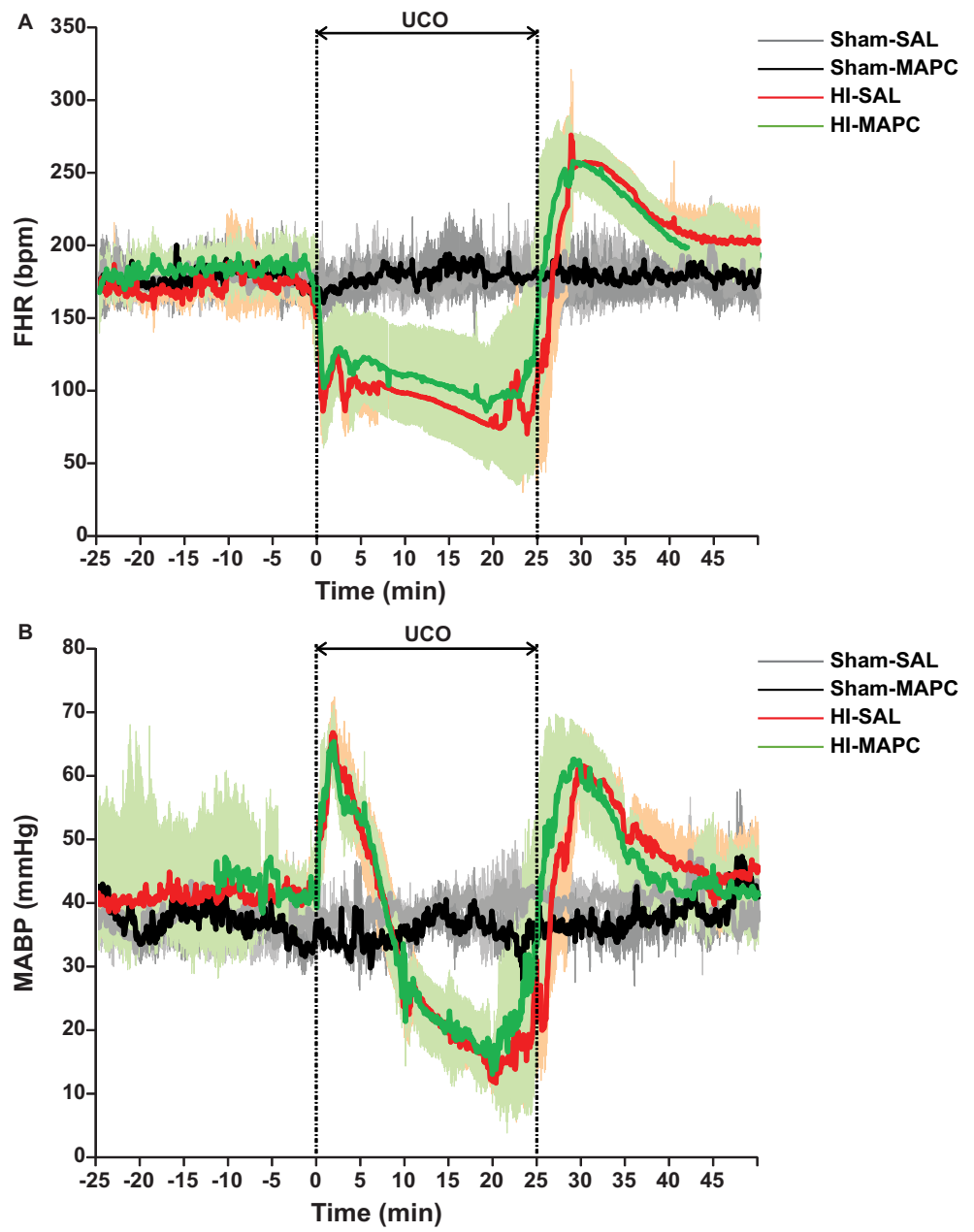

Figure 2. Reproducibility of 25 min umbilical cord occlusion (UCO) as evidenced by comparable vital parameters and blood gases in animals exposed to global HI. (A)

Fetal heart rate (FHR) and (B) fetal mean arterial blood pressure (MABP) measurements indicated that all animals exposed to global $\mathrm{HI}$ experienced the same degree of bradycardia and hypotension, respectively; means (thick line) \pm SD (shaded areas) of $n=8$ animals per experimental group are shown. $\mathrm{HI}=$ hypoxiaischemia, $\mathrm{SAL}=$ saline, $\mathrm{MAPC}=$ multipotent adult progenitor cells, $\mathrm{min}=$ minutes 

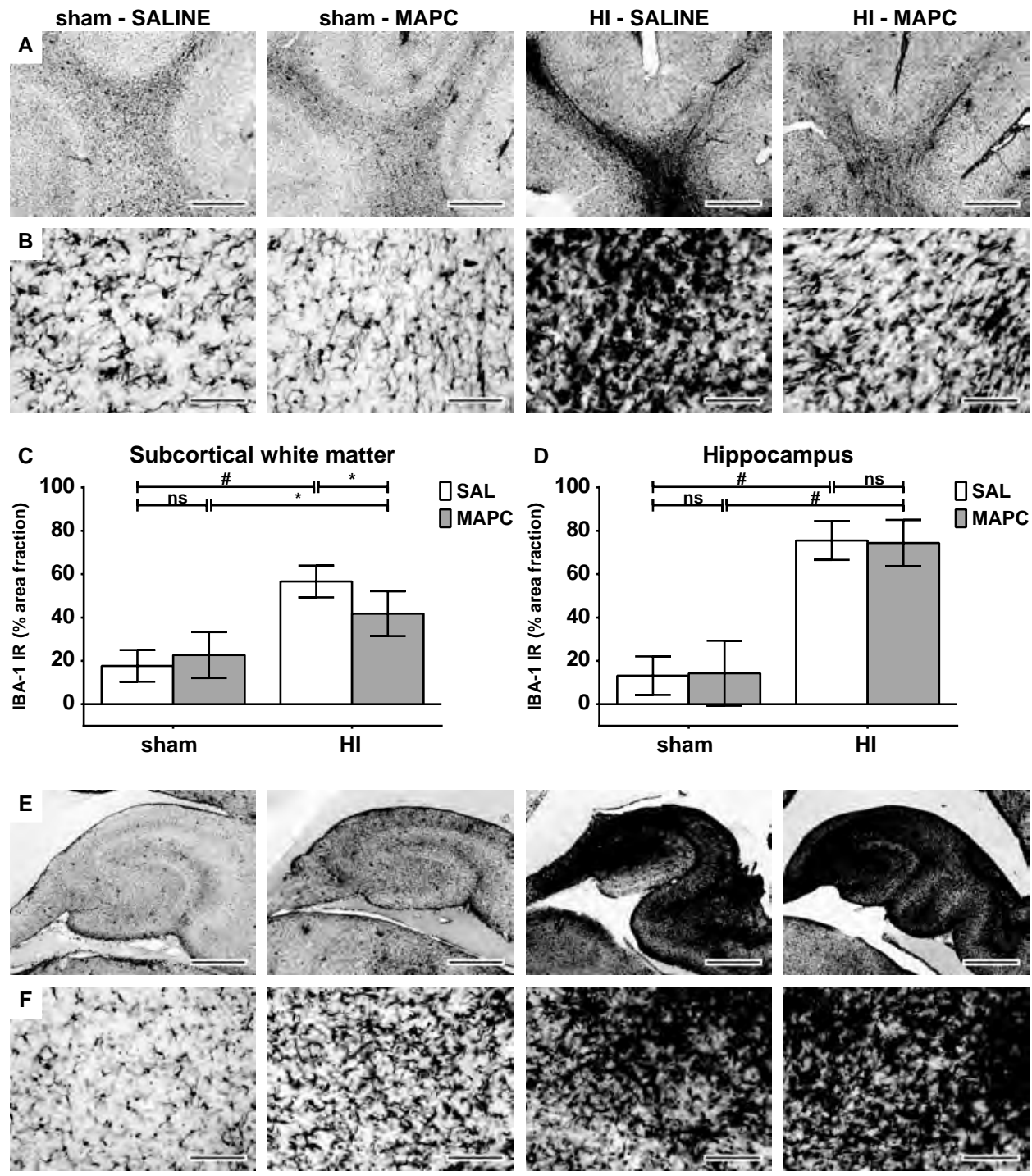

Figure 3. MAPCs reduced cerebral inflammation in the subcortical white matter (SCWM), but not in the hippocampus. (A-B)

Immunohistochemical IBA-1 staining in the SCWM at 20x (A) and 200x (B) magnification of the four experimental groups (sham-SAL $n=8$, sham-MAPC $n=5, H I-S A L n=8, H I-M A P C n=5$ ). Global HI induced a profound increase of IBA1 immunoreactivity and amoeboid morphology, which was significantly reduced by MAPCS in the SCWM. (C-D) Graphical presentation of area fraction of IBA-1 immunoreactivity in SCWM and hippocampus. Geometric means \pm $95 \% \mathrm{Cl}$ and levels of significance are depicted, which were calculated by the random intercept model with all repeated measures (i.e. brain sections) per animal. (E-F) Immunohistochemical IBA-1 staining in the hippocampus at 20x (E) and 200x (F) magnification of the four experimental groups. IBA-1 IR was greatly increased and accompanied with amoeboid morphology following global $\mathrm{HI}$, which remained unaffected after MAPC treatment. * $\mathrm{P} \leq 0.05, \S \mathrm{P} \leq 0.01, \# \mathrm{P} \leq 0.001$. IBA-1 = ionized calcium binding adaptor molecule $1, \mathrm{HI}=$ hypoxia-ischemia, $\mathrm{SAL}=$ saline, $\mathrm{MAPC}=$ multipotent adult progenitor cells, $\mathrm{IR}=$ immunoreactivity. Scale bars: (A-E) $1 \mathrm{~mm}$, (B-F) $100 \mu \mathrm{m}$. 
MAPCs prevented HI-induced white matter injury

White matter injury was studied by assessing myelin basic protein (MBP) immunoreactivity in the SCWM. Global HI significantly (sham-SAL vs. HI-SAL; P<0.001) reduced $\mathrm{MBP}$ immunoreactivity in the SCWM, indicating $\mathrm{HI}$-induced hypomyelination in this region (Figure $4 \mathrm{~A}-\mathrm{C}$ ). MAPCs significantly increased MBP immunoreactivity (HI-SAL vs. HI-MAPC; $P=0.016)$ No differences in MBP immunoreactivity in the SCWM were observed between sham animals treated with saline or MAPCs (Figure 4A-C). We determined brain weight as a surrogate for neuronal injury (table 1). Brain weight was significantly reduced after global HI (sham-SAL vs. HI-SAL; P=0.046). MAPCs did not prevent loss of brain weight (HI-SAL vs. HI-MAPC; P=0.429).

Apoptotic cell death was evaluated in the SCWM by assessment of the number of cleaved Caspase-3 positive cells (Figure 4D). No differences in numbers Caspase-3 positive cells were found 7 days after global HI (sham-SAL vs. HI-SAL; P=0.135). The density of apoptotic cells remained unaffected by systemic administration of MAPCs (HI-SAL vs. HI-MAPC; P=0.881).

We did not detect MAPCs in brain, spleen or lung tissue 7 days after global HI (i.e. 3 days after the second dose of MAPCs) using PCR for human-specific $\beta-2$ microglobulin (data not shown).

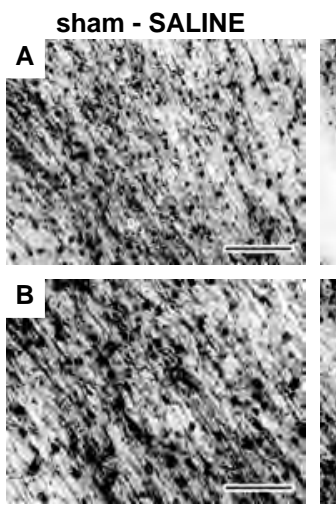

sham - MAPC
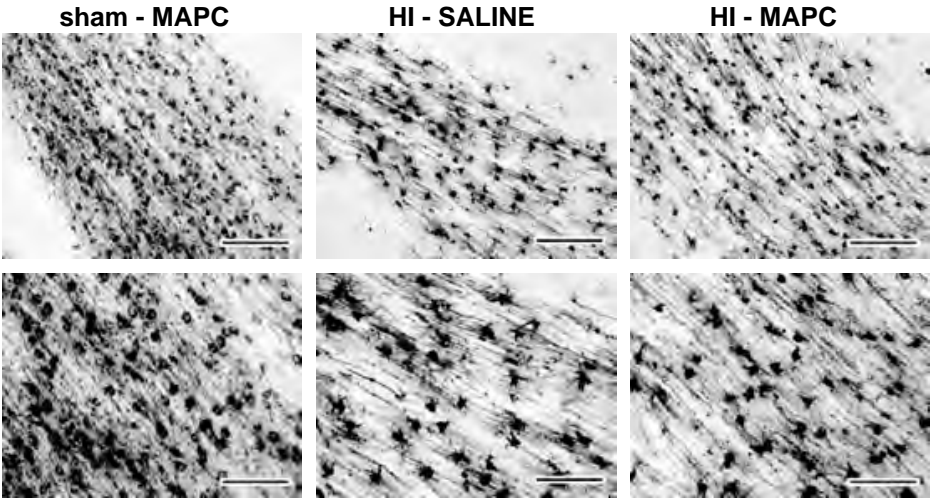

C

Subcortical white matter

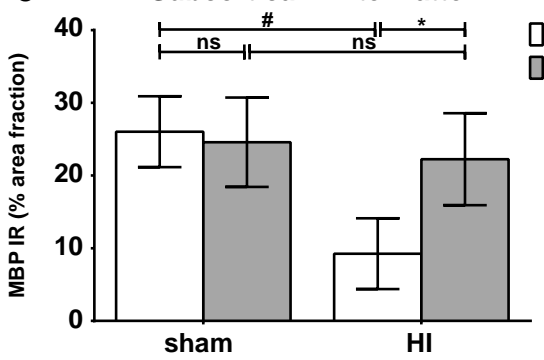

D

TSAL TMAPC

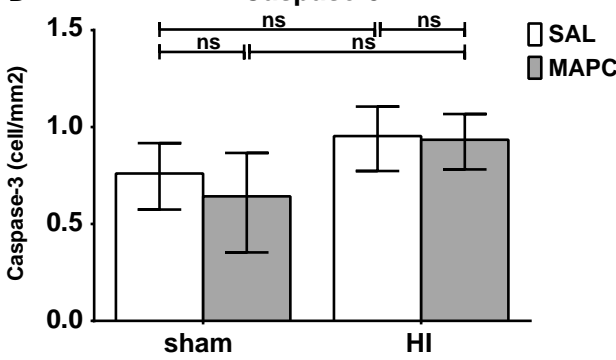


Figure 4. MAPCs reduced white matter injury after global HI.

(A-B) Immunohistochemical MBP staining in the SCWM of the four experimental groups at (A) $100 \mathrm{x}$ and (B) 200x magnification. (C) MBP immunoreactivity in SCWM. Global HI induced marked hypomyelination in the SCWM. MAPCs significantly prevented the decrease in MBP reactivity after global HI. The area fraction of MBP was similar in sham conditions. Geometric means $\pm 95 \% \mathrm{Cl}$ and levels of significance are depicted, which were calculated by the random intercept model with all repeated measures (i.e. brain sections) per animal (shamSAL $n=8$, sham-MAPC $n=5, H I-S A L n=8, H I-M A P C n=5$ ). (D) Numbers of Caspase-3 positive cells in the four experimental groups; geometric means $\pm 95 \% \mathrm{Cl}$ and levels of significance are depicted, which were calculated with ANOVA (sham-SAL $n=8$, sham-MAPC $n=8$, HI-SAL $n=8$, HI-MAPC $n=8$ ). * P $\leq 0.05, \S P \leq 0.01$, \# $\mathrm{P} \leq 0.001$. $\mathrm{MBP}=$ myelin basic protein $1, \mathrm{HI}=$ hypoxia-ischemia, $\mathrm{SAL}=$ saline, $\mathrm{MAPC}=$ multipotent adult progenitor cells, IR = immunoreactivity. Scale bars: (A) $200 \mu \mathrm{m}$, (B) $100 \mu \mathrm{m}$.

\section{MAPCs prevented splenic involution and modulated the peripheral inflammatory response}

Splenic weight was assessed, since activation of the splenic inflammatory response was previously shown to be characterized by mobilization of effector immune cells affecting volume and weight of the spleen. ${ }^{14} \mathrm{Global} \mathrm{HI}$ induced splenic involution in saline treated animals (sham-SAL vs. HI-SAL, P=0.022) (Figure 5A). MAPCs significantly prevented splenic involution after global $\mathrm{HI}$ (HI-SAL vs. HI-MAPC, P=0.032). The splenic cytokine profile was studied since previous findings indicated that MAPCs established neuroprotection by modulating the splenic inflammatory response (Figure 5B-D). Splenic IL-10 mRNA levels were significantly increased after global HI (sham-SAL vs. HI$\mathrm{SAL}, \mathrm{P}=0.031$ ). MAPCs significantly reduced IL-10 mRNA levels in the spleen after global $\mathrm{HI}$ (HI-SAL vs. HI-MAPC, P=0.042) (Figure 5B). IFNy mRNA levels were significantly increased following global $\mathrm{HI}$ (sham-SAL vs. HI-SAL; $\mathrm{P}=0.022$ ). Systemic administration of MAPCs tended to reduce IFNy mRNA levels, but statistical significance was not reached (HI-SAL vs. HI-MAPC; $\mathrm{P}=0.078$ ) (Figure $5 \mathrm{C}$ ). Global $\mathrm{HI}$ tended to increase splenic TNF $\alpha$ mRNA levels (sham-SAL vs. HI-SAL; P=0.078). However, MAPC treatment significantly reduced TNF $\alpha$ mRNA levels (HI-SAL vs. HI-MAPC; P<0.001) (Figure 5D). 
A

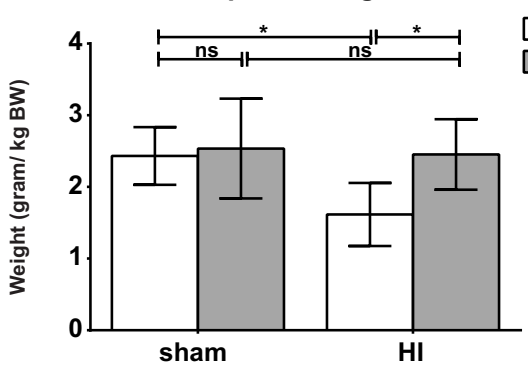

C

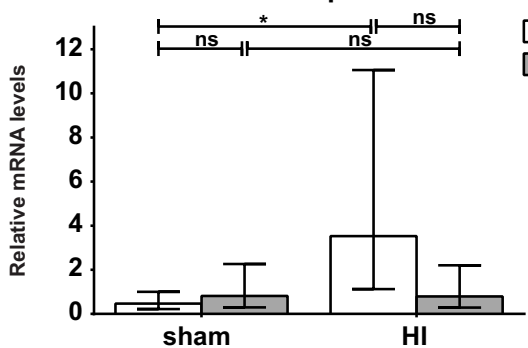

B

TSAL

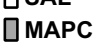

TSAL

DMAPC
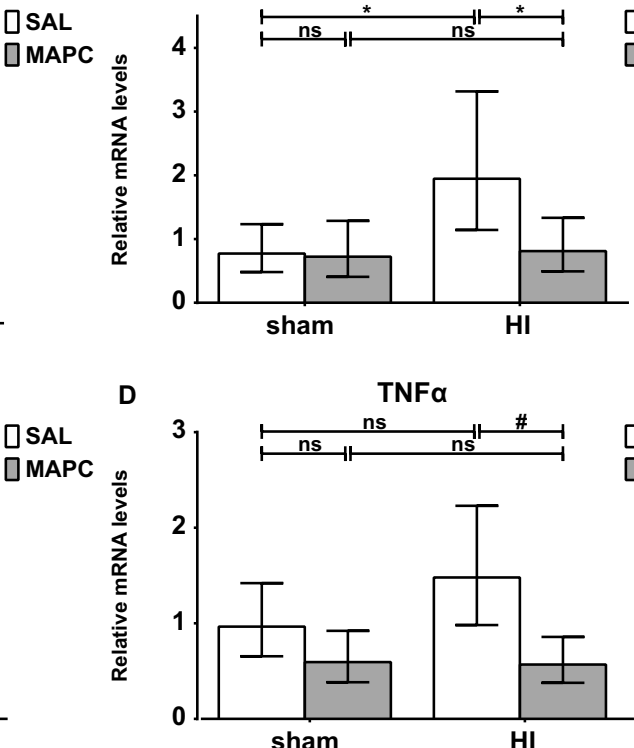

D

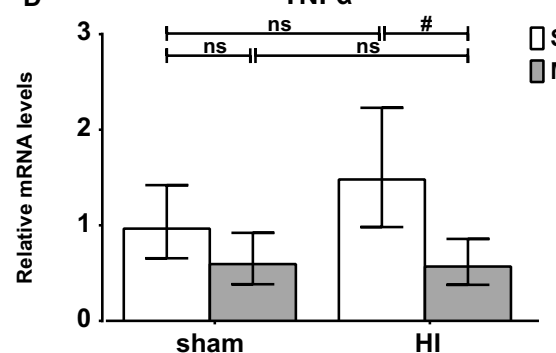

Figure 5. MAPCs prevented splenic involution and modulated the peripheral inflammatory response. (A) Splenic weight corrected for body weight (BW).

Global $\mathrm{HI}$ induced significant splenic involution, which was significantly prevented by MAPCs; means $\pm 95 \% \mathrm{Cl}$ and levels of significance are depicted, which were calculated with ANOVA (sham-SAL $n=8$, sham-MAPC $n=8$, HI-SAL n=8, HI-MAPC n=8). (B) IL-10 mRNA levels were significantly increased in the spleen 7 days after global HI. MAPCs significantly attenuated the HI-induced IL-10 response in the spleen. (C) Splenic IFNy mRNA levels were significantly increased 7 days following global HI. MAPC treatment tended to reduce IFNץ mRNA levels. However, statistical significance was not reached. (D) TNF $\alpha$ mRNA levels showed a trend to increase following global $\mathrm{HI}$, which was significantly prevented by MAPCs; geometric means $\pm 95 \% \mathrm{Cl}$ and levels of significance are depicted, which were calculated with ANOVA (sham-SAL $n=8$, sham-MAPC n=6, HI-SAL n=8, HI-MAPC $n=8$ ). * $P \leq 0.05, \S P \leq 0.01, \# P \leq 0.001$. $H I=$ hypoxia-ischemia, $S A L=$ saline, $M A P C=$ multipotent adult progenitor cells, IFN $\gamma=$ interferon gamma, TNF $\alpha=$ tumor necrosis factor alpha.

\section{MAPCs reduced seizure burden after global HI}

Electrographic seizure activity was assessed to determine the effect of MAPCs on cortical function after global $\mathrm{HI}$ (Figure 6A-B). Global HI induced a significant increase in total number (Figure 6A) and duration (Figure 6B) of seizures after global HI (sham-SAL vs. HI-SAL, significant in all time points). Seizure burden peaked within the first 48 hours following global $\mathrm{HI}$ with a second small rise in activity around experimental day 4 and 5 (Figure 6A-B). MAPCs reduced seizure burden throughout the complete reperfusion period (HI-SAL vs. HI-MAPCS, significant from experimental day 2 onwards). Cumulative number of seizures in the complete reperfusion study was significantly increased after global HI (sham-SAL vs. HI-SAL, $\mathrm{P}=0.016$ ) and significantly reduced by MAPCS (HI-SAL vs. HI-MAPC, $P=0.022$ ) (Figure 6A). Similarly, cumulative duration (sec) of seizures was 
significantly increased after global HI (sham-SAL v. HI-SAL, $\mathrm{P}=0.015$ ) and significantly reduced by MAPCS (HI-SAL vs. HI-MAPC, P=0.043) (Figure 6B). No electrographic seizure activity was detected under sham conditions.
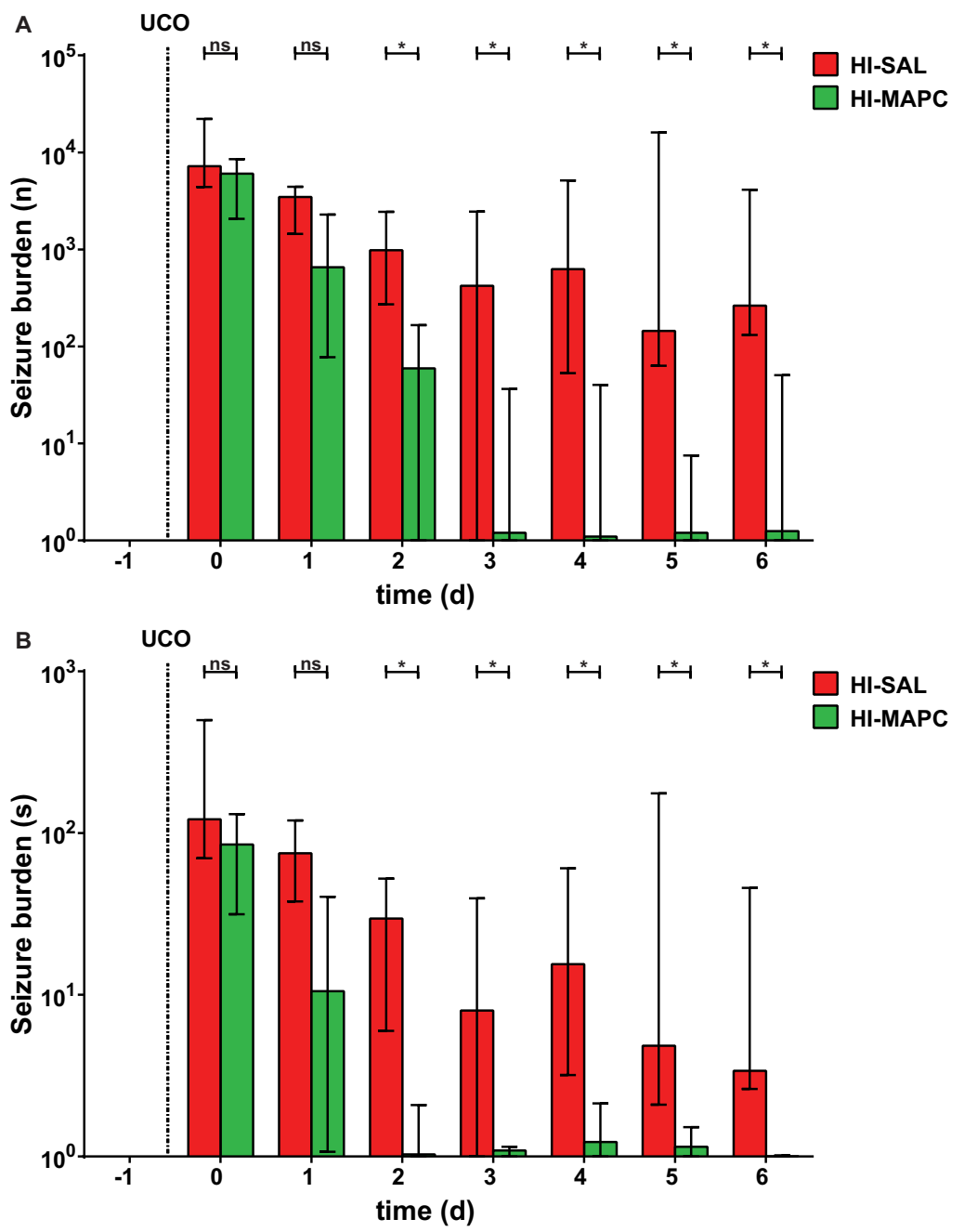

Figure 6. MAPCs induced functional neuroprotection after global $\mathrm{HI}$.

Global HI caused a significant seizure burden indicated by an increased (A) total number and (B) duration of seizures compared to controls. Administration of MAPCs significantly reduced electrographic seizure number and duration. Medians \pm interquartile ranges (IQR) and levels of significance of the treatment effect (HI-SAL vs. HI-MAPC) are depicted, which were calculated by Mann-Whitney test (HI-SAL $n=8$, HI-MAPC $n=8$ ). No electrographic seizure activity was detected under sham conditions. For readability purposes these sham groups (sham-SAL $n=8$ and sham-MAPC $n=6$ ) are not shown. ${ }^{*} P \leq 0.05, \S P \leq 0.01, \# P \leq 0.001$. HI = hypoxiaischemia, $\mathrm{SAL}=$ saline, $\mathrm{MAPC}=$ multipotent adult progenitor cells 


\section{MAPCs prevented loss of baroreceptor reflex sensitivity after global HI}

Baroreceptor reflex sensitivity was analyzed to study the effect of MAPC treatment on deeper brain functions situated in the brain stem (Figure 7). Global HI increasingly compromised baroreceptor reflex sensitivity over time (sham-SAL vs. HI-SAL, significant for all time points with the exception of experimental day 1). MAPCs significantly prevented the loss of baroreceptor reflex sensitivity (HI-SAL vs. HI-MAPC, significant on all time points).

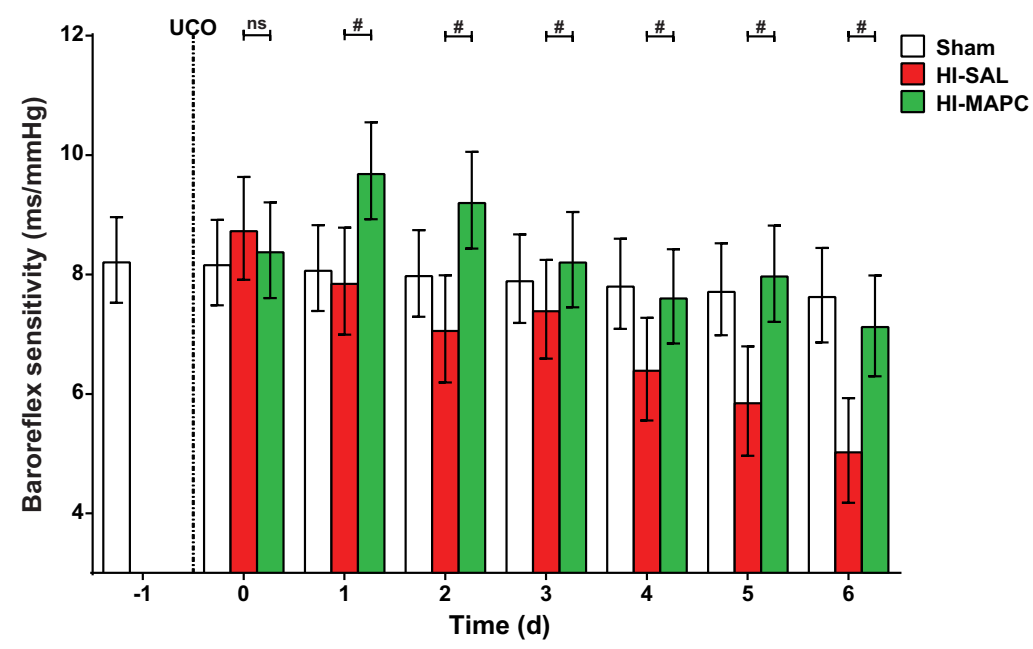

Figure 7. MAPCs prevented loss of baroreceptor reflex sensitivity.

Global HI caused a significant gradual decline of baroreceptor reflex sensitivity over time, which was prevented by MAPC treatment; means $\pm 95 \% \mathrm{Cl}$ and levels of significance of the treatment effect (HI-SAL vs. HI-MAPC) are depicted, which were calculated by the Bayesian multi-level model. There were no differences in baroreceptor reflex sensitivity between the sham-SAL and sham-MAPC groups. For readability purposes all sham treated animals (sham-SAL $n=8$ and sham-MAPC $n=6$ ) were grouped and depicted as one sham group. HI-SAL $n=8, H I-M A P C n=8 . * P \leq 0.05, \S P \leq 0.01, \# P \leq 0.001 . H I=$ hypoxia-ischemia, $S A L=$ saline, $M A P C=$ multipotent adult progenitor cells.

\section{DISCUSSION}

Hypoxic-ischemic brain injury is common in preterm infants. To date no treatment options are available to reduce mortality and morbidity associated with brain injury in preterm infants. Our group has previously shown in a well-established pre-clinical model of global hypoxic-ischemia (HI) that mesenchymal stromal cells are a promising candidate for the treatment of hypoxic-ischemic brain injury. ${ }^{2}$ In the current study we investigated the neuroprotective capacity of multipotent adult progenitor cells (MAPCs), which are a subset of bone marrow-derived MSCs with more potent anti- 
inflammatory and regenerative properties. 3, 4, 6-10 We showed that intravenous administration of MAPCs prevented functional and structural injury in the preterm brain and modulated the cerebral and peripheral inflammatory response after global $\mathrm{HI}$.

We administered MAPCs 1 hour and 4 days after global HI. With the first dose we aimed to dampen the acute cerebral and peripheral inflammatory response, which has been shown to evolve early after global hypoxia-ischemia. ${ }^{2}$ A pilot study in this model showed a secondary peak in seizure burden 4-5 days after global $\mathrm{HI}$ (unpublished data). Therefore, the second dose of MAPCs was administered 4 days after global $\mathrm{HI}$ aimed at preventing secondary functional injury.

Our findings showed that MAPCs 1 hour and 4 days after global HI reduced seizure burden after global $\mathrm{HI}$ throughout the study period of 7 days. This finding is clinically highly relevant, since several studies have shown that seizures in term and preterm neonatal HIE are associated with adverse neurodevelopmental outcome. ${ }^{26-28}$ In our model we detected seizure activity with amplitude-integrated EEG (aEEG), which is clinically used to monitor neonatal brain function and has a high sensitivity and specificity in predicting neurodevelopmental outcomes in neonates with $\mathrm{HI}$ brain injury. ${ }^{29}$ Recorded seizure activity in our study was most likely located in the cortical layers. However, since the electrodes were placed directly on the dura, we may have recorded seizures or electrophysiological responses originating from deeper regions underlying the cortex (i.e. thalamus and hippocampus). In addition to cortical grey matter protection, the reduced seizure activity may therefore also represent protection of deeper brain structures. We therefore assessed the baroreceptor reflex as a function of the brain stem. This is clinically of importance since the baroreflex is a vital part of the cardiovascular auto regulatory system in preterm infants, which secures adequate perfusion of the preterm brain. ${ }^{22}$ The cerebrovascular system is still immature in prematurity making the preterm brain extremely vulnerable to fluctuations in blood pressure caused by $\mathrm{HI}$ (or other adverse events such as infection). ${ }^{30}$ Previously, we demonstrated that global HI disturbed normal development of the baroreflex, which resulted in impairment of heart rate mediated blood pressure control. ${ }^{22}$ The fact that MAPC treatment at $1 \mathrm{~h}$ and 4 days improved baroreceptor reflex sensitivity indicated that the therapeutic effects reach as far as the highly conserved central brain functions in the brainstem and supports the concept of MAPC-induced protection against grey matter injury in the hypoxic-ischemic preterm brain. The protection of grey matter by MAPCs after global HI may be explained by findings of previous reports demonstrating that MAPCs improved neurogenesis and stimulated neurite outgrowth and synaptogenesis. ${ }^{7,31,32}$

Density of apoptotic cells in the subcortical white matter was neither affected by global $\mathrm{HI}$ nor by MAPC treatment in this study. The fact that we did not detect significant changes in cell death is most likely explained by timing of assessment at 7 days after global HI, while apoptotic cell death peaks earlier after cerebral hypoxia-ischemia. 
Further studies on the dynamics of cell death in the preterm brain after global $\mathrm{HI}$ are needed to determine the therapeutic effect of MAPCs on neuronal loss.

MAPCs have been previously shown to have potent anti-inflammatory properties in preclinical studies of traumatic and ischemic injury of the central nervous system. ${ }^{6-11}$ Our study confirmed and extended these findings in a translational model of hypoxicischemic injury in the preterm brain by showing that MAPCs reduced the neuroinflammatory response in the subcortical white matter (SCWM), as evidenced by reduced microglial proliferation. We and others have shown that the microglial response is a reliable marker for the cerebral inflammatory response that plays a pivotal role in the etiology of hypoxic ischemic encephalopathy in the preterm brain. ${ }^{14,33}$ Although the size of the treatment effect was modest, reduction of the microglial response was associated with functional and structural improvement, indicating the biological relevance of modulating the cerebral inflammatory response mediated by microglia. We postulate that the observed MAPC-induced reduction of cerebral inflammation after global HI may be explained by two mechanisms; firstly, MAPCs have been shown to induce a microglial type switch from a pro-inflammatory M1 phenotype to an anti-inflammatory M2 phenotype, which is associated with reduced inflammation in the CNS. ${ }^{8}$ Secondly, MAPCS may have reduced glutamate excitotoxicity in a similar fashion as MSCs, which were shown previously to inhibit glutamate receptor expression and function, thereby inhibiting microglial activation ${ }^{34}$. However, MAPCs did not attenuate the inflammatory response in the hippocampus. These spatial differences in anti-inflammatory effects are in line with previous data showing that the hippocampus is more severely affected by hypoxia-ischemia and therefore injury may be irreversible. ${ }^{2,14,15}$

White matter injury is the clinical hallmark of $\mathrm{HI}$ brain injury in the preterm brain. ${ }^{35}$ Consistent with earlier studies ${ }^{2}$ 14, 15, we demonstrated that global hypoxia-ischemia induced marked hypomyelination of the preterm brain, which was prevented by intravenous administration of MAPCs. Microglial type switch and subsequent reduction of cerebral inflammation has been previously associated with remyelination and may therefore explain the observed reduction of white matter injury. ${ }^{36,37}$

Besides attenuation of the cerebral inflammatory response, our findings indicated that MAPCs modulated the peripheral inflammatory response, which has been implicated in the etiology of hypoxic-ischemic injury in the preterm brain. ${ }^{14,38-40}$ Our results showed that MAPCs prevented splenic involution. We have previously shown in the current model that splenic involution after global HI was caused by efflux of immune effector cells. ${ }^{14}$ Furthermore, our findings demonstrated that intravenous administration of MSCs induced splenic T-cell tolerance. ${ }^{2}$ The observed unresponsiveness of splenic Tcells was associated with reduced systemic mobilization of immune effector cells and concomitant reduction of brain infiltrating immune cells resulting in less severe inflammation in the preterm brain after global $\mathrm{HI}^{2}$ Based on these findings, we 
postulate that MAPCs reduced the mobilization of immune effector cells from the spleen resulting in less extensive invasion of these injurious cells into the brain. This concept is supported by previous findings showing a spleen-mediated neuroprotective effect of MAPCs in traumatic CNS injury. ${ }^{9,11}$ Recently, it was demonstrated that splenic IFNy plays a key role in injurious signaling to the ischemic brain. ${ }^{41}$ Functional elimination by splenectomy or administration of IFNY neutralizing antibodies reduced brain injury after stroke. ${ }^{42}$ In line with these findings, reduction of cerebral inflammation (i.e. microglial proliferation) by MAPCs was associated with decreased splenic IFNy levels. To determine whether IFNY from splenic cells is involved in preterm brain injury after global $\mathrm{HI}$, temporal dynamics of splenic IFNy release needs to be elucidated and its functional role to be determined.

We cannot confirm the IL-10 mediated therapeutic effect of MAPCs inducing increased splenic and systemic numbers of protective regulatory T-cells (Tregs) in adult animals. ${ }^{9}$ In our study splenic IL-10 mRNA levels were not altered by MAPCs in sham conditions. Instead, we found a marked increase of IL-10 mRNA under hypoxic-ischemic conditions, which was attenuated by administration of MAPCs. We postulate that the observed increase of splenic IL-10 mRNA after global $\mathrm{HI}$ is part of an endogenous regenerative response after injury. MAPCs may have reduced the injurious load eliciting such a response resulting in reduced IL-10 levels at the time of assessment ( 7 days after global $\mathrm{HI}$ ). This hypothesis is supported by previous reports showing activation of endogenous repair processes involving IL-10 mediated Treg mobilization after cerebral ischemia. ${ }^{39,40,}$ ${ }^{43}$ Furthermore, early administration of MSCs was shown to prevent progression of hypoxic-ischemic injury. ${ }^{2}$ These findings suggest that early administration of MAPCs prevents injury before endogenous repair mechanisms, such as IL-10 mediated Treg mobilization, are activated.

In line with previous studies reporting the clearance of MAPCs within 48-72 hours $^{\text {10, 31, }}$ we did not detect MAPCs in brain, spleen or lung tissue 7 days after global HI (i.e. 3 days after the second dose of MAPCs). The fact that we found MAPC-mediated therapeutic effects without detectable presence of MAPCs in the brain after 7 days of onset of hypoxia-ischemia, suggests that the neuroprotective effects of MAPCs last longer than their presence. We postulate that this phenomenon can be explained by MAPCs dampening the detrimental peripheral and cerebral inflammatory responses at an early stage, before these responses can aggravate hypoxic-ischemic injury in the preterm brain.

Our study has several limitations. We did not perform time series of histologic analysis to assess temporal dynamics of global HI-induced injury, the cerebral and peripheral inflammatory response and therapeutic effects of intravenous MAPC administration. Moreover we did not discriminate between the therapeutic effect between the first and second dose of MAPCs. These limitations are inherent to limited experimental groups to 
make large animal experiments feasible. Unfortunately, no ovine-specific antibodies are currently available to detect M1 and M2 microglial phenotypes in our studies.

Our findings are clinically highly relevant for several reasons. First, we tested our hypothesis in a well-established pre-clinical animal in which global $\mathrm{HI}$ was induced by transient umbilical cord occlusion accurately mimicking the common etiology of hypoxic-ischemic brain injury in preterm infants. ${ }^{44,45}$ Second, this pre-clinical animal model allows for continuous in utero registration of relevant parameters (i.e. vital parameters and $\mathrm{aEEG}$ ) with strong clinical relevance and predictive value, which gives more insight in $\mathrm{HI}$-induced pathophysiology and efficacy of therapeutic options in a preclinical setting. Third, preclinical studies have confirmed safety and genomic stability of clinical grade MAPCs ${ }^{5,23}$ and clinical-grade MAPC therapy is currently being tested in phase I/II clinical trials for the treatment of ischemic stroke, graft-versus-host disease (GVHD), acute myocardial infarction and inflammatory bowel disease ${ }^{4}$, emphasizing clinical applicability of these cells.

In conclusion, we have shown in a pre-clinical animal model that MAPCs improve the functional and structural outcome of the preterm brain after global HI. Future studies should further establish the mechanism and long-term therapeutic effects of MAPCinduced neuroprotection in the developing preterm brain exposed to hypoxia-ischemia. Our study may form the basis for future clinical trials, which will evaluate whether MAPC therapy is capable of reducing neurological sequelae in preterm infants with hypoxic-ischemic brain injury.

\section{Conclusions}

In conclusion, we have shown in a pre-clinical animal model that MAPCs improve the functional and structural outcome of the preterm brain after global HI. Future studies should further establish the mechanism and long-term therapeutic effects of MAPCinduced neuroprotection in the developing preterm brain exposed to hypoxia-ischemia. Our study may form the basis for future clinical trials, which will evaluate whether MAPC therapy is capable of reducing neurological sequelae in preterm infants with hypoxic-ischemic brain injury. 


\section{REFERENCES}

1. Institute of Medicine (ed). Preterm birth: Causes, Consequences and Prevention. The National Academies Press: Washington, 2007.

2. Jellema, R.K. et al. Mesenchymal stem cells induce T-cell tolerance and protect the preterm brain after global hypoxia-ischemia. PLoS One 8, e73031 (2013).

3. Jacobs, S.A. et al. Human multipotent adult progenitor cells are nonimmunogenic and exert potent immunomodulatory effects on alloreactive T-cell responses. Cell Transplant 22, 1915-1928 (2013).

4. Jacobs, S.A., Roobrouck, V.D., Verfaillie, C.M. \& Van Gool, S.W. Immunological characteristics of human mesenchymal stem cells and multipotent adult progenitor cells. Immunol Cell Biol 91, 32-39 (2013).

5. Kovacsovics-Bankowski, M. et al. Pre-clinical safety testing supporting clinical use of allogeneic multipotent adult progenitor cells. Cytotherapy 10, 730-742 (2008).

6. Bedi, S.S. et al. Intravenous multipotent adult progenitor cell therapy attenuates activated microglial/macrophage response and improves spatial learning after traumatic brain injury. Stem Cells Transl Med 2, 953-960 (2013).

7. Busch, S.A. et al. Multipotent adult progenitor cells prevent macrophage-mediated axonal dieback and promote regrowth after spinal cord injury. J Neurosci 31, 944-953 (2011).

8. Walker, P.A. et al. Intravenous multipotent adult progenitor cell therapy after traumatic brain injury: modulation of the resident microglia population. J Neuroinflammation 9, 228 (2012).

9. Walker, P.A. et al. Intravenous multipotent adult progenitor cell therapy for traumatic brain injury: preserving the blood brain barrier via an interaction with splenocytes. Exp Neurol 225, 341-352 (2010).

10. Yasuhara, T. et al. Intravenous grafts recapitulate the neurorestoration afforded by intracerebrally delivered multipotent adult progenitor cells in neonatal hypoxic-ischemic rats. J Cereb Blood Flow Metab 28, 1804-1810 (2008).

11. DePaul, M.A. et al. Intravenous multipotent adult progenitor cell treatment decreases inflammation leading to functional recovery following spinal cord injury. Scientific reports 5 (2015).

12. Fischer, U.M. et al. Pulmonary passage is a major obstacle for intravenous stem cell delivery: the pulmonary first-pass effect. Stem Cells Dev 18, 683-692 (2009).

13. Back, S.A., Riddle, A. \& Hohimer, A.R. Role of instrumented fetal sheep preparations in defining the pathogenesis of human periventricular white-matter injury. J Child Neurol 21, 582-589 (2006).

14. Jellema, R.K. et al. Cerebral inflammation and mobilization of the peripheral immune system following global hypoxia-ischemia in preterm sheep. J Neuroinflammation 10, 13 (2013).

15. Jellema, R.K. et al. Systemic G-CSF attenuates cerebral inflammation and hypomyelination but does not reduce seizure burden in preterm sheep exposed to global hypoxia-ischemia. Exp Neurol 250C, 293-303 (2013).

16. Niemarkt, H.J. et al. Quantitative analysis of maturational changes in EEG background activity in very preterm infants with a normal neurodevelopment at 1 year of age. Early Hum Dev 86, 219-224 (2010).

17. Niemarkt, H.J. et al. Multi-channel amplitude-integrated EEG characteristics in preterm infants with a normal neurodevelopment at two years of corrected age. Early Human Development 88, 209-216 (2012).

18. Jennekens, W. et al. Automatic burst detection for the EEG of the preterm infant. Physiol Meas 32, 16231637 (2011).

19. Andriessen, P. et al. Cardiovascular fluctuations and transfer function analysis in stable preterm infants. Pediatr Res 53, 89-97 (2003).

20. Andriessen, P. et al. Baroreceptor reflex sensitivity in human neonates: the effect of postmenstrual age. J Physiol 568, 333-341 (2005).

21. Bernardi, L. et al. New method to measure and improve consistency of baroreflex sensitivity values. Clin Auton Res 20, 353-361 (2010).

22. Zwanenburg, A. et al. Heart rate-mediated blood pressure control in preterm fetal sheep under normal and hypoxic-ischemic conditions. Pediatr Res 73, 420-426 (2013). 


\section{Chapter 2}

23. Boozer, S. et al. Global Characterization and Genomic Stability of Human MultiStem, A Multipotent Adult Progenitor Cell. J Stem Cells 4, 17-28 (2009).

24. Kuypers, E. et al. Effects of intra-amniotic lipopolysaccharide and maternal betamethasone on brain inflammation in fetal sheep. PLoS One 8, e81644 (2013).

25. Gelman, A. et al. Bayesian Data Analysis, 3rd Edition edn, 2014.

26. Glass, H.C. et al. Clinical Neonatal Seizures are Independently Associated with Outcome in Infants at Risk for Hypoxic-Ischemic Brain Injury. J Pediatr 155, 318-323 (2009).

27. Gluckman, P.D. et al. Selective head cooling with mild systemic hypothermia after neonatal encephalopathy: multicentre randomised trial. Lancet 365, 663-670 (2005).

28. Miller, S.P. et al. Seizure-associated brain injury in term newborns with perinatal asphyxia. Neurology 58, 542-548 (2002).

29. de Vries, L.S. \& Toet, M.C. Amplitude integrated electroencephalography in the full-term newborn. Clin Perinato/ 33, 619-632 (2006).

30. Fyfe, K.L., Yiallourou, S.R., Wong, F.Y. \& Horne, R.S. The development of cardiovascular and cerebral vascular control in preterm infants. Sleep Med Rev 18, 299-310 (2014).

31. Mays, R.W. et al. Development of an allogeneic adherent stem cell therapy for treatment of ischemic stroke. J Exp Stroke Transl Med 3, 34-46 (2010).

32. Lehman, N. et al. Development of a surrogate angiogenic potency assay for clinical-grade stem cell production. Cytotherapy 14, 994-1004 (2012).

33. Mallard, C. et al. Astrocytes and microglia in acute cerebral injury underlying cerebral palsy associated with preterm birth. Pediatr Res 75, 234-240 (2014).

34. Voulgari-Kokota, A. et al. Mesenchymal stem cells protect CNS neurons against glutamate excitotoxicity by inhibiting glutamate receptor expression and function. Exp Neurol 236, 161-170 (2012).

35. Volpe, J.J. Brain injury in premature infants: a complex amalgam of destructive and developmental disturbances. Lancet Neurol 8, 110-124 (2009).

36. Miron, V.E. et al. M2 microglia and macrophages drive oligodendrocyte differentiation during CNS remyelination. Nat Neurosci 16, 1211-1218 (2013).

37. Glezer, I., Simard, A.R. \& Rivest, S. Neuroprotective role of the innate immune system by microglia. Neuroscience 147, 867-883 (2007).

38. Ajmo, C.T., Jr. et al. The spleen contributes to stroke-induced neurodegeneration. J Neurosci Res 86, 2227-2234 (2008).

39. Offner, H. et al. Experimental stroke induces massive, rapid activation of the peripheral immune system. J Cereb Blood Flow Metab 26, 654-665 (2006).

40. Offner, H. et al. Splenic atrophy in experimental stroke is accompanied by increased regulatory $T$ cells and circulating macrophages. J Immunol 176, 6523-6531 (2006).

41. Seifert, H.A. et al. Pro-inflammatory interferon gamma signaling is directly associated with stroke induced neurodegeneration. Journal of Neuroimmune Pharmacology 9, 679-689 (2014).

42. Seifert, H.A. et al. The spleen contributes to stroke induced neurodegeneration through interferon gamma signaling. Metabolic brain disease 27, 131-141 (2012).

43. Liesz, A. et al. Regulatory T cells are key cerebroprotective immunomodulators in acute experimental stroke. Nat Med 15, 192-199 (2009).

44. Back, S.A., Riddle, A., Dean, J. \& Hohimer, A.R. The instrumented fetal sheep as a model of cerebral white matter injury in the premature infant. Neurotherapeutics 9, 359-370 (2012).

45. Gunn, A.J. \& Bennet, L. Fetal hypoxia insults and patterns of brain injury: insights from animal models. Clin Perinatol 36, 579-593 (2009). 


\section{Mesenchymal Stromal Cell-derived Extracellular Vesicles Protect the Fetal Brain after Hypoxia-Ischemia}

Based on:

Mesenchymal Stromal Cell-derived Extracellular Vesicles Protect the Fetal Brain after Hypoxia-Ischemia.

Daan R.M.G. Ophelders', Tim G.A.M. Wolfs', Reint K. Jellema, Alex Zwanenburg, Peter Andriessen, Tammo Delhaas, Anna-Kristin Ludwig, Stefan Radtke, Vera Peters, Leon Janssen, Bernd Giebel, Boris W. Kramer.

Stem cells translational medicine. 2016;5:754-763.

* Contributed equally 


\section{ABSTRACT}

Preterm neonates are susceptible to perinatal hypoxic-ischemic brain injury, for whom currently no treatment is available. In a preclinical animal model of hypoxic-ischemic brain injury in ovine fetuses we have previously demonstrated the neuroprotective potential of systemically administered mesenchymal stromal cells (MSCs). The mechanism of MSC treatment is unclear but suggested to be paracrine through secretion of extracellular vesicles (EVs). We therefore investigated in the current study the protective effects of mesenchymal stromal cell-derived extracellular vesicles (MSCEVs) in a preclinical model of preterm hypoxic-ischemic brain injury. Ovine fetuses were subjected to global hypoxia-ischemia through transient umbilical cord occlusion, followed by in utero intravenous administration of MSC-EVs. The therapeutic effects of MSC-EV administration were assessed by analysis of (electro-) physiological parameters and histology of the brain.

Systemic administration of MSC-EVs improved brain function by reducing the total number and duration of seizures, and by preserving baroreceptor reflex sensitivity. These functional protections were accompanied by a tendency to prevent hypomyelination. Cerebral inflammation remained unaffected by the MSC-EV treatment.

Thus, our data demonstrate that MSC-EV treatment might provide a novel strategy to reduce the neurological sequelae following hypoxic-ischemic injury of the preterm brain. Our study suggests that a cell-free preparation comprising neuroprotective MSCEVs could substitute MSCs in the treatment of preterm neonates with hypoxic-ischemic brain injury, thereby circumventing the potential risks of systemic administration of living cells. 


\section{INTRODUCTION}

Hypoxia-ischemia (HI) in the developing brain has been strongly correlated with morbidity and mortality in premature and full-term infants ${ }^{1}$. One of the induced pathologies is hypoxic ischemic encephalopathy (HIE), which is associated with adverse neurodevelopmental outcomes, resulting in enormous physical, psychological and economic burden ${ }^{1,2}$. Currently, therapeutic intervention strategies for HIE are limited. Term and late preterm neonates suffering from moderate or severe HIE are eligible for $72 \mathrm{~h}$ of therapeutic cooling, which has been shown to improve outcomes ${ }^{1,3,4}$. Preterm neonates born before 35 weeks gestational age, however, are still excluded from this therapy ${ }^{5}$. Thus, new treatment strategies are urgently warranted.

In a preclinical animal model of HIE, we have demonstrated that systemic administration of mesenchymal stromal cells (MSCs) promoted functional recovery and prevented structural injury in the preterm brain following global hypoxia-ischemia ${ }^{6}$. These effects were, in part, attributable to reduction of (neuro-) inflammatory processes coordinated by a splenic response ${ }^{6}$. However, the exact mechanisms of action of the exogenously delivered MSCs are not clear.

Initially, MSCs were considered to home into affected tissues and substitute lost cell types. Increasing evidence, however, indicates that MSCs rather exert their therapeutic effects in a paracrine manner via secretion of small extracellular vesicles (EVs) (i.e. exosomes (70-150 nm in diameter) and microvesicles (100-1000 nm) $)^{7-14}$, suggesting that administration of intact MSCs is not mandated to exert therapeutic effects. In comparison to MSCs, mesenchymal stromal cell-derived extracellular vesicles (MSC-EVs) have been shown to elicit similar biological effects after administration in various preclinical disease models, including models for kidney, cardiac and brain injury, 15-21. Recently, a therapy-refractory graft-versus-host disease patient was successfully treated with allogeneic MSC-EVs with immune suppressive effects in vitro and in vivo without showing any side effects ${ }^{22}$.

Thus, the aim of this study was to assess the therapeutic efficacy of MSC-EVs in preterm brain injury. We hypothesized that systemic administration of MSC-EVs, the paracrine mediators of MSCs, would be neuroprotective in hypoxic-ischemic injury in the preterm brain. We tested this hypothesis in a well-established preclinical animal model, in which hypoxic-ischemic brain injury was induced through transient umbilical cord occlusion (UCO) in the preterm ovine fetus ${ }^{23}$. The therapeutic effects of systemic administration of MSC-EVs on brain function were determined by evaluation of seizure burden, a measure for cortical function, and by calculating baroreflex mediated heart rate response (baroreceptor reflex sensitivity), which is needed to regulate blood pressure fluctuations, as a measurement for function of the brainstem. Structural injury was investigated by histological examination of the subcortical white matter. Anti-inflammatory effects were 
assessed with histology for microglia in the hippocampus and subcortical white matter, and detection of cerebral infiltration of T lymphocytes.

\section{MATERIALS AND METHODS}

\section{Study approval}

Experimental procedures and study design were in line with the institutional guidelines for animal experiments, and approved by the Animal Ethics Committee of Maastricht University, The Netherlands.

\section{Randomization and blinding}

24 singleton fetuses of time-mated Texel ewes were randomized in four experimental groups: (1) sham umbilical cord occlusion, saline treatment (sham-SAL $n=6$ ), (2) sham umbilical cord occlusion, MSC-EV treatment (sham-MSC-EV, $n=6$ ), (3) umbilical cord occlusion, saline treatment (HI-SAL, $n=6)$, and umbilical cord occlusion, MSC-EV treatment (HI-MSC-EV, $n=6$ ) (figure 1). The investigator performing the (sham) umbilical cord occlusions was blinded to treatment allocation. Tissue sampling and post mortem analyses were conducted in a blinded fashion. A drop-out of $16 \%(n=4)$ was observed which was primarily restricted to the sham-MSC-EV group: (i.e. 3 animals of the shamMSC-EV group and 1 animal of the HI-MSC-EV). Importantly, autopsy of these animals revealed that fetal death was exclusively caused by a single technical reason namely arterial catheter not in situ resulting in exsanguination and subsequent fetal death, thereby excluding any other cause.

\section{Animals and surgery}

Singleton fetuses were surgically instrumented at 102 days gestational age (term 147 days) as described previously ${ }^{23}$ (figure 1). In short, fetuses were exposed through a midline laparotomy of the ewe. Umbilical vessel catheters $(1.2 \mathrm{~mm}$, Covedien, Mansfield, Massachusetts, USA) were inserted in the femoral artery and brachial vein for blood pressure recordings, blood sampling, and administration of MSC-EVs. Three electrocardiogram (ECG) electrodes were attached to the fetal chest for cardiologic monitoring. Two pairs of custom-made shielded silver-tipped electroencephalogram (EEG) electrodes (Cooner wire Co., Chatsworth, CA, USA) were placed bilaterally on the dura over the parasagittal cortex. An additional reference electrode was placed in the neck $^{23}$. 
An inflatable vascular occluder (OCD16HD, 16 mm, In Vivo Metric, Healdsburg, Ca. USA) was placed around the umbilical cord for induction of transient global $\mathrm{HI}$.

An additional catheter was placed in the amniotic sac for recordings of amniotic pressure. All leads were exteriorized through a trocar hole in the maternal flank. Postoperatively, the sheep were housed individually with access to food and water ad libitum.

\section{Experimental design}

After a four day recovery period, the fetuses (106 days GA, experimental day 0) were subjected to 25 minutes of (sham) umbilical cord occlusion by rapid inflation of the vascular occluder with a predefined volume of sterile saline (figure 1). Occlusion was confirmed by an acute drop in heart rate and a gradual decline of blood pressure. Furthermore, global hypoxia-ischemia was monitored with subsequent arterial blood gas analysis (data not shown).

Animals that were randomized to receive MSC-EV treatment received 1 aliquot of $4.0 \times 10^{7}$ cell equivalents divided over two boluses of $2.0 \times 10^{7}$ cell equivalents. The first bolus of $2.0 \times 10^{7}$ cell equivalents was administered 1 hour following (sham) umbilical cord occlusion. The second bolus was administered 4 days after the hypoxic-ischemic insult. Control animals received an equal volume of sterile $0.9 \% \mathrm{NaCl}$ intravenously at the designated time points. The fetuses were sacrificed 7 days after (sham) UCO and prepared for tissue sampling.

\section{Data acquisition and analysis}

Blood pressure, amniotic pressure, ECG and EEG data were acquired and digitized by a custom-made MPAQ unit (Maastricht-Programmable AcQuisition system, Maastricht Instruments BV, Maastricht, The Netherlands) with IDEEQ software (Maastricht Instruments BV) and stored for off-line analysis, as described in detail previously ${ }^{6,23}$. Briefly, following detection and removal of high-voltage (EEG signal $>1000 \mu \mathrm{V}$ ) and flat line artefacts, raw signals were converted into amplitude-integrated EEG (aEEG) traces. The aEEG processing includes an asymmetric band-pass filter that strongly attenuates activity below $2 \mathrm{~Hz}$ and above $15 \mathrm{~Hz}$, semi-logarithmic amplitude compression and time compression. As in the neonatal intensive care unit, these aEEG traces were used to detect electrographic seizure activity, which is characterized by an abrupt rise in the lower and upper margin amplitude of the aEEG. Electrographic seizure activity with a duration $\geq 10 \mathrm{~s}$ was annotated using aEEG/EEG traces and was performed by a neonatologist, experienced in neonatal aEEG interpretation. 
To quantify baroreflex mediated heart rate response (baroreceptor reflex sensitivity, $\mathrm{ms} / \mathrm{mm} \mathrm{Hg}$ ), the ratio between the standard deviation of the mean heart rate (R-R intervals) variability and the standard deviation of the mean systolic blood pressure (SDSDP) was calculated, as reported previously ${ }^{24,25}$.

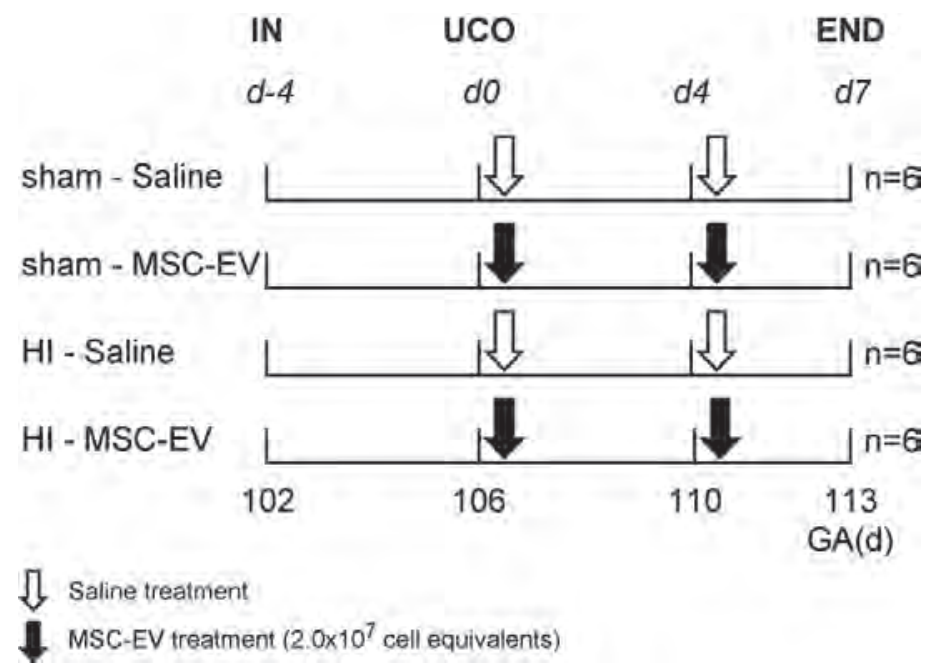

Figure 1. Study design.

Fetuses were instrumented at a gestational age of 102 days. After a recovery period of 4 days fetuses were subjected to $25 \mathrm{~min}$ of umbilical cord occlusion or sham occlusion (d0). One hour and 4 days (d4) after umbilical cord occlusion or sham occlusion, fetuses received either intravenous MSC-EVs $\left(2.0 \times 10^{7}\right.$ cell equivalents, closed arrow) or saline $0.9 \%$ (open arrow). After a 7 day reperfusion period brain tissue was collected. IN = instrumentation; UCO = umbilical cord occlusion; $E N D=$ end of experiment; $\mathrm{HI}=$ hypoxiaischemia, $\mathrm{SAL}=$ saline, $\mathrm{MSC}-\mathrm{EV}=$ mesenchymal stem cell-derived extracellular vesicles; $\mathrm{GA}(\mathrm{d})$ = gestational age (days).

MSC-EVS

Human bone marrow-derived MSCs were raised from bone marrow donors after informed consent according to the Declaration of Helsinki as described previously ${ }^{22}$. Briefly, MSCs were expanded in MSC basal media (Pan Biotech, Aidenbach, Germany) supplemented with $10 \%$ human thrombocyte lysate, $1 \%$ glutamine, and $1 \%$ penicillinstreptomycin (Invitrogen, Darmstadt, Germany). Their MSC nature was confirmed by flow cytometry with fluorescent labeled anti-CD44, anti-CD73, anti-CD90, anti-CD105 and anti-CD146 antibodies as positive markers as well as with by anti-CD14, anti-CD31 and anti-CD45 antibodies as negative controls. In addition their osteogenic and adipogenic differentiation potential was confirmed in conventional MSC differentiation assays. MSC-EVs were harvested from MSC-conditioned media which were collected from passage 3 onwards, exactly as described before. ${ }^{2}$ Briefly, media exchanges were performed every 48 hours. MSC-conditioned media were passed through a $0.22 \mu \mathrm{m}$ 
filter membrane (TPP, Trasadingen, Switzerland) and stored at $-20^{\circ} \mathrm{C}$ until usage. Upon processing all conditioned media samples where thawed and processed at once.

After adding polyethylene glycol (PEG) and $\mathrm{NaCl}$ to a final concentration of $10 \%$ PEG $6000(\mathrm{v} / \mathrm{v})$ and $75 \mathrm{mM} \mathrm{NaCl}$ over-night incubation at $4^{\circ} \mathrm{C}$, MSC-EVs were concentrated by low speed centrifugation (30 min at 1,500 g). Pellets were washed in in $0.9 \% \mathrm{NaCl}$ twice and resuspended in $0.9 \% \mathrm{NaCl}$. MSC-EVs were stored as aliquots of $1 \mathrm{~mL}$, each containing the MSC-EV equivalents harvested from the $48 \mathrm{~h}$ conditioned media of $4 \times 10^{7}$ cells. Aliquots were stored at $-80^{\circ} \mathrm{C}$ until usage. The obtained MSC-EV fraction was characterized as presented in ${ }^{22}$ by Nanoparticle Tracking Analyses (NTA) ${ }^{26}$ and Western Blot (Supplementary figure 1) as described previously. ${ }^{22}$ Briefly, protein concentrations were determined by the micro BCA assay (Thermo Fisher Scientific, Schwerte, Germany). $5 \mu \mathrm{g}$ of the concentrated MSC-EVs were treated with sample buffer (DTT, $0.1 \% \mathrm{SDS}, 0.1 \mathrm{M}$ Tris $\mathrm{HCl}, \mathrm{pH} 7.0$ ) and boiled for $5 \mathrm{~min}$ at $95^{\circ} \mathrm{C}$. Samples were separated on $12 \%$ SDS-PAGE gels and transferred to PVDF membranes (Merck Millipore, Darmstadt, Germany). Membranes were blocked in 5\% skimmed milk in PBS followed by incubation with antibodies recognizing Tsg101 (Sigma-Aldrich) or CD81 (BD). Subsequently, membranes were incubated with appropriate HRP-conjugated secondary antibodies (Dianova, Hamburg, Germany). Visualization was accomplished by enhanced chemiluminescence (Thermo Fisher Scientific). Furthermore, it was negatively tested for the presence of bacteria, viruses and endotoxins.

\section{Immunohistochemistry in the brain}

Right cerebral hemispheres were immersion fixated in $4 \%$ paraformaldehyde and subsequently embedded in gelatin. Serial coronal sections $(50 \mu \mathrm{m})$ were cut on a Leica VT $1200 S$ vibrating microtome (Leica Biosystems, Nussloch, Germany). Free-floating sections at the level of mid-thalamus and posterior hippocampus were stained for ionized calcium binding adaptor molecule (IBA-1) (Wako Pure Chemical Industries, Osaka, Japan) for microglia or for myelin basic protein (MBP) (Merck Millipore, Billerica, MA USA) for white matter, and cleaved caspase-3 for apoptosis (Cell Signaling Technology, Boston, USA) as previously described. ${ }^{23,27}$

Paraffin embedded brain coronal sections at the level of mid-thalamus and posterior hippocampus were stained for T cells with CD3 (DAKO A0452, DAKO, Denmark) as reported previously. ${ }^{6}$ Briefly, endogenous peroxidase was inactivated by incubation with $0.3 \% \mathrm{H}_{2} \mathrm{O}_{2}$, dissolved in PBS. Prior to blocking of nonspecific binding with $5 \%$ bovine serum albumin (BSA) and incubation with the primary CD3 antibody, antigen retrieval was performed in sodium citrate buffer ( $\mathrm{pH}$ 6.0) using a microwave oven. Following overnight incubation with the primary antibody, sections were incubated with the appropriate secondary biotin-labeled antibody. Immunostaining was enhanced with 
Vectastain ABC peroxidase elite kit (PK-6200, Vector Laboratories) followed by NiDAB staining and counterstaining with $0.1 \%$ nuclear fast red.

\section{Analysis immunohistochemistry}

Digital images of the hippocampus (IBA-1, 20 times magnification) and subcortical white matter (SCWM) (IBA-1, MBP, and caspase-3, 100 times magnification) were acquired on an Olympus AX-70 microscope (Olympus, Tokyo, Japan) equipped with a black-white digital camera.

Areal fractions of IBA-1 and MBP immunoreactivity, expressed as the percentage immunoreactivity of the total area using a standard threshold, were determined with Leica Qwin Pro V 3.5.1 software (Leica, Rijswijk, the Netherlands) as described previously. ${ }^{23}$

Numbers of Caspase-3 positive cells were determined with Leica Qwin Pro V 3.5.1 software, and averaged to obtain cell numbers for Caspase-3 per section.

For the analysis of CD3 staining in the SCWM, 2 coronal sections per animal were studied at the mid thalamus and posterior hippocampus level. CD3 positive cells were counted at 200 times magnification in ten fields of view on a Leica DM2000 microscope equipped with a digital camera, focused on cerebral vasculature. Averages per animal were expressed a cells per field of view.

\section{Statistics}

Animal characteristics and histological outcome parameters are shown as means with 95\% Confidence Interval $(\mathrm{Cl})$. Comparisons between groups of all parameters were drawn with analysis of variance (ANOVA) or with random intercept models in case of repeated measurements per animal (e.g. different sections per brain) as described previously. $^{28}$ Data, variables, whose distributions were positively skewed, were logtransformed for statistical testing. For interpretation purposes, averages on the log scale were transformed back to original scale and are presented as geometric means and corresponding 95\% confidence intervals. ${ }^{6}$

Seizure data, cumulative throughout the entire experiment, showed right-skewness due to the absence of seizures in non-HI groups that could not be corrected by logtransformation. Therefore, group's comparisons were performed with Mann-Whitney tests. Seizure burden is represented as median and interquartile range (IQR). P $<0.05$ was considered statistically significant. All statistical analyses have been performed with IBM SPSS Statistics version 22 (IBM Corp., Armonk, NY, USA). 
Statistical analysis of baroreceptor reflex sensitivity (BRS) was performed using a Bayesian multilevel model. ${ }^{29}$ Statistical time series analysis was conducted using the Stan for R package (version 2.6.0) in $\mathrm{R}$ (version 3.1.1).

\section{RESULTS}

\section{Animal characteristics}

To assess the neuroprotective capacities of MSC-EVs, we randomized 24 preterm ovine fetuses in different experimental groups (figure 1). Fetuses were subjected to 25 minutes of global hypoxia-ischemia (HI) through umbilical cord occlusion (UCO) or sham UCO. MSC-EVs were administered 1 hour and four days after UCO (figure 1).

Brain to bodyweight ratios did not differ significantly between the experimental groups. Splenic weight relative to bodyweight, indicative of activation of the splenic inflammatory response ${ }^{23}$ was significantly reduced after global HI (sham-SAL vs. HI-SAL; $\mathrm{P}<0.05)$, indicative of splenic involution, which is an indication of activation of the peripheral immune system. ${ }^{23,30}$ MSC-EVs prevented $\mathrm{HI}$-induced splenic involution (HISAL vs. HI-MSC-EV; P<0.05) (table 1).

Table 1. Animal characteristics.

\begin{tabular}{lllll}
\hline & sham - SAL & sham - MSC-EV & HI - SAL & HI - MSC-EV \\
\hline Brain/Bodyweight ratio & 16.77 & 21.19 & 16.19 & 16.70 \\
(g/kg BW) & $(14.80 ; 18.74)$ & $(10.48 ; 31.90)$ & $(13.50 ; 18.88)$ & $(11.27 ; 22.13)$ \\
Spleen/Bodyweight ratio & 2.68 & 3.10 & 1.72 & 2.43 \\
(g/kg BW) & $(1.82 ; 3.54)$ & $(0.25 ; 5.95)$ & $(1.45 ; 1.99)^{*}$ & $(2.03 ; 2.83)^{\#}$ \\
\hline
\end{tabular}

Brain weight corrected for bodyweight was unaffected by global HI and/or MSC-EV treatment. Splenic weight corrected for bodyweight significantly reduced following global $\mathrm{HI}$, which was significantly restored after MSCEV treatment. Ratios are depicted as means with $95 \% \mathrm{Cl}$. * $\mathrm{P} \leq 0.05$ compared to control (sham-SAL), ${ }^{\#} \mathrm{P} \leq 0.05$ compared to $\mathrm{HI}-\mathrm{SAL}$ ). $\mathrm{BW}=$ bodyweight; $\mathrm{HI}=$ hypoxia-ischemia; $\mathrm{SAL}=$ saline; $\mathrm{MSC}-\mathrm{EV}=$ mesenchymal stem cell-derived extracellular vesicles.

\section{MSC-EVs prevent loss of cortical function and baroreflex sensitivity after global HI}

Electrographic seizure burden was assessed as a measure of cortical function, and was investigated by amplitude integrated electroencephalogram (aEEG) for total numbers of seizures (figure 2A) and total amount of time of seizure activity (figure 2B). Global HI induced a profound increase in the cumulative number of seizures (sham-SAL vs. HI-SAL; $\mathrm{P}=0.003$ ) and the cumulative duration (sec.) of seizure activity (sham-SAL VS. HI-SAL; $\mathrm{P}=0.002$ ) which were reduced by MSC-EV treatment, (cumulative number: HI-SAL vs. HI- 
MSC-EV; P=0.021) (cumulative duration: HI-SAL vs. HI-MSC-EV; P=0.029). No differences in seizure burden between the sham conditions could by detected.
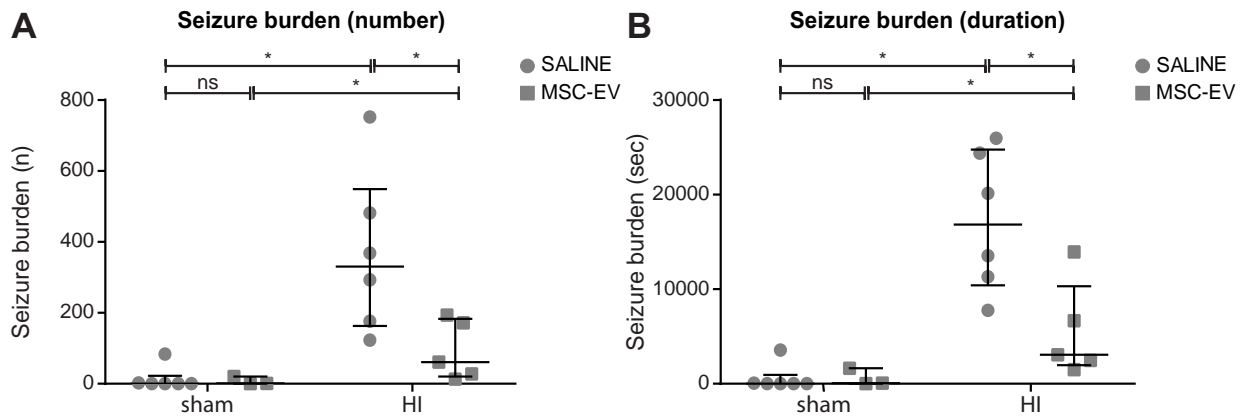

Figure 2. MSC-EV treatment induces functional neuroprotection after global $\mathrm{HI}$.

Global HI caused a significant seizure burden indicated by an increased (A) total number and (B) duration of seizures compared to sham occluded animals. Administration of MSC-EVs reduced electrographic seizure number and duration compared to saline treated animals. Medians \pm interquartile ranges and levels of significance, which were calculated by Mann-Whitney test, are depicted. ${ }^{*} \mathrm{P} \leq 0.05 ; \mathrm{NS}=$ not significant. $\mathrm{HI}=$ hypoxia-ischemia; MSC-EV = mesenchymal stem cell-derived extracellular vesicles.

Brain stem function was assessed by analysis of baroreceptor reflex sensitivity (figure 3). The baroreflex is a vital part of the vascular auto regulatory system, ensuring adequate perfusion of the fetal brain upon disturbance of homeostasis. ${ }^{24}$ Baroreceptor reflex sensitivity was reduced at experimental day 4 days following global HI when compared to controls and remained compromised throughout the experiment. MSC-EV treatment prevented $\mathrm{HI}$-induced compromise of the baroreceptor reflex sensitivity from experimental day 3 onwards. Surprisingly, MSC-EV treatment significantly reduced baroreceptor reflex sensitivity in healthy controls.

\section{MSC-EVs partially protect against HI-induced hypomyelination, but not against apoptosis}

Myelin basic protein (MBP) immunoreactivity, which was analyzed to determine white matter injury, was assessed in the subcortical white matter (SCWM) (figure 4 A-B). Global $\mathrm{HI}$ resulted in marked hypomyelination in the SCWM as indicated by a significant decrease of MBP immunoreactivity (sham-SAL vs. HI-SAL; P=0.001). MSC-EV treatment showed a mild trend to protect against hypomyelination, however statistical significance was not reached (HI-SAL vs. HI-MSC-EV; P=0.100). 


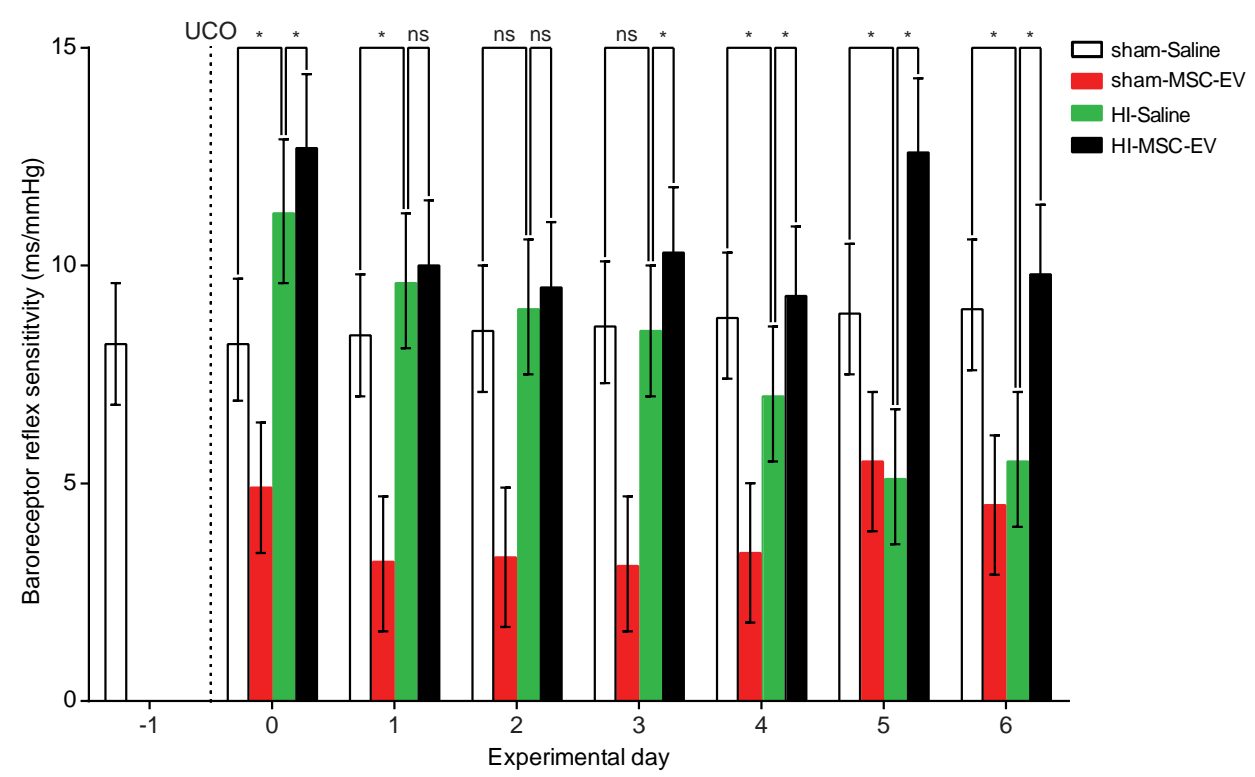

Figure 3. MSC-EVs prevented loss of baroreceptor reflex sensitivity.

Global HI (green bars) caused a significant gradual decline of baroreceptor reflex sensitivity over time, which was prevented by MSC-EV treatment (black bars); means $\pm 95 \% \mathrm{Cl}$ and levels of significance of the treatment effect (HI-SAL vs. HI-MSC-EV) are depicted, which were calculated by the Bayesian multi-level model. MSC-EV treatment significantly compromised baroreceptor reflex sensitivity in healthy controls (red bars). * $\mathrm{P} \leq 0.05$; $\mathrm{NS}=$ not significant. UCO = umbilical cord occlusion; $\mathrm{HI}$ = hypoxia-ischemia; MSC-EV= mesenchymal stromal cell-derived extracellular vesicles.

Assessment of cleaved caspase-3 in the SCWM was performed as a marker for apoptotic cell death. The numbers of caspase-3-positive cells following global HI tended to be higher at 7 days in comparison to controls $(P=0.070)$. There was no effect on the numbers of caspase -3-positive cells after hypoxia-ischemia by MSC-EV treatment (HISAL vs. HI-MSC-EV; $\mathrm{P}=0.457)$. 

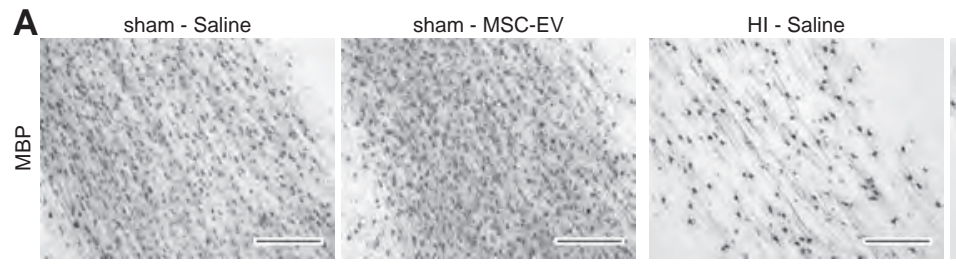

HI - MSC-EV

B

Subcortical white matter

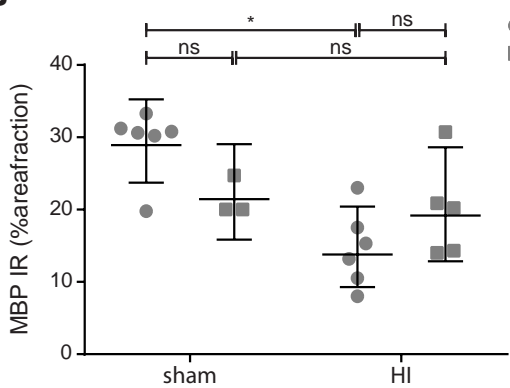

\section{Subcortical white matter}

- SALINE MSC-EV

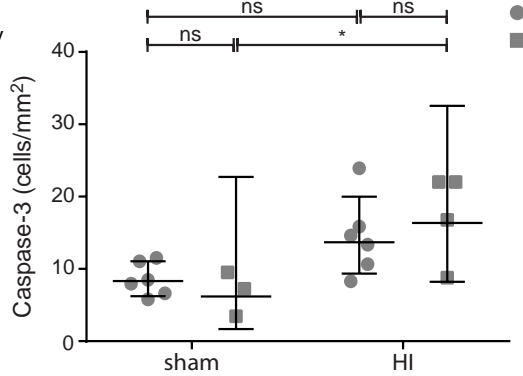

- SALINE

MSC-EV

Figure 4. MSC-EVs reduced white matter injury after global HI, but did not prevent HI-induced apoptosis.

(A) Immunohistochemical MBP staining in the subcortical white matter of all four experimental groups at 100x magnification. Global HI induced marked hypomyelination in the subcortical white matter. MSC-EVs tended to prevent the decrease in MBP reactivity after global $\mathrm{HI}$. The area fraction of MBP was similar in sham conditions. (B) Graphical representation of MBP immunoreactivity in the subcortical white matter. (C) Graphical presentation of caspase-3 cell-density in the subcortical white matter. A mild trend in increase of caspase-3 cell density was found following global HI. MSC-EV treatment did not affect apoptosis in the subcortical white matter. Levels of significance are depicted, which were calculated by the random intercept model with all repeated measures (i.e. brain sections) per animal (sham-SAL=6, sham-MSC-EV=3, HI-SAL=6, HI-MSC-EV=5). Due to positive skewing, data was log-transformed for statistical testing. For graphical presentation and interpretation, averages on the log scale were transformed to the original scale and presented as geometric means with corresponding $95 \%$ confidence intervals. ${ }^{*} \mathrm{P} \leq 0.05$; NS $=$ not significant. $\mathrm{MBP}=$ myelin basic protein $1 ; \mathrm{HI}=$ hypoxia-ischemia; $\mathrm{MSC}-\mathrm{EV}=$ mesenchymal stem cell-derived extracellular vesicles; IR = immunoreactivity. Scale bar $=200 \mu \mathrm{m}$.

\section{MSC-EVs do not protect against HI-induced neuroinflammation}

We assessed neuroinflammation in the SCWM (figure 5A-C) and the hippocampus (figure 5B-D) by analyzing immunoreactivity of ionized calcium binding adaptor protein 1 (IBA-1). Global HI induced a marked increase of IBA-1 immunoreactivity in the SCWM (sham-SAL vs. HI-SAL; $p=0.001$ ) and the hippocampus (sham-SAL vs. HI-SAL; $p<0.0001$ ), indicative of profound microglial activation and proliferation. MSC-EV treatment had no effects on hippocampal IBA-1 immunoreactivity (HI-SAL Vs. HI-MSC-EV; $P=0.398$ ), but increased IBA-1 immunoreactivity in the SCWM (HI-SAL vs. HI-MSC-EV; P=0.041). MSCEV treatment had no effects on IBA-1 immunoreactivity in both the SCWM and hippocampus in sham occluded animals compared to controls. 
A
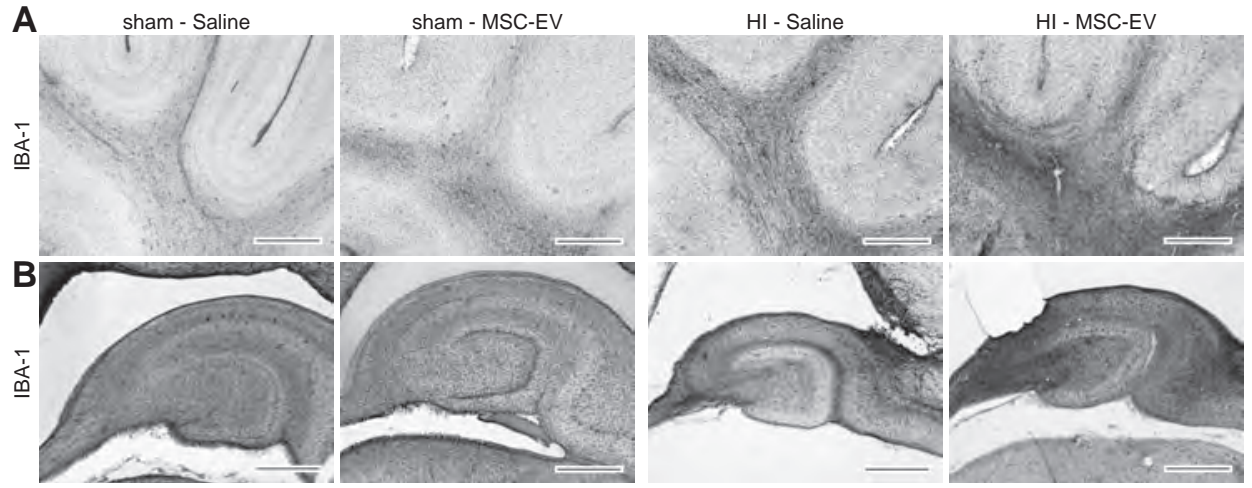

C

\section{Subcortical white matter}

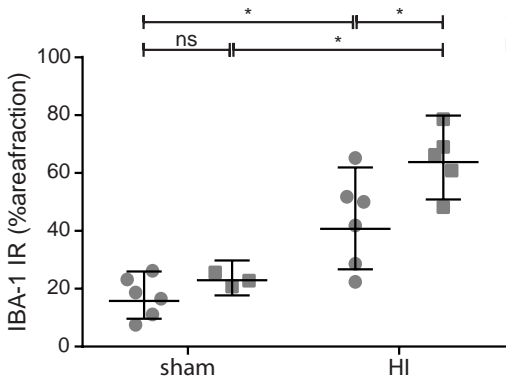

D

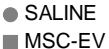

\section{Hippocampus}

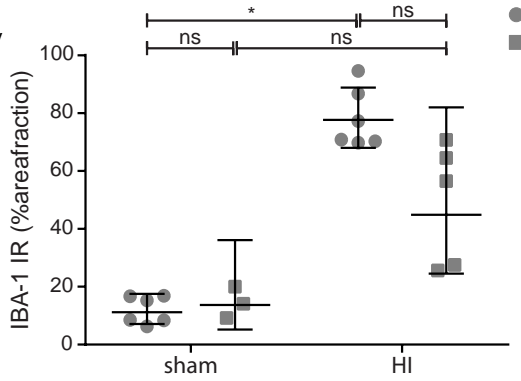

- SALINE MSC-EV

Figure 5. MSC-EVs do not reduce cerebral inflammation in the subcortical white matter and hippocampus.

(A-B) Immunohistochemical of IBA-1 staining. Global $\mathrm{HI}$ induced a significant increase of IBA-1 immunoreactivity in (A) the subcortical white matter (100x magnification) and (B) the hippocampus (20x magnification) which was not attenuated by MSC-EV treatment. (C-D) Graphical presentation of area fraction of IBA-1 immunoreactivity in (C) the subcortical white matter and (D) the hippocampus. Levels of significance are depicted, which were calculated by the random intercept model with all repeated measures (i.e. brain sections) per animal (sham-SAL=5, sham-MSC-EV=3, HI-SAL=6, HI-MSC-EV=5). Due to positive skewing, data was log-transformed for statistical testing. For graphical presentation and interpretation, averages on the log scale were transformed to the original scale and presented as geometric means with corresponding $95 \%$ confidence intervals. * P $\leq 0.05$; NS = not significant. IBA-1 = ionized calcium binding adaptor molecule $1 ; \mathrm{HI}=$ hypoxia-ischemia; MSC-EV = mesenchymal stem cell-derived extracellular vesicles, IR = immunoreactivity. Scale bar $=1000 \mu \mathrm{m}$.

Finally, we assessed the influx of T lymphocytes in the fetal brain by immunohistochemical analysis for CD3 in the SCWM (figure 6). Cerebral influx of CD3 positive cells tended to increase following global HI (sham-SAL vs. HI-SAL; p=0.078). Remarkably, MSC-EV treatment resulted in an increase of CD3 positive cells in the SCWM of sham occluded animals (sham-SAL vs. sham-MSC-EV; $\mathrm{P}=0.013$ ). 
A
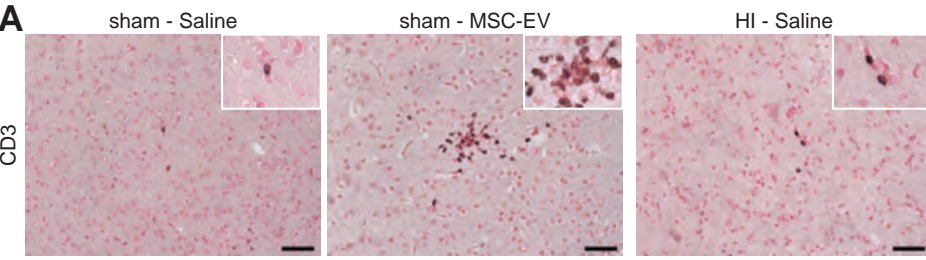

HI - MSC-EV

B

Subcortical white matter

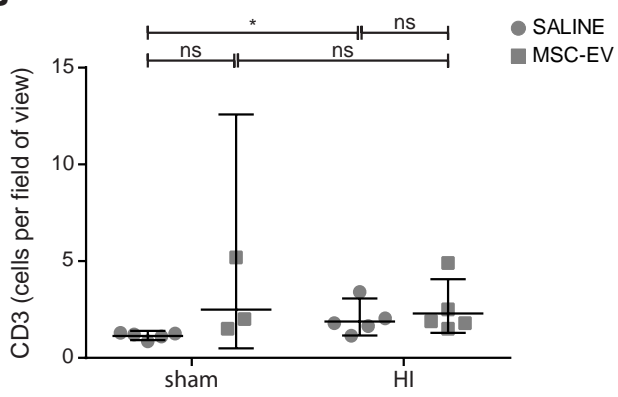

Figure 6. MSC-EVs increase cerebral influx of T-effector cells.

(A) Immunohistochemical staining for CD3 positive $T$ lymphocytes in the subcortical white matter of all four experimental groups at 200x magnification (insert 400x magnification). (B) Graphical representation of CD3 positive cells per field of view in the subcortical white matter. MSC-EV treatment increased numbers of CD3 positive T-lymphocytes in the subcortical white matter compared to saline treated animals. Levels of significance are depicted, which were calculated by the random intercept model with all repeated measures (i.e. brain sections) per animal (sham-SAL=5, sham-MSC-EV=3, HI-SAL=5, HI-MSC-EV=5). Due to positive skewing, data was log-transformed for statistical testing. For graphical presentation and interpretation, averages on the log scale were transformed to the original scale and presented as geometric means with corresponding $95 \%$ confidence intervals. * $\mathrm{P} \leq 0.05 ; \mathrm{NS}=$ not significant. $\mathrm{HI}=$ hypoxia-ischemia; $\mathrm{MSC}-\mathrm{EV}=$ mesenchymal stem cell-derived extracellular vesicles. Scale bar $=100 \mu \mathrm{m}$.

\section{DISCUSSION}

In the current study we showed that intravenous administration of MSC-EVs prevented functional impairment and showed a tendency to protect against structural injury of the preterm brain after global $\mathrm{HI}$ apparently without reducing cerebral inflammation. These findings are clinically highly relevant since post-ischemic seizure activity is associated with adverse neurodevelopmental outcomes. ${ }^{31-34}$ The baroreceptor reflex buffers shortterm changes in blood pressure by adapting systemic vascular resistance, myocardial contractility and heart rate. The clinical relevance of an impaired baroreceptor reflex function relates to the increased risk for developing additional brain injury by exposing the vulnerable developing cerebral vascular network to (large) fluctuations in blood pressure. In preterm infants, fluctuations associated with high blood pressure may disrupt the cerebral capillaries in the germinal matrix while periods of low blood pressure may cause localized hypoxia-ischemia of the watershed areas. ${ }^{35}$ Moreover, preterm infants display fluctuating pressure-passivity between systemic blood pressure 
and cerebral blood flow representing a considerably increased risk of cerebral hemorrhage or hypoxia. ${ }^{36}$ Previously, we have shown that $\mathrm{HI}$ reduces the baroreceptor reflex mediated heart rate response. ${ }^{24}$ In this paper, we demonstrate for the first time that MSC-EV treatment results in preservation of baroreceptor reflex sensitivity after exposure to global HI. Our data suggest a wide-spread functional protection by MSC-EVS of the central nervous system after global HI. Remarkably, we found that MSC-EV treatment reduced baroreceptor reflex sensitivity in healthy controls suggesting that MSC-EV treatment should be given with care in order to avoid adverse effects, and warranting further analyses of the effects of MSC-EVs on the developing brain in preclinical animal models before introducing MSC-EVs into clinical trials. However, clinical data in an adult GVHD patient demonstrated safe intravenous administration of MSC-EVs without adverse cardiovascular and hemodynamic outcomes. ${ }^{22}$

Consistent with improved electro cortical function and preservation of baroreceptor reflex sensitivity, we demonstrated that intravenous administration of MSC-EVs tended to protect against $\mathrm{HI}$-induced white matter injury, which is the clinical hallmark of neonatal $\mathrm{HI}$ brain injury. ${ }^{37}$ This study suggests that the functional neuroprotective effects of MSC-EVs are not primarily caused by anti-inflammatory mechanisms. This is in contrast to our previous results in which the neuroprotective effects of intravenously administered MSCs could be, at least in part, attributed to anti-inflammatory capacities of $\mathrm{MSCs}^{6}$. Although prevention of splenic involution ${ }^{6}$, might indicate inhibition of systemic immune activation ${ }^{30}$, this effect was not paralleled by anti-inflammatory modifications of the ischemic fetal brain. This latter finding is in contrast with results from our previous study where splenic involution was associated with prevention of cerebral inflammation after systemic MSC administration. This difference in antiinflammatory properties of MSCs-EVs in the current and MSC in the previous study could be explained by two reasons: First, the immune modulatory properties of MSCS and their EVs differ between independent MSC preparations. ${ }^{22}$ Second, in contrast to MSC-EVs, MSCs can sense micro environmental conditions to which they are exposed (e.g. licensing); a pro-inflammatory environment MSCs stimulates polarization towards an anti-inflammatory phenotype, whereas alternative stimulation has been reported to rather propagate a pro-inflammatory phenotype. ${ }^{38-45}$ This may affect their secretion of therapeutically active immune modulatory components. However, recently we compared the therapeutic effect of MSC-EVs and that of corresponding MSCs in a murine ischemic stroke model and did not recognize any differences. ${ }^{21}$ Although, such a side by side comparison was not performed in our model, this latter study suggests that differences between the two studies cannot be attributed to licensing.

The concept of inflammation-independent effects as neuroprotective mechanisms of MSC-EVs therapy is supported by several studies showing that MSC-EVs can prevent apoptosis and stimulate angiogenesis after hypoxia-ischemia. ${ }^{16,} 20,46-50$ However, no effects on angiogenic markers (mRNA levels of vascular endothelial growth factor 
(VEGF) A and VEGF-receptor 2; data not shown) or on the number of apoptotic cells after global HI with MSC-EV treatment were found in our study, suggesting that the neuroprotective potential of MSC-EV cannot be attributed to anti-apoptotic or angiogenic properties.

Despite the identified discrepancies in therapeutic effects between MSCs and MSC-EVs used in the current study, the application of EVs offers several advantages over the administration of MSCs: (1) the risk of malignant transformation is greatly reduced because EVs are non-self-replicating. ${ }^{51}$ (2) Lacking an own metabolism, the EVs activity can hardly be influenced by the in vivo environment in patients, thus allowing for a much better characterization of their functional properties. (3) Owing to their small size EVs are less likely to generate emboli upon intravenous administration, as may be the case with MSCs. (4) In addition, EVs can be sterilized by filtration. Thus, from a regulatory point of view, the production and especially the quality control of EV fractions for clinical treatment application is less complicated than for a cellular therapeutic of in vitro expanded cells. ${ }^{52}$ (5) Last but not least, EVs can be developed independently of the original donor as they are derived from MSCs from unrelated donors and thus offer the opportunity to be turned into an "off-the-shelf" product.

We have chosen this well-established preclinical sheep model since it enables us to accurately mimic the etiology of hypoxic-ischemic injury of the developing preterm brain, including continuous registration of clinical parameters with strong clinical relevance and predictive values. ${ }^{6}, 53,54$ Nevertheless, our study has several shortcomings. We have no information on the dynamics of the effects of MSC-EVs nor have we studied different doses. Time course information is thus desirable in our model. In future experiments, back-to-back comparison of MSCs and their corresponding EV fraction will provide crucial mechanistic insights into the mode of action of MSCs and their EVs. The scope of the current study was however assessing the feasibility of MSC-EV treatment to improve brain function after hypoxia-ischemia.

In conclusion, we have demonstrated in a preclinical animal model that MSC-EVs harbor neuroprotective potential as shown by improved functional and structural outcomes of

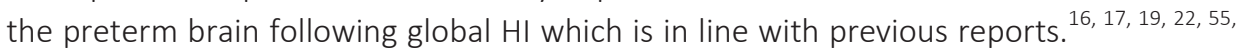
${ }^{56}$ Future studies to determine the optimal EV-contents and dosing strategy should be performed in order to establish a clinically safe, cell-free therapy for preterm babies after hypoxia-ischemia. 


\section{SUPPLEMENTARY FIGURES}

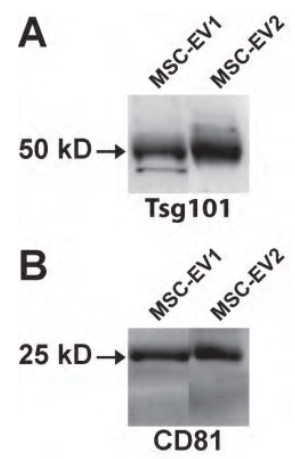

Supplementary figure 1. Characterization of MSC-EV fractions with Western blot.

MSC-EV1 was the MSC-EV fraction used in the successful treatment of a GVHD patient ${ }^{22}$ and was used as a positive control for the MSC-EV fraction used in the current study (labeled as MSC-EV2 in this figure). Obtained MSC-EV fractions were positive for exosomal markers Tsg101 (A) and CD81 (B). 


\section{REFERENCES}

1. Fleiss, B. et al. Inflammation-induced sensitization of the brain in term infants. Developmental Medicine \& Child Neurology 57, 17-28 (2015).

2. Beck, S. et al. The worldwide incidence of preterm birth: a systematic review of maternal mortality and morbidity. Bull World Health Organ 88, 31-38 (2010).

3. Jacobs, S.E. et al. Cooling for newborns with hypoxic ischaemic encephalopathy. Cochrane Database Syst Rev 1, CD003311 (2013).

4. Azzopardi, D. et al. Pilot study of treatment with whole body hypothermia for neonatal encephalopathy. Pediatrics 106, 684-694 (2000).

5. Briatore, E. et al. EEG findings in cooled asphyxiated newborns and correlation with site and severity of brain damage. Brain Dev 35, 420-426 (2013).

6. Jellema, R.K. et al. Mesenchymal stem cells induce T-cell tolerance and protect the preterm brain after global hypoxia-ischemia. PLoS One 8, e73031 (2013).

7. Lee, R.H. et al. Intravenous hMSCs improve myocardial infarction in mice because cells embolized in lung are activated to secrete the anti-inflammatory protein TSG-6. Cell Stem Cell 5, 54-63 (2009).

8. Zanotti, L. et al. Encapsulated mesenchymal stem cells for in vivo immunomodulation. Leukemia 27, 500503 (2013).

9. Lai, R.C. et al. Exosome secreted by MSC reduces myocardial ischemia/reperfusion injury. Stem Cell Res 4, 214-222 (2010).

10. Timmers, L. et al. Reduction of myocardial infarct size by human mesenchymal stem cell conditioned medium. Stem cell research 1, 129-137 (2007).

11. Gnecchi, M. et al. Paracrine action accounts for marked protection of ischemic heart by Akt-modified mesenchymal stem cells. Nat Med 11, 367-368 (2005).

12. Gnecchi, M. et al. Evidence supporting paracrine hypothesis for Akt-modified mesenchymal stem cellmediated cardiac protection and functional improvement. Faseb J 20, 661-669 (2006).

13. Katsuda, T., Kosaka, N., Takeshita, F. \& Ochiya, T. The therapeutic potential of mesenchymal stem cellderived extracellular vesicles. Proteomics 13, 1637-1653 (2013).

14. Fleiss, B. et al. Stem cell therapy for neonatal brain injury. Clinics in perinatology 41, 133-148 (2014).

15. Bruno, S. et al. Mesenchymal stem cell-derived microvesicles protect against acute tubular injury. J Am Soc Nephrol 20, 1053-1067 (2009).

16. Xin, H. et al. Systemic administration of exosomes released from mesenchymal stromal cells promote functional recovery and neurovascular plasticity after stroke in rats. J Cereb Blood Flow Metab 33, 17111715 (2013).

17. Gatti, S. et al. Microvesicles derived from human adult mesenchymal stem cells protect against ischaemia-reperfusion-induced acute and chronic kidney injury. Nephrol Dial Transplant 26, 1474-1483 (2011).

18. Bruno, S. et al. Microvesicles derived from mesenchymal stem cells enhance survival in a lethal model of acute kidney injury. PLoS One 7, e33115 (2012).

19. Lai, R.C., Chen, T.S. \& Lim, S.K. Mesenchymal stem cell exosome: a novel stem cell-based therapy for cardiovascular disease. Regen Med 6, 481-492 (2011).

20. Zhang, Y. et al. Effect of exosomes derived from multipluripotent mesenchymal stromal cells on functional recovery and neurovascular plasticity in rats after traumatic brain injury. Journal of neurosurgery 122, 856-867 (2015).

21. Doeppner, T.R. et al. Extracellular Vesicles Improve Post-Stroke Neuroregeneration and Prevent Postischemic Immunosuppression. Stem Cells Transl Med 4, 1131-1143 (2015).

22. Kordelas, L. et al. MSC-derived exosomes: a novel tool to treat therapy-refractory graft-versus-host disease. Leukemia 28, 970-973 (2014).

23. Jellema, R.K. et al. Cerebral inflammation and mobilization of the peripheral immune system following global hypoxia-ischemia in preterm sheep. Journal of neuroinflammation 10, 13 (2013). 
24. Zwanenburg, A. et al. Heart rate-mediated blood pressure control in preterm fetal sheep under normal and hypoxic-ischemic conditions. Pediatr Res 73, 420-426 (2013).

25. Andriessen, P. et al. Cardiovascular fluctuations and transfer function analysis in stable preterm infants. Pediatr Res 53, 89-97 (2003).

26. Sokolova, V. et al. Characterisation of exosomes derived from human cells by nanoparticle tracking analysis and scanning electron microscopy. Colloids Surf B Biointerfaces 87, 146-150 (2011).

27. Kuypers, E. et al. Effects of intra-amniotic lipopolysaccharide and maternal betamethasone on brain inflammation in fetal sheep. PLoS One 8, e81644. (2013).

28. Jellema, R.K. et al. Systemic G-CSF attenuates cerebral inflammation and hypomyelination but does not reduce seizure burden in preterm sheep exposed to global hypoxia-ischemia. Experimental neurology 250, 293-303 (2013).

29. Gelman, A. et al. Bayesian Data Analysis, 3rd Edition edn, 2014.

30. Ajmo, C.T., Jr. et al. The spleen contributes to stroke-induced neurodegeneration. J Neurosci Res 86 , 2227-2234 (2008).

31. Gluckman, P.D. et al. Selective head cooling with mild systemic hypothermia after neonatal encephalopathy: multicentre randomised trial. Lancet 365, 663-670 (2005).

32. Glass, H.C. et al. Clinical Neonatal Seizures are Independently Associated with Outcome in Infants at Risk for Hypoxic-Ischemic Brain Injury. J Pediatr 155, 318-323 (2009).

33. Miller, S.P. et al. Seizure-associated brain injury in term newborns with perinatal asphyxia. Neurology 58, 542-548 (2002).

34. de Vries, L.S. \& Toet, M.C. Amplitude integrated electroencephalography in the full-term newborn. Clin Perinatol 33, 619-632, vi (2006).

35. Perlman, J.M. The relationship between systemic hemodynamic perturbations and periventricularintraventricular hemorrhage-A historical perspective. Seminars in pediatric neurology; 2009: Elsevier; 2009. p. 191-199.

36. Fyfe, K.L., Yiallourou, S.R., Wong, F.Y. \& Horne, R.S. The development of cardiovascular and cerebral vascular control in preterm infants. Sleep medicine reviews (2013).

37. Volpe, J.J. Brain injury in premature infants: a complex amalgam of destructive and developmental disturbances. The Lancet Neurology 8, 110-124 (2009).

38. Dazzi, F., Lopes, L. \& Weng, L. Mesenchymal stromal cells: a key player in 'innate tolerance'? Immunology 137, 206-213 (2012).

39. Waterman, R.S., Tomchuck, S.L., Henkle, S.L. \& Betancourt, A.M. A new mesenchymal stem cell (MSC) paradigm: polarization into a pro-inflammatory MSC1 or an Immunosuppressive MSC2 phenotype. PLoS One 5, e10088 (2010).

40. Bernardo, M.E. \& Fibbe, W.E. Mesenchymal stromal cells: sensors and switchers of inflammation. Cell stem cell 13, 392-402 (2013).

41. Li, W. et al. Mesenchymal stem cells: a double-edged sword in regulating immune responses. Cell Death Differ 19, 1505-1513 (2012).

42. Hemeda, H. et al. Interferon-gamma and tumor necrosis factor-alpha differentially affect cytokine expression and migration properties of mesenchymal stem cells. Stem Cells Dev 19, 693-706 (2010).

43. Ranganath, S.H., Levy, O., Inamdar, M.S. \& Karp, J.M. Harnessing the mesenchymal stem cell secretome for the treatment of cardiovascular disease. Cell stem cell 10, 244-258 (2012).

44. Prockop, D.J. Repair of tissues by adult stem/progenitor cells (MSCs): controversies, myths, and changing paradigms. Molecular Therapy 17, 939-946 (2009).

45. Gregory, C.A., Ylostalo, J. \& Prockop, D.J. Adult bone marrow stem/progenitor cells (MSCs) are preconditioned by microenvironmental" niches" in culture: a two-stage hypothesis for regulation of MSC fate. Science Signaling 2005, pe37-pe37 (2005).

46. Salomon, C. et al. Exosomal signaling during hypoxia mediates microvascular endothelial cell migration and vasculogenesis. PLoS One 8, e68451 (2013).

47. Bian, S. et al. Extracellular vesicles derived from human bone marrow mesenchymal stem cells promote angiogenesis in a rat myocardial infarction model. J Mol Med (Berl) 92, 387-397 (2014). 


\section{Chapter 3}

48. Zhang, B. et al. Human umbilical cord mesenchymal stem cell exosomes enhance angiogenesis through the Wnt4/beta-catenin pathway. Stem Cells Transl Med 4, 513-522 (2015).

49. Chen, J. et al. Proangiogenic compositions of microvesicles derived from human umbilical cord mesenchymal stem cells. PLoS One 9, e115316 (2014).

50. Doeppner, T.R. et al. Extracellular Vesicles Improve Post-Stroke Neuroregeneration and Prevent Postischemic Immunosuppression. Stem cells translational medicine 4, 1131-1143 (2015).

51. Dlouhy, B.J., Awe, O., Rao, R.C., Kirby, P.A. \& Hitchon, P.W. Autograft-derived spinal cord mass following olfactory mucosal cell transplantation in a spinal cord injury patient: Case report. Journal of Neurosurgery: Spine 21, 618-622 (2014).

52. Lener, T. et al. Applying extracellular vesicles based therapeutics in clinical trials-an ISEV position paper. Journal of extracellular vesicles 4 (2015).

53. Back, S.A., Riddle, A., Dean, J. \& Hohimer, A.R. The instrumented fetal sheep as a model of cerebral white matter injury in the premature infant. Neurotherapeutics 9, 359-370 (2012).

54. Gunn, A.J. \& Bennet, L. Fetal hypoxia insults and patterns of brain injury: insights from animal models. Clin Perinatol 36, 579-593 (2009).

55. Xin, H. et al. Exosome-mediated transfer of miR-133b from multipotent mesenchymal stromal cells to neural cells contributes to neurite outgrowth. Stem Cells 30, 1556-1564 (2012).

56. Li, T. et al. Exosomes derived from human umbilical cord mesenchymal stem cells alleviate liver fibrosis. Stem Cells Dev 22, 845-854 (2013). 


\section{4}

\section{Neuroinflammation and Structural Injury of the Fetal Ovine Brain Following Intra- amniotic Candida albicans Exposure}

Based on:

Neuroinflammation and Structural Injury of the Fetal Ovine Brain Following Intra-amniotic Candida albicans Exposure.

Daan R.M.G. Ophelders*, Ruth Gussenhoven*, Martin Lammens, Benno Küsters, Matthwe W. Kemp, John P. Newnham, Matthew S. Payne, Suhas G. Kallapur, Allan H. Jobe, Luc Zimmermann, Boris W. Kramer, Tim G.A.M. Wolfs.

Journal of Neuroinflammation. 2016;13(1):1.

* Contributed equally 


\section{ABSTRACT}

Intra-amniotic Candida albicans infection is associated with preterm birth and high morbidity and mortality rates. Survivors are prone to adverse neurodevelopmental outcomes. The mechanisms leading to these adverse neonatal brain outcomes remain largely unknown. To better understand the mechanisms underlying Candida albicansinduced fetal brain injury, we studied immunological responses and structural changes of the fetal brain in a well-established translational ovine model of intra-amniotic Candida albicans infection. In addition, we tested whether these potential adverse outcomes of the fetal brain were improved in utero by antifungal treatment with Fluconazole.

Pregnant ewes received an intra-amniotic injection of $10^{7}$ colony forming units Candida albicans or saline (controls) at 3 or 5 days before preterm delivery at 0.8 of gestation (term 147 days). Fetal intra-amniotic/intra-peritoneal injections of Fluconazole or saline (controls) were administered 2 days after Candida albicans exposure. Post mortem analyses for fungal burden, peripheral immune activation, neuroinflammation and white matter/ neuronal injury were performed to determine the effects of intraamniotic Candida albicans and Fluconazole treatment.

Intra-amniotic exposure to Candida albicans caused a severe systemic inflammatory response, illustrated by a robust increase of plasma IL- 6 concentrations. Cerebrospinal fluid cultures were positive for Candida albicans in the majority of the 3 day Candida albicans-exposed animals whereas no positive cultures were present in the 5 day Candida albicans-exposed and Fluconazole treated animals. Although Candida albicans was not detected in the brain parenchyma, a neuroinflammatory response in the hippocampus and white matter was seen which was characterized by increased microglial and astrocyte activation. These neuroinflammatory changes were accompanied by structural white matter injury. Intra-amniotic Fluconazole reduced fetal mortality, but did not attenuate neuroinflammation and white matter injury.

Intra-amniotic Candida albicans exposure provoked acute systemic and neuroinflammatory responses with concomitant white matter injury. Fluconazole treatment prevented systemic inflammation without attenuating cerebral inflammation and injury. 


\section{INTRODUCTION}

Preterm birth is associated with chorioamnionitis which is defined as inflammation of the fetal membranes and amniotic fluid caused by microbial invasion. ${ }^{1,2}$ The microorganisms most frequently associated with this condition include Ureaplasma species, Mycoplasma hominis, and Gardnerella vaginalis all of which most commonly originate from the lower reproductive tract. ${ }^{3}$ These microorganisms and/or inflammatory mediators in the amniotic cavity can cause a fetal inflammatory response syndrome (FIRS)..$^{4-6}$ Chorioamnionitis and subsequent FIRS are independent risk factors for adverse outcomes, including injury of the fetal brain., 7 Adverse neurodevelopmental outcomes result from diffuse cerebral inflammation and white matter injury, periventricular leukomalacia and intraventricular hemorrhage. ${ }^{5,8,9}$ These conditions are associated with a high mortality rate and survivors are predisposed to long-term morbidity including mental retardation, impaired learning and visual disorders and in severe cases cerebral palsy. $7,8,10$

The pathophysiology of chorioamnionitis can also include viral and fungal species. ${ }^{4,11,12}$ Candida albicans (C. albicans) is a commensal fungus of the gastro-intestinal tract which can be asymptomatic in the vaginal microbiota with increasing incidence during pregnancy. ${ }^{13,14}$ Intra-amniotic C. albicans infections are associated with high mortality rates and severely impaired neurodevelopmental outcomes ${ }^{13-16}$ in which the mechanisms linking fetal exposure to neurological pathologies remain essentially unstudied. We hypothesized that antenatal exposure to C. albicans caused a neuroinflammatory response and subsequent white matter injury, which we tested by exposing fetal sheep to intra-amniotic C. albicans. ${ }^{16,17}$

In two clinical cases intra-amniotic $C$. albicans infections resolved after oral and intraamniotic Fluconazole. ${ }^{13}$ Fluconazole is the most frequently used antifungal in C. albicans infections, and inhibits ergosterol synthesis, which is an essential component of fungal cell membranes. ${ }^{18}$ We therefore further hypothesized that fetal intra-amniotic and intra-peritoneal administration of Fluconazole would reduce the neuroinflammatory response and subsequent white matter injury to the fetal brain. Accordingly, systemic immune activation, neuroinflammation and structural white matter injury were assessed in the fetal sheep exposed to intra-amniotic $C$. albicans and treated with Fluconazole. 


\section{METHODS}

\section{Experimental design}

The study was approved by and performed according to the guidelines of the animal ethics committee of the University of Western Australia (Perth, Australia). Twenty-six date-mated Merino ewes with singleton pregnancies were randomly assigned to receive an intra-amniotic injection of saline $(2 \mathrm{~mL})$ as a control or C. albicans ( $10^{7}$ colony forming units (CFU), Western Australian clinical isolate). ${ }^{16}$ After 2 days an intraamniotic/-peritoneal injection of Fluconazole (F) (30 mg per injection, Claris Life Sciences Limited, Chacharwadi-vasana, Ahmedabad-382 213, India) or saline (controls) was administered with delivery after 1 and 3 days (Figure 1). Intra-amniotic injections were performed as previously described. ${ }^{17}$

We did not include a 5D C. albicans only group since previous results with this model indicated that 5 days exposure to C. albicans alone was lethal. ${ }^{16,17}$ Given the survival of 5D C. albicans/Fluconazole-treated group, we have concluded that Fluconazole in this model increases survival. ${ }^{16,17}$

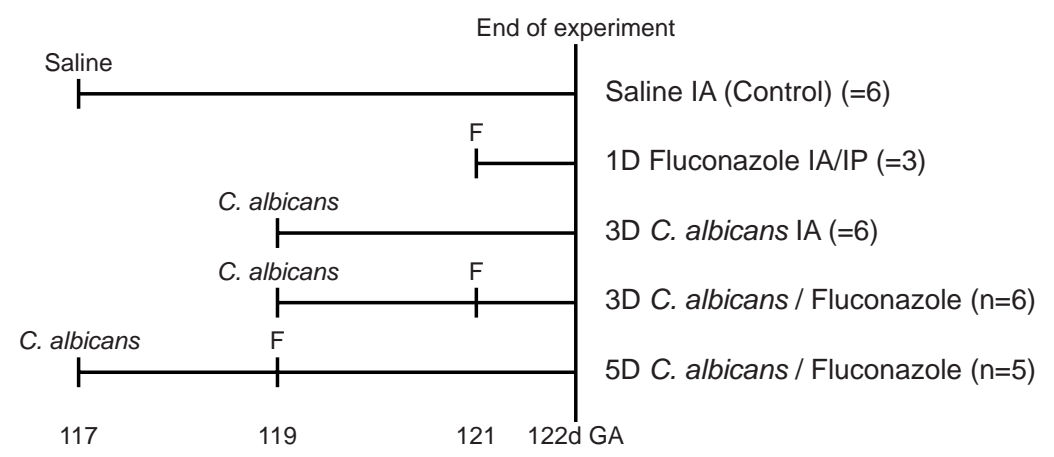

Figure 1 Experimental setup.

Animals were exposed to C. albicans for 3 or 5 days in the absence or presence of intra-amniotic (IA) / intraperitoneal (IP) Fluconazole (F). Control animals received intra-amniotic (IA) injection with saline. Fetuses were delivered at 0.8 of gestation.

\section{Tissue collection}

Fetuses were delivered by Caesarean section at 0.8 of gestation (term $\sim 147$ days) ${ }^{19}$, equivalent of 32 - 34 weeks human gestation ${ }^{20}$, and euthanized with intravenous pentobarbitone (100 mg/kg). Amniotic fluid, blood and cerebrospinal fluid (CSF) were collected at delivery and cultures for C. albicans were performed. ${ }^{16}$ Briefly, amniotic fluid $(100 \mu \mathrm{L})$ was inoculated onto Difco Sabaraud-Dextrose agar and incubated at $37{ }^{\circ} \mathrm{C}$ for $48 \mathrm{~h}$. Blood $(2 \mathrm{~mL})$ and liquor $(1 \mathrm{~mL})$ was inoculated into BACTEC Peds Plus culture 
vials (Becton Dickinson, Frankln Lakes, NJ) and incubated aerobically at $37{ }^{\circ} \mathrm{C}$ for 72 hours. ${ }^{16}$ Brains were immersion fixated in $4 \%$ paraformaldehyde.

Enzyme-linked immunosorbent assay IL-6.

Plasma interleukin (IL)-6 concentrations were assessed as an indication for systemic inflammation using a sheep-specific sandwich enzyme-linked immunosorbent assay (ELISA). First, 96 well plates were pre-coated with $100 \mu \mathrm{L}$ of mouse-anti-ovine monoclonal antibody (MAB1004, Millipore, Darmstadt, Germany, working concentration $1: 200)$ overnight at $4^{\circ} \mathrm{C}$. Plates were washed three times with $0.05 \%$ tween-20 in phosphate buffered saline (PBST) Non-specific binding was blocked with a blocking buffer (1\% bovine serum albumin (BSA) in PBST) for 1 hour followed by a washing step.

Protein standards were prepared by serial dilution of recombinant IL-6 (ImmunoChemistry Technologies, Bloomington, MN, USA). Plasma samples (100 $\mu \mathrm{L}$ ) were loaded per well in duplicate and incubated for 2 hours at room temperature. Unbound protein was omitted by washing with PBST, followed by incubation with detection antibody (rabbit-anti-ovine IL-6, AB1839, Millipore, Darmstadt, Germany, working concentration 1:500) for 60 minutes and subsequent washing. Next, each well was incubated for 30 minutes with $100 \mu \mathrm{L}$ of a goat-anti-rabbit-HRP (Jackson ImmunoResearch Laboratories Inc, West Grove, PA, USA, working concentration 1:500) followed by washing and incubation with 3,3',5,5'-tetramethylbenzidine (TMB) substrate solution for 15 minutes at room temperature. The reaction was stopped by addition of $50 \mu \mathrm{L} 2 \mathrm{~N}$ sulfuric acid to each well. Plates were then read on an ELISA plate reader at $450 \mathrm{~nm}$.

\section{Histology and immunohistochemistry.}

The right hemisphere was divided into 4 defined anatomical regions. Serial coronal sections of identical thickness $(4 \mu \mathrm{m})$ of the region containing the posterior hippocampus and mid-thalamus were cut with a Leica RM2235 microtome. Within this region, we analyzed hippocampus and the cerebral white matter since there regions are most affected following intra-uterine infection at this developmental stage. ${ }^{21}$ All stainings were performed in series (every $15^{\text {th }}$ section) and four sections per staining per animal were analyzed. Hematoxylin and eosin (H\&E) and periodic acid-Schiff (PAS) stainings were performed for morphological and anatomical analysis and for identification of pseudohyphae of $C$. albicans, respectively. Adjacent sections were stained for ionized calcium binding adaptor molecule 1 (IBA-1) (Wako Pure Chemical Industries, Osaka, Japan) for microglia, glial fibrillary acidic protein (GFAP) (DAKO Z0334) for astrocytes, myelin basic protein (MBP) (Merck Millipore, MAB386) for myelin 
sheaths, 2',3'-Cyclic-nucleotide 3'-phosphodiesterase (CNPase) (Sigma, C5922) for mature myelin producing oligodendrocytes, oligodendrocyte transcription factor 2 (Olig2) (Millipore, AB9610) for oligodendrocyte lineage cells, cluster of differentiation (CD) 68 (DAKO, M0718) for active macrophages, myeloperoxidase (MPO) (DAKO, A0398) for neutrophils, CD3 (DAKO A0452) for T-lymphocytes, Ki67 (DAKO, M7240) for cell proliferation, and cleaved caspase-3 (Cell signalling, \#9661) for apoptosis.

Sections were deparaffinized and rehydrated. Endogenous peroxidase activity was inactivated with $0.3 \% \mathrm{H}_{2} \mathrm{O}_{2}$ treatment. For every immunolabeled stain with the exception of GFAP and CD68, antigen retrieval was performed by microwave boiling of tissue sections in citrate buffer, $\mathrm{pH}$ 6.0. For CD68, antigen retrieval was performed with proteinase $\mathrm{K}$ (DAKO, S3004) treatment for 5 minutes at $37^{\circ} \mathrm{C}$. Nonspecific binding was blocked by incubation with 5\% BSA (MBP, Olig2 and CD3) or goat serum in PBS (IBA-1, GFAP, CNPase, CD68, MPO and Ki67). Sections were incubated overnight at $4^{\circ} \mathrm{C}$ in a closed humidity chamber with primary antibody (IBA-1, GFAP, MBP, CNPase and caspase-3 1:1000; Olig2, MPO and CD3 1:200; Ki67 1:100; CD68 1:50), and subsequently incubated with the specific secondary antibody. Immunostaining was enhanced with a Vectastain ABC peroxidase Elite kit (Vector Laboratories Inc, Burlingame, CA) followed by a (nickel) 3,3'-diaminobenzidine (DAB) staining and 0.1\% Nuclear Fast Red (Olig2, CD3, Ki67 and caspase-3) or Mayer's Hematoxylin (CD68 and MPO) for background staining. Sections were dehydrated and coverslipped.

To identify the mature and total number of oligodendrocytes, an immunohistochemical procedure for sequential double staining of CNPase and Olig2 was applied. In this procedure, sections were treated as described above and incubated with the antiCNPase antibody, followed by incubation with a biotinylated anti-mouse secondary antibody and visualization with 3,3-diaminobenzidine (DAB; Dako). Before incubation with the Olig2 primary antibody, slides were blocked again with 5\% BSA for 60 minutes. Cells that stained positive for Olig2 were visualised by HistoGreen (Linaris, E109).

\section{Immunofluorescence}

To identify C. albicans in the brain tissue, immunofluorescence staining was performed as described previously using a rabbit anti-C. albicans antibody (Meridian Life Science, Memphis, TN, working concentration 1:50) and appropriate Alexa Fluor 594-labelled secondary antibody (working concentration 1:200). ${ }^{17}$ Briefly, sections were deparaffinized, rehydrated and incubated in $0.25 \%$ ammonia dissolved in $70 \%$ ethanol for one hour on a shaker. Antigen retrieval was performed using citrate buffer ( $\mathrm{pH}$ 6.0) in a microwave oven. Nonspecific binding was blocked by incubation with $10 \%$ goat serum for one hour followed by overnight incubation with primary anti-C.albicans antibody dissolved in $0.1 \%$ PBS with $0.2 \%$ Tween-20. For fluorescence staining, Alexa Fluor conjugated secondary antibody was used followed by incubation in $0.3 \%$ Sudan 
Black Solution to reduce auto-fluorescence. Counterstaining was done with the fluorescent nuclear marker Hoechst.

To assess neuronal injury, a Fluoro-Jade C staining was performed, which is a specific marker for identification of degenerating neurons. ${ }^{22,23}$ In this procedure, sections were deparaffinized, rehydrated and immersed in $0.06 \%$ potassium permanganate with $0.01 \%$ Hoechst for 10 minutes. Before immersing in the Fluoro-Jade $\mathrm{C}$ solution, sections were rinsed in running tap water for 1-2 minutes. Immersion in the Fluoro-Jade C solution (Millipore, AG325) ( $0.01 \%$ stock solution in $0.1 \%$ acetic acid) was performed in the dark for 10 minutes. Sections were washed with distilled water and subsequently air dried at $50^{\circ} \mathrm{C}$. Finally, sections were cleared with xylene for 1 minute before coverslipped using DPX.

\section{Qualitative analysis}

H\&E-stained sections were analysed by three independent investigators and two neuropathologists, blinded to the experimental groups, to assess overall brain structure and inflammatory changes. The absence of tissue autolysis in our sections was confirmed by neuropathologists. The sections were examined for the presence of hemorrhages, gliosis, (cytotoxic) oedema, abscess formation, and structural damage, including cyst formation. C. albicans fluorescent and PAS-stained sections were examined for the presence of $C$. albicans.

\section{Quantitative analysis}

For the analysis of IBA-1, GFAP, MBP, CNPase, Olig2, and CD68 immunoreactivity, digital images of the hippocampus, white matter (WM) and periventricular white matter (PVWM) were acquired using an Olympus AX-70 microscope (Olympus, Tokyo, Japan) equipped with a black and white digital camera. From each section, one picture of the hippocampus was taken at a 20x magnification and images in the WM were taken at a 100x magnification of the gliotic foci and 4-6 consecutive images of the PVWM. The area fraction of IBA-1, GFAP and MBP immuno-reactivity was determined with a standard intensity threshold to determine positive staining using Qwin Pro $\mathrm{V}$ 3.5.1 software (Leica, Rijswijk, the Netherlands). Blood vessels and artefacts were excluded from analysis. The CNPase and Olig2 positive cells were counted using Qwin software and expressed as cells $/ \mathrm{mm}^{2}$. Ki67, caspase-3, CD3 and MPO positive cells were counted in all brain regions, focussing on the cerebral vasculature. The digital images were acquired and analyzed by an independent observer who was blinded to the experimental groups. Fluoro-Jade $\mathrm{C} /$ Hoechst stainings were examined using the Olympus AX-70 fluorescent microscope. 


\section{Data analysis}

Statistical analysis was performed with GraphPad Prism software (version v5.0; GraphPad Software Inc., La Jolla, CA, USA). Results were analyzed using the nonparametric Kruskal-Wallis test with Dunnett's post-hoc testing to compare different groups. Results are given as mean and standard error of the mean (SEM) with significance at $p<0.05$.

\section{RESULTS}

\section{C. albicans culture}

C. albicans colonial morphology was confirmed by growth on Brilliance Candida Agar (Oxoid, Adelaide, Australia). Positive cerebrospinal fluid (CSF) cultures for C. albicans were detected in $67 \%(4 / 6)$ of the 3D C. albicans-exposed animals and in 50\% (3/6) of the 3D C. albicans/ Fluconazole-treated animals (Table 1). No positive cultures were present in the 5D C. albicans/Fluconazole-treated group (0/5). Control animals had negative CSF cultures. Results of amniotic fluid and blood cultures were previously published by Maneenil and colleagues and are also summarized in the table. ${ }^{16}$

Table 1 Candida albicans cultures.

\begin{tabular}{lllll}
\hline & Control & 3D C. albicans & $\begin{array}{l}\text { 3D C. albicans and } \\
\text { Fluconazole }\end{array}$ & $\begin{array}{l}\text { 5D C. albicans and } \\
\text { Fluconazole }\end{array}$ \\
\hline Cerebrospinal fluid & $0 / 6$ & $4 / 6$ & $3 / 6$ & $0 / 5$ \\
Fetal blood * & $0 / 6$ & $4 / 6$ & $5 / 6$ & $3 / 5$ \\
Amniotic fluid * & $0 / 6$ & $6 / 6$ & $6 / 6$ & $5 / 5$ \\
\hline
\end{tabular}

The ratio of C. albicans positive / negative cultures are depicted. ${ }^{*}$ Based on previously published data. ${ }^{16}$

No evidence of C. albicans invasion in the brain

Despite substantial numbers of animals with positive CSF cultures for C. albicans, PAS and immunofluorescent staining did not identify $C$. albicans in the parenchyma of the brains of any of the experimental groups (data not shown).

\section{Pro-inflammatory cytokine response}

Significantly elevated plasma IL-6 concentrations were found in 50\% (3/6) of the 3D C. albicans-exposed animals when compared to controls (Figure 2). 40\% (2/5) of the 5D C. albicans/Fluconazole-treated animals demonstrated elevated plasma concentrations of IL-6, however statistical significance was not reached. Plasma IL-6 concentrations in the 
controls, 1D Fluconazole- and 3D C. albicans/Fluconazole-treated animals were not detectable.

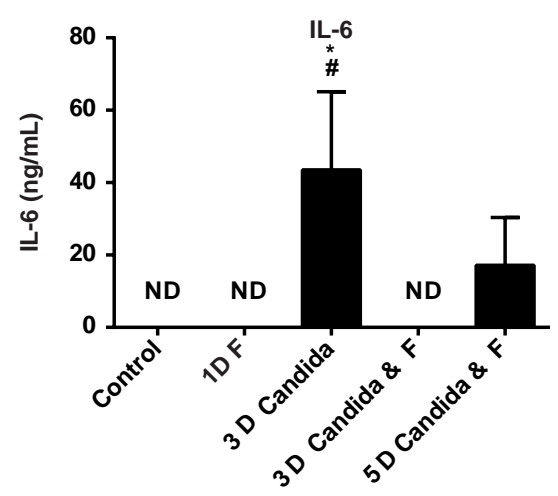

Figure 2. Plasma IL-6 concentrations following intra-amniotic C. albicans and/or Fluconazole treatment.

For statistical analysis, undetectable values were assigned an arbitrary value of $1 \mathrm{pg} / \mathrm{mL}$. ${ }^{*} \mathrm{P} \leq 0.05$ vs. control; \# P $\leq 0.05$ vs. 3D C. albicans/Fluconazole.

\section{Structural analysis of the brain}

Qualitative analysis of H\&E stained sections revealed no evidence of structural changes including intraventricular hemorrhages and cystic lesions in any of the experimental groups. However, the brains of $C$. albicans-exposed animals had increased cell density in the white matter which primarily consisted of glial cells and blood-derived macrophages, which cannot be distinguished from microglia (Figure 3). These gliotic lesions were primarily present in the gyral crests of the white matter. In addition, animals with gliosis showed marked vasodilatation and hyperemia (Figure 3D). These inflammatory alterations were more pronounced in the 5D C. albicans/Fluconazoletreated group (Figure $3 \mathrm{C}-\mathrm{D}$ ) compared to the 3D C. albicans-exposed (data not shown) and the control animals (Figure $3 \mathrm{~A}-\mathrm{B}$ ). Interestingly, no inflammatory alterations were detected in the subcortical white matter (Figure 3C). 

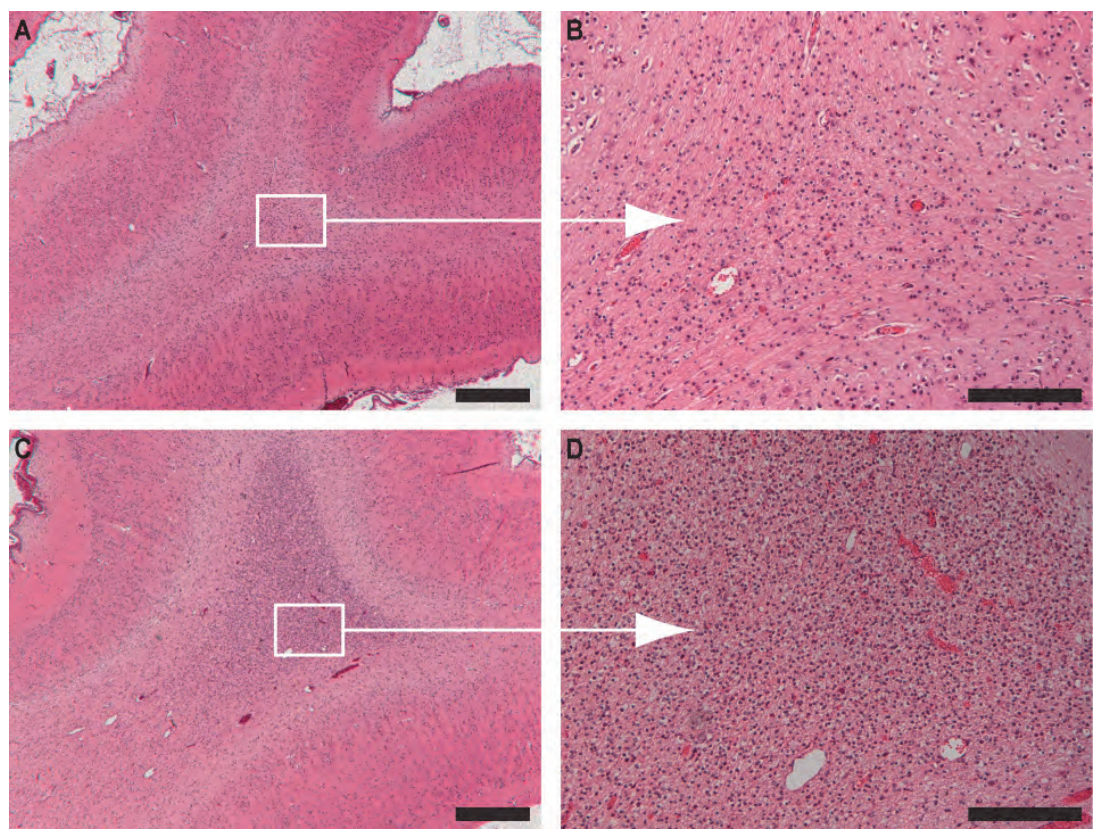

Figure 3 H\&E stained brain sections of control animals and 5D C. albicans/Fluconazole-treated animals.

Control animals $(A, B)$ showed no to mild gliosis and the 5D C. albicans/Fluconazole-treated animals (C,D) showed severe gliotic foci in white matter of the gyral crest. A and $C$ at $25 x$ magnification (scale bar $=500 \mu \mathrm{m}$ ) and $B$ and $D$ at 200x magnification (scale bar $=100 \mu \mathrm{m}$ ).

This finding is substantiated by an increase of IBA-1 immunoreactivity in the white matter after intra-amniotic exposure to C. albicans irrespective of Fluconazole treatment compared to control group and/or 1D Fluconazole group (Figure 4A-D). Immunoreactivity of CD68, representative of activated microglia ${ }^{24}$, was increased in the 5D C. albicans/Fluconazole group compared to controls (Figure $4 \mathrm{E}-\mathrm{H}$ ). No significant changes in IBA-1 immunoreactivity in the periventricular white matter were observed. However, in $17 \%(1 / 6)$ of the 3D C. albicans animals and $60 \%(3 / 5)$ of the $5 D C$. albicans/Fluconazole animals, foci containing CD68 positive cells were found in the periventricular white matter (data not shown). Besides an increase in IBA-1 immunoreactivity, an increase of the astrocyte marker glial fibrillary acidic protein (GFAP) was found in the hippocampus after intra-amniotic exposure to C. albicans compared to the control group and/or 1D Fluconazole group (Figure 5). Administration of Fluconazole irrespective of pre-exposure to C. albicans did not induce changes in IBA1 or GFAP immunoreactivity. 

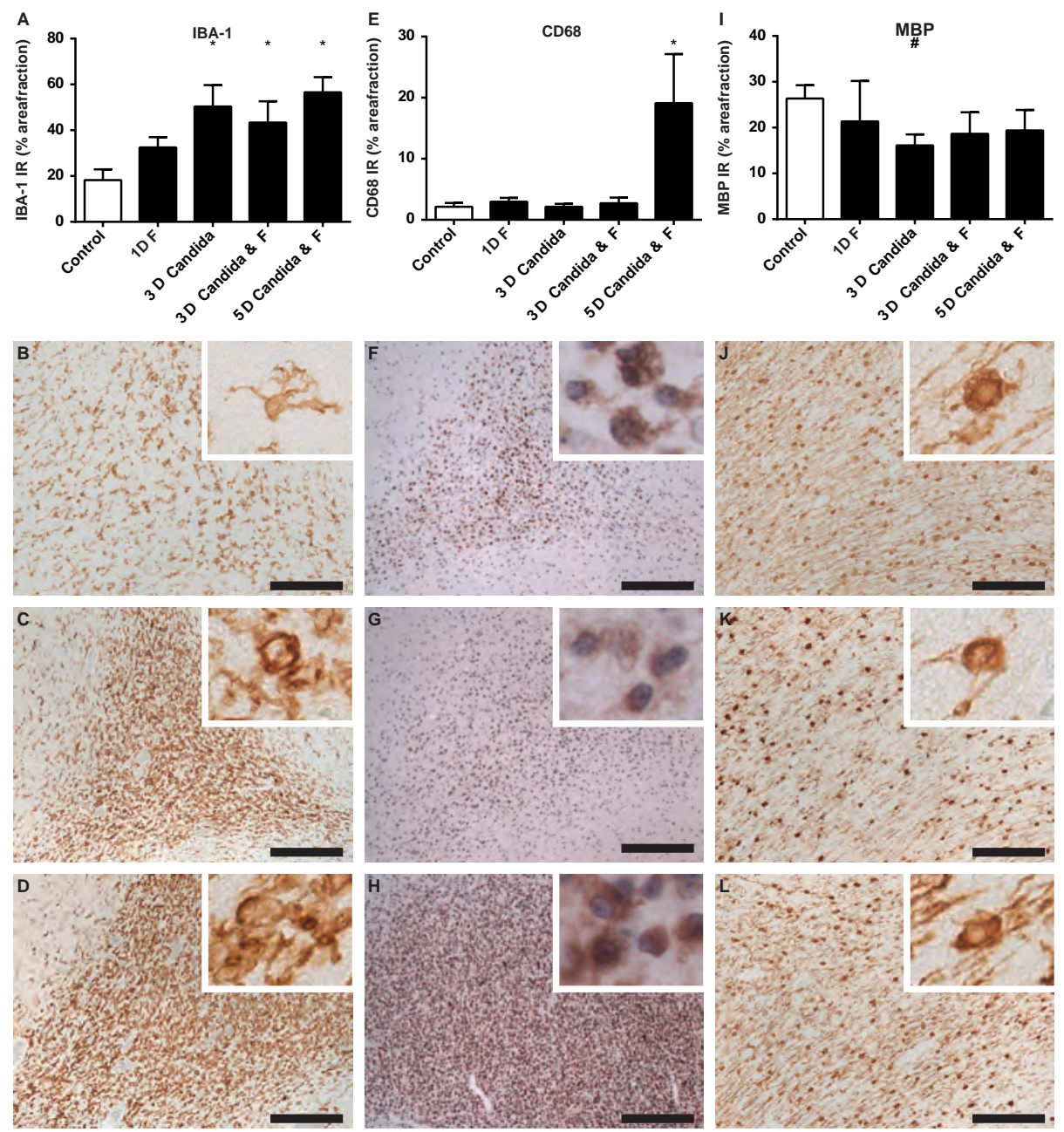

Figure 4 Antenatal exposure to $\mathrm{C}$. albicans caused microglia activation and subsequent myelin disturbances in the white matter.

Significant increase $(*, p<0.05)$ of the area fraction of IBA-1 (A-D) immunoreactivity (IR) was observed in all C. albicans-exposed animals compared to controls irrespective of Fluconazole (F) treatment. A decrease of MBP IR $(E-H)$ was observed which did not reached significance $(\#, p<0.1)$ in the 3D C. albicans-exposed group compared to the controls irrespective of Fluconazole treatment. Representative figures show that the area fraction of the IBA-1 IR is higher in the 3D C. albicans group (C) and 5D C. albicans/Fluconazole-treated group (D) compared to the control group (B). Morphological analysis showed a higher density of amoeboid microglia present in the white matter after $\mathrm{C}$. albicans exposure (inserts). In these regions with microglia activation, severe myelin disturbances and loss of myelin fibers were found in the 3D C. albicans-exposed (G) and the 5D C. albicans/Fluconazole-treated $(\mathrm{H})$ group. Figures of the 3D C. albicans/Fluconazole-treated group are not depicted here. Images taken at 100x magnification (insert at 400x magnification), scale bar $=500 \mu \mathrm{m}$. 
Chapter 4
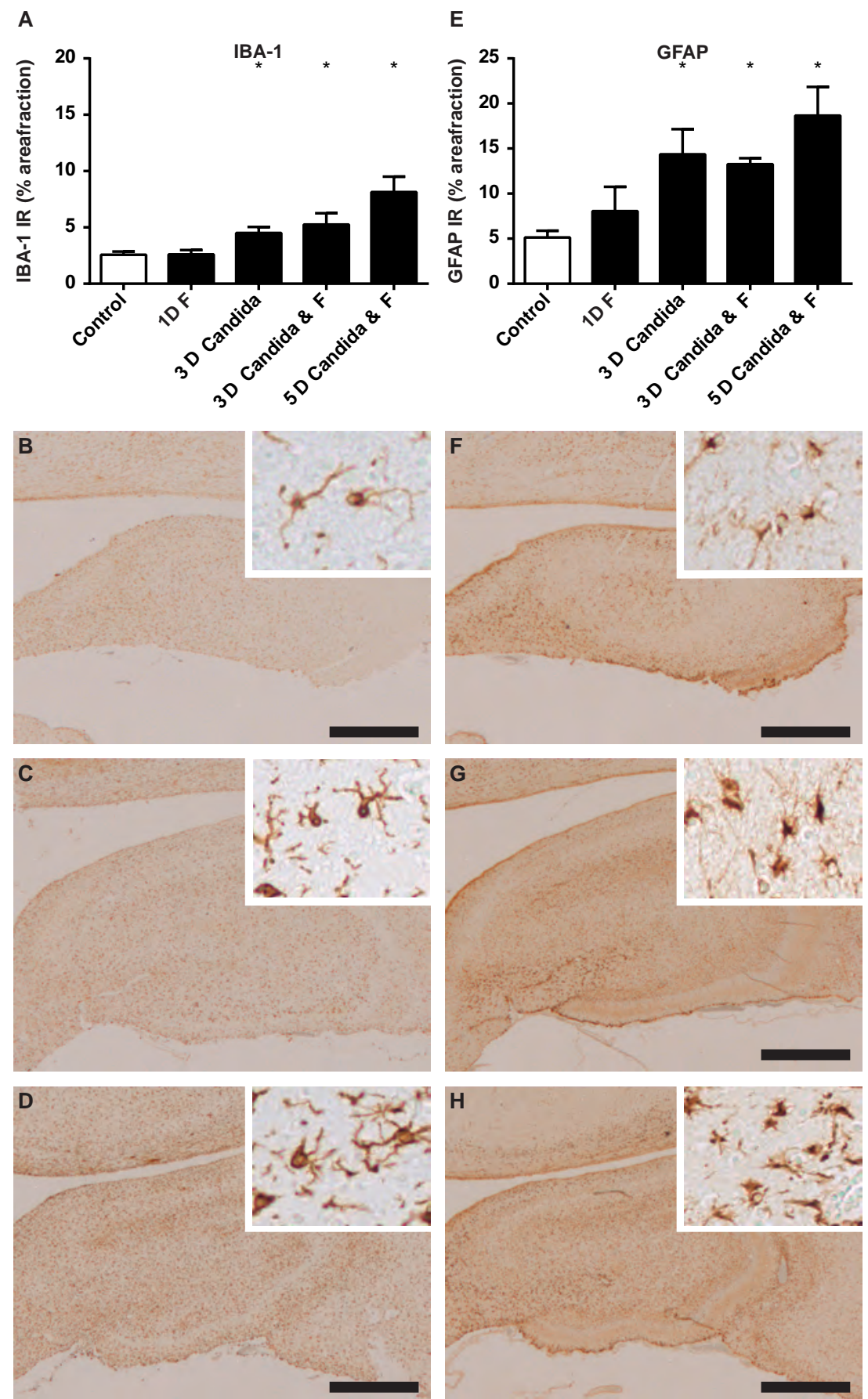

Figure 5 Antenatal exposure to Candida albicans caused microglial (IBA-1) and astrocyte (GFAP) activation in the hippocampus. 
A significant increase $(*, p<0.05)$ of the area fraction of IBA-1 (A-D) and GFAP $(E-H)$ immunoreactivity $(I R)$ was observed in all C. albicans-exposed animals compared to controls irrespective of Fluconazole (F) treatment $(A, E)$. No significant changes were found in the Fluconazole treated animals compared to controls. Representative figures show that the area fraction of the IBA- 1 IR and GFAP IR was higher in the 3D C. albicans group $(C, G)$ and $5 D C$. albicans/Fluconazole-treated group $(D, H)$ compared to the control group $(B, F)$. Images at 20x magnification (inserts at 200x magnification), scale bar $=100 \mu \mathrm{m}$.

\section{Antenatal exposure to C. albicans caused focal white matter disturbances with loss of mature oligodendrocytes.}

There was reduced MBP immunoreactivity in regions with overt microglial activation. In particular, myelin was significantly disturbed in 5D C. albicans-exposed animals; in comparison to no myelin disturbances in control animals (Figure $4 \mathrm{I}-\mathrm{L}$ ). The mature oligodendrocytes had severely disturbed and loss of myelin fibres in these areas of injury. The apparent loss of MBP IR prompted us to further investigate the ratio between mature and total oligodendroglial lineage cells. Double staining for CNPase and Olig2 revealed a decrease of CNPase positive cells whereas the number of Olig2 positive cells remained identical, thereby resulting in a decreased ratio of mature versus total oligodendrocytes (Figure $6 \mathrm{~A}-\mathrm{C}$ ).

\section{Antenatal exposure to C. albicans did not result in neuronal degeneration}

In all experimental groups no Fluoro-Jade C positive neurons were detected throughout the brain sections (data not shown).

\section{$M P O$ and $C D 3$}

MPO- and CD3-positive cells were sporadically observed in the cerebral vasculature of the choroid plexus and meninges. No MPO- and CD3-positive cells were found in the cerebral white matter. No differences were seen between all experimental groups (supplementary figure 1). 

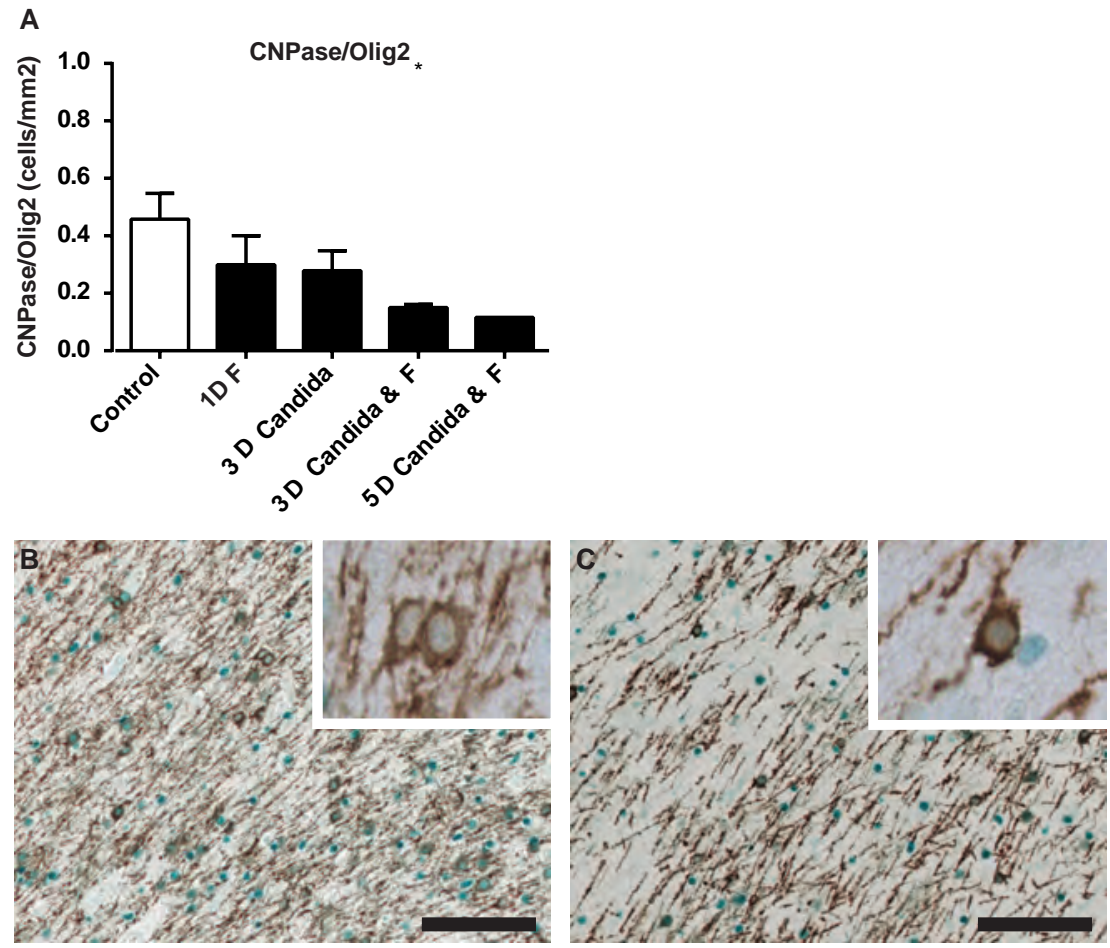

Figure 6 Myelin disturbances are accompanied by a loss of mature oligodendrocytes.

A significant decrease $(*, p<0.05)$ of the ratio between CNPase positive mature oligodendrocytes and total number of cells of oligodendroglial lineage (Olig2 positive) was observed following 3D C. Albicans with Fluconazole (F) treatment and 5D C. albicans-exposed groups with Fluconazole treatment compared to controls. (A) Graphical representation of the CNPase/Olig2 ration; (B-C) representative figures of CNPase/Olig2 double stain in (B) controls and (C) 5D C. Albicans-exposed animals indicating an apparent loss of CNPase positive cells. Images taken at 200x magnification (insert at 400x magnification), scale bar $=100 \mu \mathrm{m}$.

\section{Ki67 and caspase 3}

An increase in Ki67 and caspase-3 positive cells was found in the white matter and hippocampus of the 5D C. albicans/Fluconazole group compared to controls (Figure $7 \mathrm{~A}$ $\mathrm{H}$, Table 2). Moreover, in the periventricular white matter, an increase in caspase-3 positive cells was observed. 


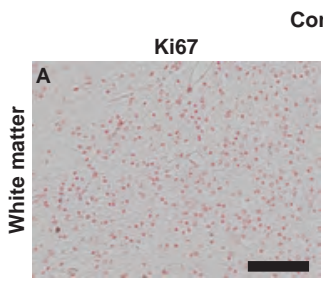

Control
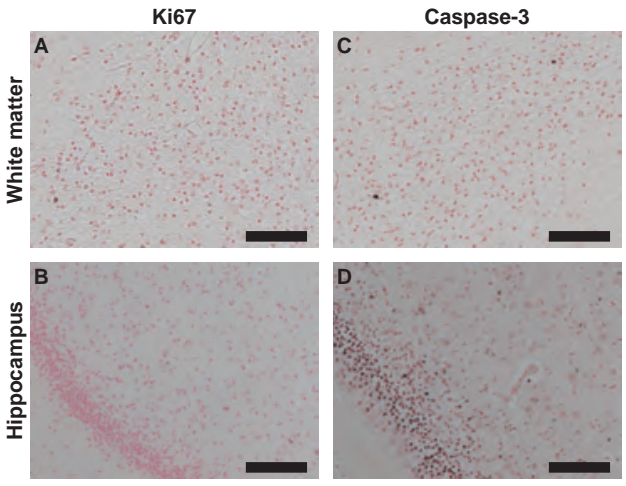

5D Candida \& $\mathrm{F}$

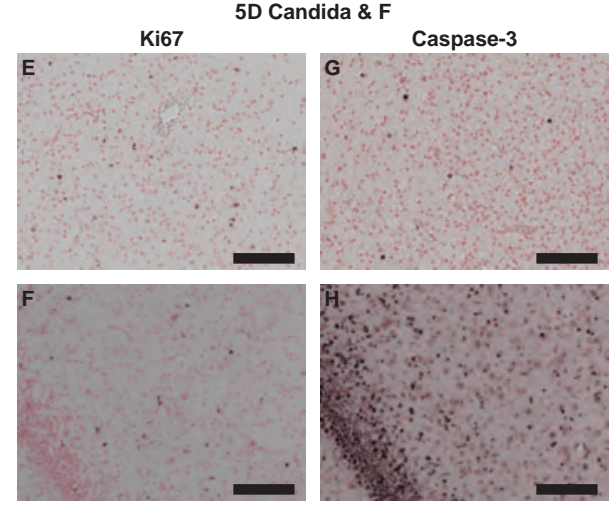

Figure 7 Antenatal exposure to Candida albicans resulted in increased proliferation and apoptosis in the subcortical white matter and hippocampus.

A significant increase $(*, p<0.05)$ of KI67 and caspase-3 positive cells were observed following 5D C. Albicans with Fluconazole treatment in the white matter $(E, G)$ and hippocampus $(F, H)$ compared to controls $(A, B, C, D)$. Images taken at 200x magnification, scale bar $=100 \mu \mathrm{m}$.

Table 2 Ki67 and Caspase-3 postive cells.

\begin{tabular}{|c|c|c|c|c|c|}
\hline & Control & 1D Fluconazole & 3D C. albicans & $\begin{array}{l}\text { 3D C. albicans } \\
\text { and Fluconazole }\end{array}$ & $\begin{array}{l}\text { 5D C. albicans } \\
\text { and Fluconazole }\end{array}$ \\
\hline \multicolumn{6}{|l|}{ Ki67 + cells $/ \mathrm{mm}^{2}$} \\
\hline White matter & $\begin{array}{l}11.16 \\
(7.21)\end{array}$ & $\begin{array}{l}41.58 \\
(38.68)\end{array}$ & $\begin{array}{l}21.85 \\
(16.40)\end{array}$ & $\begin{array}{l}54.69 \\
(70.95)\end{array}$ & $\begin{array}{l}55.99 \\
(31.10) *\end{array}$ \\
\hline $\begin{array}{l}\text { Periventricular white } \\
\text { matter }\end{array}$ & $\begin{array}{l}7.585 \\
(9.14)\end{array}$ & $\begin{array}{l}14.41 \\
(11.22)\end{array}$ & $\begin{array}{l}17.61 \\
(14.20)\end{array}$ & $\begin{array}{l}8.597 \\
(10.74)\end{array}$ & $\begin{array}{l}20.90 \\
(18.87)\end{array}$ \\
\hline Hippocampus & $\begin{array}{l}5.58 \\
(4.16)\end{array}$ & $\begin{array}{l}16.75 \\
(13.61)\end{array}$ & $\begin{array}{l}22.15 \\
(27.54)\end{array}$ & $\begin{array}{l}13.07 \\
(12.98)\end{array}$ & $\begin{array}{l}33.43 \\
(27.00) *\end{array}$ \\
\hline \multicolumn{6}{|l|}{ Caspase $3+$ cells $/ \mathrm{mm}^{2}$} \\
\hline White matter & $\begin{array}{l}12.80 \\
(13.60)\end{array}$ & $\begin{array}{l}14.08 \\
(11.12)\end{array}$ & $\begin{array}{l}23.68 \\
(33.21)\end{array}$ & $\begin{array}{l}16.87 \\
(6.76)\end{array}$ & $\begin{array}{l}40.22 \\
(22.58) *\end{array}$ \\
\hline $\begin{array}{l}\text { Periventricular white } \\
\text { matter }\end{array}$ & $\begin{array}{l}26.14 \\
(11.40)\end{array}$ & $\begin{array}{l}35.42 \\
(24.41)\end{array}$ & $\begin{array}{l}93.44 \\
(110.20)\end{array}$ & $\begin{array}{l}46.44 \\
(29.12)\end{array}$ & $\begin{array}{l}155.10 \\
(22.36)^{*}\end{array}$ \\
\hline Hippocampus & $\begin{array}{l}364.3 \\
(175.30)\end{array}$ & $\begin{array}{l}264.4 \\
(19.56)\end{array}$ & $\begin{array}{l}443.2 \\
(198.80)\end{array}$ & $\begin{array}{l}313.4 \\
(117.00)\end{array}$ & $\begin{array}{l}726.30 \\
(317.90)^{*}\end{array}$ \\
\hline
\end{tabular}

Ki67 and Caspase-3 positive cells expressed as mean $\left( \pm\right.$ SD). ${ }^{*}<0.05$ versus control

\section{DISCUSSION}

The main finding of this study is that 3 days of intra-amniotic exposure to C. albicans resulted in increased microglial and astrocyte activation with subsequent focal white matter disturbances in the preterm ovine brain. At this stage, C. albicans positive CSF cultures were detected without $C$. albicans-positive immunostainings of the brain. This 
may indicate that cerebral inflammation and injury in the acute phase after intraamniotic C. albicans delivery is not caused by invasion of $C$. albicans in the brain parenchyma. ${ }^{25,26}$ This concept is supported by the absence of neutrophils in the brain parenchyma since neutrophils are known to aggravate disruption of the blood-brain barrier promoting further invasion of $C$. albicans in the brain. ${ }^{25}$ However, we cannot rule out the possibility that low titers invaded the brain, since the initial phase of $C$. albicans invasion does not require a disruption of the blood-brain barrier and such low fungal burden of the brain cannot be detected by histology or immunofluorescence. ${ }^{15,25}$

Since C. albicans was not detected in the fetal brain, we considered that severe systemic immune activation, as shown in the present and previous studies ${ }^{16,17}$, was (primarily) responsible for the acute changes observed in the fetal brain 3 days post $C$. albicans infection of the amniotic cavity for the following reasons: Pro-inflammatory cytokines, such as IL-6, contribute to activation of the endothelium of the blood-brain barrier, which in turn activate adjacent microglia and astrocytes, ultimately resulting in a cerebral inflammatory response. ${ }^{27-31}$ In line with this, systemic inflammation is known to be causally involved in the induction of white matter injury - as seen in our model. ${ }^{32}$, ${ }^{33}$ In addition, IL-6 was shown to be involved in the inhibition of neurogenesis and the development of hyper-excitable neurological conditions including epilepsy, psychoses, anxiety and autism spectrum disorders in experimental models. ${ }^{28,34}$

Notably, increased circulatory IL-6 levels were not always paralleled by positive blood cultures in our study. This finding can be explained by earlier studies in which the presence of a pro-inflammatory trigger in the amniotic fluid caused systemic inflammation without migration of microorganisms or their components into the fetal circulation. $^{35,36}$

Intra-amniotic C. albicans caused a heterogeneous pattern of cerebral immune activation and microgliosis. Moreover, microglial activation, as identified by immunohistochemical analysis of CD68, was strongly associated with white matter injury within these specific regions. An identical pattern of systemic immune activation, neuroinflammation and concomitant structural injury was found following intraamniotic delivery of endotoxin in the same animal model. ${ }^{21,37-39}$ This indicates that irrespective of the inflammatory trigger responsible for the onset of the fetal inflammatory response, inflammation as such, and not the stimulus of inflammation, appears to be the most important factor in inflammation-induced fetal brain injury within the first days after induction of chorioamnionitis.

White matter injury can occur as a result of activation of the cerebral immune system, characterized by activated microglia and astrocytes, which produce cytokines, free radicals, nitric oxide, and excitotoxic amino acids, all of which are detrimental to the survival of oligodendrocytes. ${ }^{7}, 40$ The reduced CNPase/Olig2 ratio indicates that white matter injury in our model was most likely the result of death of mature oligodendrocytes. This loss was 
paralleled by increased proliferation of oligodendrocyte progenitors, potentially as compensation for the observed reduction of mature oligodendrocytes. ${ }^{41,42}$ It is tempting to speculate that these progenitors might fail to mature into functional myelin producing oligodendrocyte since this phenotype has previously been reported in experimental models of perinatal stress ${ }^{43-45}$ and in human neonates with periventricular leukomalacia. ${ }^{41}$ Finally, such oligodendrocyte maturation arrest correlates with motor deficits characteristic for cerebral palsy. ${ }^{40,45,46}$ In conjunction with white matter injury, grey matter injury plays an independent role in neurodevelopmental outcome. ${ }^{35}$ However, in our study no degenerative neurons were observed. ${ }^{22,23}$ In addition, cerebral palsy also comprises cognitive impairments ${ }^{7,8}$ for which we analyzed the hippocampus in which a similar inflammatory response was observed which is consistent with our previous results. ${ }^{21,39}$

We previously reported that intra-amniotic C. albicans rapidly colonizes the amniotic fluid, causing inflammation of the fetal skin and lungs, and progresses into a fetal systemic inflammatory response causing fetal death within 5 days after exposure. ${ }^{16,17}$ In the present study we show that Fluconazole administration eradicated C. albicans from the CSF and reduced systemic inflammation. Importantly, reduction of the fungal burden and inhibition of systemic immune activation following Fluconazole treatment was not accompanied by attenuation of cerebral inflammation and injury. Multiple studies have demonstrated that cerebral inflammation acquired during early fetal development continues postnatally and might even persist into adulthood. ${ }^{47,} 48$ Therefore, we consider that the observed cerebral inflammatory response following intra-amniotic $C$. albicans exposure is a persistent reaction that is initiated by peripheral immune activation. Although Fluconazole treatment did not protect against structural cerebral injury in this acute phase, the observed inhibition of systemic immune activation might be clinically relevant, since prolonged and aberrant systemic immune activation are known to induce blood-brain barrier disruption, thereby facilitating cerebral invasion of $C$. albicans and substantially aggravating cerebral injury resulting in increased morbidity and mortality. ${ }^{26}$ Collectively, previous and current findings suggest that timing of infection and the start of antifungal treatment regimen are important in the initiation, maintenance, and possible resolution of neuroinflammation and structural injury in the fetal brain. The current study provides important insight in the sequelae of events that contribute to induction of adverse outcomes of the premature brain following intra-amniotic C. albicans infection. In addition, this study points out that Fluconazole treatment should be started immediately after intra-amniotic exposure to $C$. albicans considering the narrow therapeutic time window to reduce morbidity and mortality. Therefore, further research should focus on timing, frequency and dosing of antifungals in the presence or absence of immunomodulatory treatment. $^{49}$ 
Fluconazole treatment was not associated with adverse effects in our study, which is in line with the findings of Maneenil and colleagues in the same model ${ }^{16}$ and studies demonstrating that teratogenic effects of Fluconazole are exclusively seen when used in the first trimester at higher doses and after multiple gifts. ${ }^{50}$ The administered Fluconazole dose in this study mimics the amount used in systemic neonatal candidiasis in which no adverse long-term brain pathology and behavioral deficits are currently described. ${ }^{50}$ In addition, clinical in utero administration of Fluconazole prolonged pregnancy in two cases of intra-amniotic C. albicans infection without adverse treatment effects on the fetus. ${ }^{13}$

We note that our study has several limitations. We only tested one dose and frequency of Fluconazole administration. In addition, we were not able to correlate histological changes to functional neurological outcomes. However, several studies previously established an association between endotoxin-induced chorioamnionitis and functional EEG changes. ${ }^{44,51,52}$

\section{Conclusion}

We have shown that intra-amniotic exposure to $C$. albicans results in an acute systemic and neuroinflammatory response with concomitant white matter injury. Although systemic immune activation was significantly inhibited following Fluconazole treatment, modulation of the cerebral inflammatory response and prevention of concomitant white matter injury was not found.

This study forms the essential basis for follow up studies in which the timing, frequency and dosing of antifungal treatment must be explored and alternative/additional treatment options including immunomodulatory interventions can be tested. 


\section{SUPPLEMENTARY FIGURES}
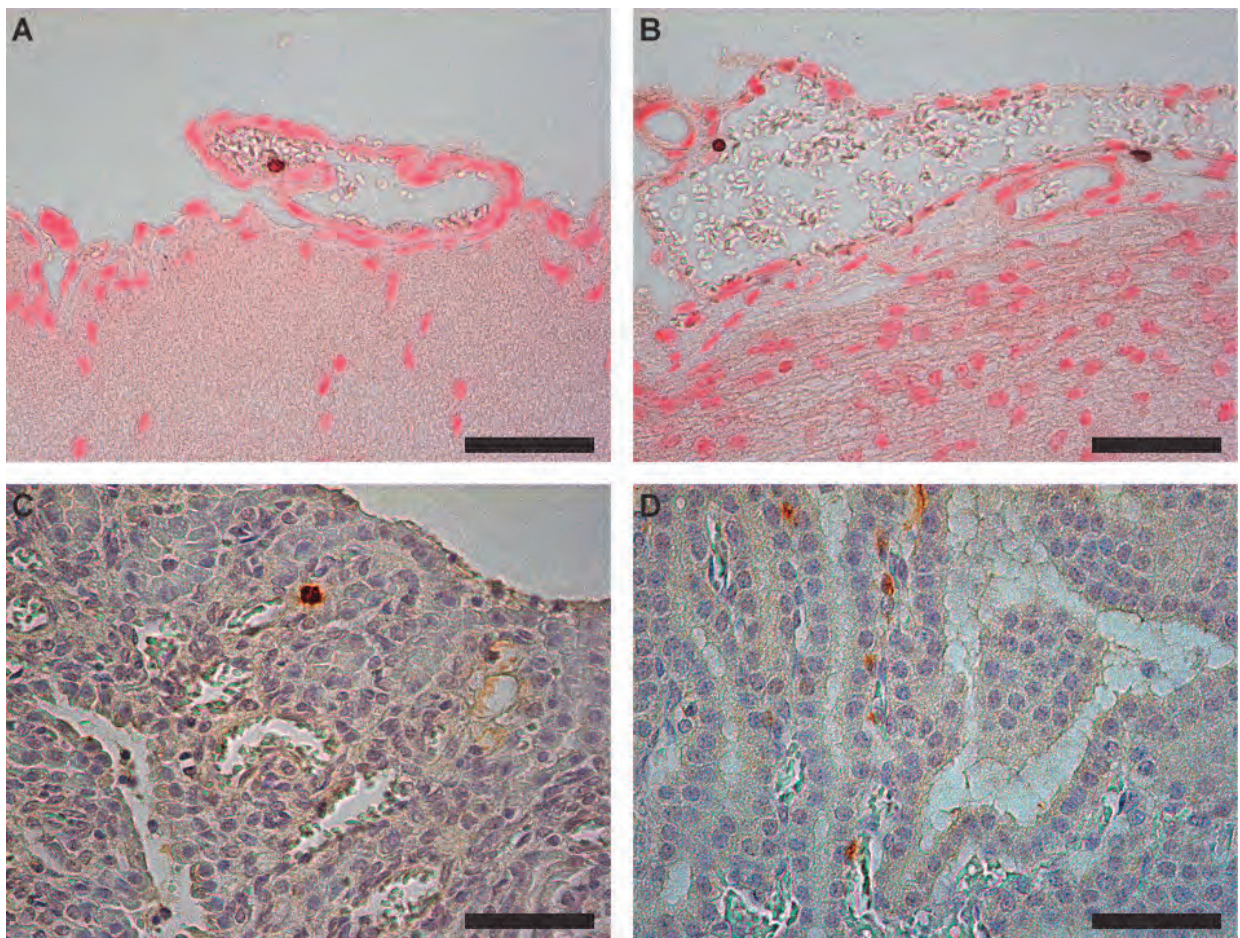

Supplementary figure 1 Antenatal exposure to $\mathrm{C}$. albicans does not result in infiltration of peripheral immune cells.

(A-B) representative pictures of CD3 positive T lymphocytes in the meninges of (A) controls and (B) 5D C. Albicans /Fluconazole animals. (C-D) representative pictures of MPO positive neutrophils in the choroid plexus of (C) controls and (D) 5D C. Albicans /Fluconazole animals. 


\section{REFERENCES}

1. Agrawal, V. \& Hirsch, E. Intrauterine infection and preterm labor. Seminars in fetal \& neonatal medicine 17, 12-19 (2012).

2. Goldenberg, R.L., Culhane, J.F., lams, J.D. \& Romero, R. Epidemiology and causes of preterm birth. Lancet 371, $75-84$ (2008).

3. Goldenberg, R.L., Hauth, J.C. \& Andrews, W.W. Intrauterine infection and preterm delivery. N Engl J Med 342, 1500-1507 (2000).

4. Galinsky, R., Polglase, G.R., Hooper, S.B., Black, M.J. \& Moss, T.J. The consequences of chorioamnionitis: preterm birth and effects on development. Journal of pregnancy 2013, 412831 (2013).

5. Gantert, M. et al. Chorioamnionitis: a multiorgan disease of the fetus? J Perinatol 30 Suppl, S21-30 (2010).

6. Malaeb, S. \& Dammann, O. Fetal inflammatory response and brain injury in the preterm newborn. Journal of child neurology 24, 1119-1126 (2009).

7. Kuypers, E. et al. White matter injury following fetal inflammatory response syndrome induced by chorioamnionitis and fetal sepsis: lessons from experimental ovine models. Early human development 88, 931-936 (2012).

8. Wu, Y.W. Systematic review of chorioamnionitis and cerebral palsy. Mental retardation and developmental disabilities research reviews 8, 25-29 (2002).

9. Chau, V., McFadden, D.E., Poskitt, K.J. \& Miller, S.P. Chorioamnionitis in the pathogenesis of brain injury in preterm infants. Clinics in perinatology 41, 83-103 (2014).

10. Yoon, B.H. et al. Fetal exposure to an intra-amniotic inflammation and the development of cerebral palsy at the age of three years. American journal of obstetrics and gynecology 182, 675-681 (2000).

11. DiGiulio, D.B. et al. Microbial prevalence, diversity and abundance in amniotic fluid during preterm labor: a molecular and culture-based investigation. PLoS One 3, e3056 (2008).

12. Payne, M.S. \& Bayatibojakhi, S. Exploring preterm birth as a polymicrobial disease: an overview of the uterine microbiome. Frontiers in immunology 5 (2014).

13. Bean, L.M., Jackson, J.R., Dobak, W.J., Beiswenger, T.R. \& Thorp, J.A. Intra-amniotic fluconazole therapy for Candida albicans intra-amniotic infection. Obstetrics and gynecology 121, 452-454 (2013).

14. Meizoso, T. et al. Intrauterine candidiasis: report of four cases. Archives of gynecology and obstetrics 278, 173-176 (2008).

15. Tsai, N.Y., Laforce-Nesbitt, S.S., Tucker, R. \& Bliss, J.M. A murine model for disseminated candidiasis in neonates. Pediatric research 69, 189-193 (2011).

16. Maneenil, G. et al. Fluconazole Treatment of Intrauterine Candida albicans Infection in Fetal Sheep. Pediatric research (2015).

17. Payne, M.S. et al. Intrauterine Candida albicans infection elicits severe inflammation in fetal sheep. Pediatric research 75, 716-722 (2014).

18. Moudgal, V.V. \& Sobel, J.D. Antifungal drugs in pregnancy: a review. Expert opinion on drug safety 2, 475483 (2003).

19. Collins, J.J. et al. Repeated intrauterine exposures to inflammatory stimuli attenuated transforming growth factor-beta signaling in the ovine fetal lung. Neonatology 104, 49-55 (2013).

20. Back, S.A., Riddle, A. \& Hohimer, A.R. Role of instrumented fetal sheep preparations in defining the pathogenesis of human periventricular white-matter injury. Journal of child neurology 21, 582-589 (2006).

21. Gavilanes, A.W. et al. Chorioamnionitis induced by intraamniotic lipopolysaccharide resulted in an interval-dependent increase in central nervous system injury in the fetal sheep. American journal of obstetrics and gynecology 200, 437 e431-438 (2009).

22. Rocha, E., Hammond, R. \& Richardson, B. Necrotic cell injury in the preterm and near-term ovine fetal brain after intermittent umbilical cord occlusion. American journal of obstetrics and gynecology 191, 488-496 (2004). 
23. Schmued, L.C., Stowers, C.C., Scallet, A.C. \& Xu, L. Fluoro-Jade C results in ultra high resolution and contrast labeling of degenerating neurons. Brain research 1035, 24-31 (2005).

24. Perego, C., Fumagalli, S. \& De Simoni, M.-G. Temporal pattern of expression and colocalization of microglia/macrophage phenotype markers following brain ischemic injury in mice. Journal of neuroinflammation 8, 174 (2011).

25. Navarathna, D.H. et al. MRI confirms loss of blood-brain barrier integrity in a mouse model of disseminated candidiasis. NMR in biomedicine 26, 1125-1134 (2013).

26. Lionakis, M.S., Lim, J.K., Lee, C.C. \& Murphy, P.M. Organ-specific innate immune responses in a mouse model of invasive candidiasis. Journal of innate immunity 3, 180-199 (2011).

27. Rivest, S. Molecular insights on the cerebral innate immune system. Brain, behavior, and immunity 17 , 13-19 (2003)

28. Deverman, B.E. \& Patterson, P.H. Cytokines and CNS development. Neuron 64, 61-78 (2009).

29. Dammann, O. \& O'Shea, T.M. Cytokines and perinatal brain damage. Clinics in perinatology 35, 643-663, $\mathrm{v}(2008)$.

30. Hoogland, I.C., Houbolt, C., van Westerloo, D.J., van Gool, W.A. \& van de Beek, D. Systemic inflammation and microglial activation: systematic review of animal experiments. J Neuroinflammation 12, 114 (2015).

31. Biesmans, S. et al. Systemic immune activation leads to neuroinflammation and sickness behavior in mice. Mediators of inflammation 2013, 271359 (2013).

32. Gomez, R. et al. The fetal inflammatory response syndrome. American journal of obstetrics and gynecology 179, 194-202 (1998).

33. Rees, S. \& Inder, T. Fetal and neonatal origins of altered brain development. Early human development 81, 753-761 (2005).

34. Atzori, M., Garcia-Oscos, F. \& Mendez, J.A. Role of IL-6 in the etiology of hyperexcitable neuropsychiatric conditions: experimental evidence and therapeutic implications. Future medicinal chemistry 4, 2177 2192 (2012).

35. Wolfs, T.G. et al. Chorioamnionitis-induced fetal gut injury is mediated by direct gut exposure of inflammatory mediators or by lung inflammation. American journal of physiology. Gastrointestinal and liver physiology 306, G382-393 (2014).

36. Kemp, M.W. et al. Selective exposure of the fetal lung and skin/amnion (but not gastro-intestinal tract) to LPS elicits acute systemic inflammation in fetal sheep. PloS one 8, e63355 (2013).

37. Nitsos, I. et al. Chronic exposure to intra-amniotic lipopolysaccharide affects the ovine fetal brain. Journal of the Society for Gynecologic Investigation 13, 239-247 (2006).

38. Rees, S. et al. Erythropoietin is neuroprotective in a preterm ovine model of endotoxin-induced brain injury. Journal of neuropathology and experimental neurology 69, 306-319 (2010).

39. Kuypers, E. et al. Effects of intra-amniotic lipopolysaccharide and maternal betamethasone on brain inflammation in fetal sheep. Plos one 8, e81644 (2013).

40. Volpe, J.J. Brain injury in premature infants: a complex amalgam of destructive and developmental disturbances. The Lancet. Neurology 8, 110-124 (2009).

41. Billiards, S.S. et al. Myelin abnormalities without oligodendrocyte loss in periventricular leukomalacia. Brain pathology 18, 153-163 (2008).

42. Filipovic, R. \& Zecevic, N. Lipopolysaccharide affects Golli expression and promotes proliferation of oligodendrocyte progenitors. Glia 49, 457-466 (2005).

43. Back, S.A. \& Miller, S.P. Brain injury in premature neonates: A primary cerebral dysmaturation disorder? Annals of neurology 75, 469-486 (2014).

44. Dean, J.M. et al. Delayed cortical impairment following lipopolysaccharide exposure in preterm fetal sheep. Annals of neurology 70, 846-856 (2011).

45. Segovia, K.N. et al. Arrested oligodendrocyte lineage maturation in chronic perinatal white matter injury. Annals of neurology 63, 520-530 (2008).

46. Buser, J.R. et al. Arrested preoligodendrocyte maturation contributes to myelination failure in premature infants. Annals of neurology 71, 93-109 (2012). 


\section{Chapter 4}

47. Fleiss, B. \& Gressens, P. Tertiary mechanisms of brain damage: a new hope for treatment of cerebral palsy? The Lancet. Neurology 11, 556-566 (2012).

48. Dammann, O. Persistent neuro-inflammation in cerebral palsy: a therapeutic window of opportunity? Acta paediatrica 96, 6-7 (2007).

49. Wolfberg, A.J., Dammann, O. \& Gressens, P. Anti-inflammatory and immunomodulatory strategies to protect the perinatal brain. Seminars in fetal \& neonatal medicine 12, 296-302 (2007).

50. Alsaad, A.M., Kaplan, Y.C. \& Koren, G. Exposure to fluconazole and risk of congenital malformations in the offspring: A systematic review and meta-analysis. Reproductive toxicology 52, 78-82 (2015).

51. Keogh, M.J. et al. Subclinical exposure to low-dose endotoxin impairs EEG maturation in preterm fetal sheep. American journal of physiology. Regulatory, integrative and comparative physiology 303, R270278 (2012).

52. Gavilanes, A.W. et al. Increased EEG delta frequency corresponds to chorioamnionitis-related brain injury. Frontiers in bioscience 2, 432-438 (2010). 


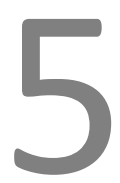

General Discussion 

Injury of the developing brain has been strongly correlated with morbidity and mortality in premature and full-term infants, resulting in an enormous physical, psychological and economic burden. ${ }^{1,2}$

Both intra-uterine infection and hypoxia-ischemia $(\mathrm{HI})$ are major causes of injury of the preterm brain. Although different in etiology, these conditions initiate a detrimental inflammatory response in the brain, leading to structural injury and subsequent dysfunction of the fetal brain.

Currently, treatment strategies are limited and mostly focused on $\mathrm{HI}$ in term infants, thereby excluding a large group of patients, indicating that novel therapeutic strategies are urgently warranted.

In this thesis, we assessed novel therapeutic strategies for injury of the preterm brain caused by either global hypoxia-ischemia or intra-uterine infection. Therefore, we postulated that therapies for hypoxia-ischemia-induced injury of the preterm brain should have both immune-modulatory and regenerative potential. These requirements are met by stromal cells. Furthermore, we assessed antimicrobial treatment as treatment strategy for intra-uterine infection-induced injury of the preterm brain.

We chose to test our therapeutic interventions in ovine models of global $\mathrm{HI}$ or intrauterine infection since ovine developmental biology closely resembles human developmental biology and adequately mimics the etiology of brain injury. ${ }^{3-5}$ Moreover, the long gestational period ( 147 days) enables us to perform detailed analyses of developmental processes at specific points during gestation. Furthermore, the size of the fetus allows instrumentation, continuous in utero registration of vital parameters with strong relevance and predictive values ${ }^{5-7}$ (i.e. blood pressure, electroencephalography, and electrocardiography), and application of intra-uterine treatment strategies.

\section{CELL-BASED THERAPIES}

Cell-based interventions have shown great promise as a therapeutic strategy for injury to the (neonatal) central nervous system. ${ }^{8-16}$ In previous studies we have shown that global $\mathrm{HI}$ injury of the preterm brain is mediated by a two-stepped inflammatory response in which the first inflammatory hit comprises a microglia-mediated local neuroinflammatory response followed by a systemic immune response, mediated by spleen-derived immune cells. ${ }^{3}$ Systemic administration of mesenchymal stromal cells (MSCs), before initiation of systemic immune activation, protected the preterm brain through reduction of microglial proliferation, induction of $\mathrm{T}$ cell tolerance, and reduction of cerebral invasion by peripheral immune-effector cells. ${ }^{17}$ 
However, clinical administration of cell-based therapies during early reperfusion mandates availability of large numbers of cells that can readily be administered, excluding the possibility of autologous transplantation.

Multipotent Adult Progenitor Cells (MAPCs) are adherent bone marrow-derived cells of an earlier developmental stage than MSC that have a high expansion potential with stable genetics. ${ }^{18,19}$ Furthermore, their immunological properties make it possible to use them as a universal allogeneic donor. ${ }^{18,20,21}$ Moreover, compared to MSCs, MAPC cells have stronger anti-inflammatory and regenerative potential. ${ }^{22-27}$

In chapter 2 we tested the neuroprotective potential of systemic administration of MAPC cells following global HI. In line with our earlier findings, we found that systemic administration of MAPC cells protected against structural and functional injury of the preterm brain. Interestingly, functional protection reached as far as the brainstem (i.e. baroreceptor reflex). Conservation of these functions indicates that structural protection by MAPC treatment also comprises protection of (deep) grey matter structures.

Also, in line with previous results, splenic involution, which is postulated to be caused by efflux of immune effector cells, was prevented by systemic MAPC administration. MAPC cells exert a plethora of neuroprotective functions that each engage at different time points in the pathophysiology of hypoxia-ischemia-induced injury of the preterm brain. Thus, timing of MAPC delivery is important to achieve specific treatment effects. We administered MAPC cells 1 hour and 4 days after the global HI insult. Inflammatory process including immune cell activation and recruitment are initiated almost immediately following global hypoxia-ischemia. Therefore, the first dose of MAPC cells was aimed to target immune-mediated secondary injury that is initiated during early reperfusion. A pilot study in this model demonstrated a peak in seizure burden in untreated animals at 4 days post hypoxia-ischemia. Therefore, we administered a second dose a 4 days post hypoxia-ischemia. Furthermore, a previous study demonstrated beneficial effects of systemically administered MAPC cells in a dosedependent manner. ${ }^{25,27}$

In the current study we assessed outcomes 7 days after hypoxia-ischemia, with favorable effects of MAPC treatment. Long-term evaluation, including postnatal administration, may further enhance the clinical significance of these results. A recent clinical trial in which stroke patients received systemic treatment with MultiStem ${ }^{\circledR}$, the clinical-grade product of MAPC cells, showed no difference in outcomes compared to placebo at 90 days. ${ }^{28}$ However, the analysis at 365 days showed a substantial favorable effect of MultiStem ${ }^{\circledR}$ treatment compared to placebo. Furthermore, post-hoc analysis demonstrated that earlier treatment was more favorable at both 90 and 365 days follow-up. ${ }^{29,30}$ 
In the current model, we administered MAPC treatment in utero where pulmonary circulation is bypassed by the ductus arteriosus and the foramen ovale. Upon birth these two fetal cardiac shunts are closed due to the need for pulmonary gas exchange. This is accompanied by changes in blood flow. Systemic administration of stem cells during postnatal life poses a major risk for accumulation of cells in the pulmonary capillary bed, possibly leading to pulmonary emboli and subsequent respiratory distress. The smaller size of MAPC cells compared to MSC facilitates passage through the pulmonary capillary bed, thereby reducing the risk of pulmonary complications and increasing availability of MAPC cells in the systemic and cerebral vasculature and thus enhancing their neuroprotective effects. ${ }^{31}$ Furthermore, in accordance to studies demonstrating that MAPC are cleared from the bloodstream within 48 to 72 hours after administration, we did not detect MAPC in brain, spleen or lung tissue 7 days after global $\mathrm{HI}$ (i.e. 3 days (i.e. 3 days after the second dose of MAPC). ${ }^{26,} 32$ These findings indicate that MAPC are cleared in the recipient several days after administration. This implies that potential long term side-effects of cell therapy are reduced to a minimum.

Whereas immune-modulation is a crucial component of the therapeutic effects of MAPC treatment, caution should be taken when administered to preterm infants. Due to an underdeveloped immune system and potential in utero immune challenges, including chorioamnionitis, preterm infants are extremely vulnerable for infections. Alterations to the fetal immune system might lead to temporary immune-suppression and the inability to combat infections. ${ }^{33}$ Alternatively, MSCs have proven to enhance the phagocytic ability of immune cells, including neutrophils, and improve effective pathogen clearance, thereby reducing the severity of sepsis. ${ }^{34}$

Transplanted MSCs exert their protective effects primarily through trophic support. This concept is supported by studies demonstrating comparable therapeutic effects of MSCconditioned medium when compared to its cellular equivalent. ${ }^{35,36}$ In addition to secreting an array of soluble factors, it has also been recognized that MSCs release large numbers of extracellular vesicles (EVs) which are involved in cellular communication as paracrine mediators and possibly transfer trophic factors between cells.

Therefore, in a study described in chapter 3, we investigated the therapeutic effects of intravenous administration of MSC-EVs on HI-induced injury of the preterm brain. Intravenous MSC-EV administration resulted in a reduction of electro cortical seizure burden and preservation of the baroreceptor reflex sensitivity after global $\mathrm{HI}$ and partially protected against structural injury of the preterm brain after global HI without reducing cerebral inflammation. In contrast to previous findings, suggesting that neuroprotection of stromal cell therapy (MSC and MAPC respectively) could, at least in part, be attributed to attenuation of neuroinflammation, MSC-EV administration did not attenuate neuroinflammation. This implicates that in addition to anti-inflammatory mechanisms, inflammation-independent mechanisms play a crucial role in the 
neuroprotective potential of stromal cells. This concept is supported by several studies showing that MSC-EVs establish neuroprotection and regeneration through stimulation of angiogenesis and neurogenesis after hypoxia-ischemia. ${ }^{37-43}$ MSCs are known to secrete a number of neurotrophic and angiogenic factors, induce angiogenesis, neurogenesis and astroglial growth and activation, promote synaptogenesis and therefore enhance synaptic connections and axonal remyelination, and decrease apoptosis. Furthermore, it was recently shown in neuron cultures and mice using models of excitotoxicity that administration of MSCs or MSC-conditioned medium mediated neuroprotection through inhibition of glutamate receptor expression and function. ${ }^{44}$

Despite the observed discrepancies in therapeutic effects between MSCs and MSC-EVs, the application of EVs offers several advantages over the administration of MSCs (table 1): (1) the potential risk of malignant transformation is greatly reduced because EVs are non-self-replicating. (2) Due to their small size, EVs are less likely to generate emboli upon intravenous administration, as may be the case with MSCs. (3) EVs can be sterilized by filtration. Thus, from a regulatory point of view, the production and especially the quality control of EV fractions for clinical treatment application are less complicated compared to a primary cells or in vitro expanded cells. (4) EVs can be developed independently of the original donor (as they are derived from MSCs from unrelated donors) and thus offer the opportunity to be turned into an "off-the-shelf" product. (5) EVs are easier to store, and in the future freeze-dried exosome preparations will be available. ${ }^{45}$ This reduces the logistic burden involved in storage of stem cells in liquid nitrogen and makes the cell product readily available within the short window of therapeutic opportunity after the injurious insult. (6) EVs may more easily cross the blood-brain-barrier, possibly enhancing the protection of the penumbra where the blood-brain-barrier is still intact.

The beneficial effects of MSC treatment cannot be exclusively attributed to release of trophic factors. A study by González et al. demonstrated that macrophages and adipose-derived MSCs remain their IL-10 production only when cell-cell contact is maintained. ${ }^{48}$ Also, stromal cells intrinsically possess immune-modulatory properties that are enhanced by a pro-inflammatory micro environment in vivo. The obtained EVs used for intravenous administration were obtained from unlicensed MSCs. Lacking an own metabolism, these EVs cannot modify their content, which may account for the absence of anti-inflammatory properties. However, controlled in vitro licensing or genetic manipulation of MSCs might enhance the anti-inflammatory potential and contribute to a targeted clinically safe cell-free therapy. ${ }^{49}$ This type of manipulation requires a uniform homogeneous population of stromal cells with stable genetics throughout expansion and manipulations. ${ }^{49}$ These criteria are met by MAPC cells. 
Table 1

\begin{tabular}{|c|c|c|c|}
\hline Comparison & Mesenchymal stem cells ${ }^{1}$ & $\begin{array}{l}\text { Multipotent Adult Progenitor } \\
\text { Cells }^{2}\end{array}$ & $\begin{array}{l}\text { Mesenchymal stem cell- } \\
\text { derived extracellular vesicles }{ }^{3}\end{array}$ \\
\hline \multicolumn{2}{|c|}{$\begin{array}{l}\text { NeuroinflammationAttenuation of inflammation } \\
\qquad \text { (local) }\end{array}$} & $\begin{array}{l}\text { Attenuation of inflammation } \\
\text { (local) }\end{array}$ & No protection \\
\hline Structural injury & White matter protection & White matter protection & No protection \\
\hline \multirow[t]{2}{*}{ Brain function } & \multirow[t]{2}{*}{ Reduced seizure burden } & Reduced seizure burden & Reduced seizure burden \\
\hline & & $\begin{array}{l}\text { Improved baroreceptor reflex } \\
\text { sensitivity }\end{array}$ & $\begin{array}{l}\text { Improved baroreceptor } \\
\text { reflex sensitivity }\end{array}$ \\
\hline Selectivity & $\begin{array}{l}\text { Adjusts to in vivo } \\
\text { microenvironment (licensing) }\end{array}$ & $\begin{array}{l}\text { Adjusts to in vivo } \\
\text { microenvironment (licensing) }\end{array}$ & $\begin{array}{l}\text { Determined by in vitro } \\
\text { condition of ' mother cell' }\end{array}$ \\
\hline \multirow[t]{5}{*}{ Clinical product } & Heterogeneous population & Homogenous population & Sterilized by filtration \\
\hline & \multirow[t]{4}{*}{$\begin{array}{l}\text { Limited expansion pot } \\
\text { Low immunogenicity }\end{array}$} & \multirow[t]{2}{*}{$\begin{array}{l}\text { High expansion potential with } \\
\text { stable genetics } \\
\text { Low immunogenicity }\end{array}$} & \multirow[t]{2}{*}{$\begin{array}{l}\text { Can be developed } \\
\text { independently of the original } \\
\text { donor }\end{array}$} \\
\hline & & & \\
\hline & & $\begin{array}{l}\text { Off-the-shelf product: } \\
\text { MultiStem }{ }^{\circledR}\end{array}$ & $\begin{array}{l}\text { Readily available within time } \\
\text { window of opportunity (i.e. } 6 \\
\text { hours after HI) }\end{array}$ \\
\hline & & $\begin{array}{l}\text { Readily available within time } \\
\text { window of opportunity (i.e. } 6 \\
\text { hours after } \mathrm{HI} \text { ) }\end{array}$ & $\begin{array}{l}\text { Variability from batch to } \\
\text { batch production }\end{array}$ \\
\hline
\end{tabular}

${ }^{1}$ Based on Jellema et al., 2013. ${ }^{17}$

${ }^{2}$ Based on Jellema \& Ophelders et al., $2015{ }^{46}$

${ }^{3}$ Based on Ophelders et al., $2016 .{ }^{47}$

In contrast to $\mathrm{HI}$-induced neuroinflammation, which is a sterile inflammatory reaction in response to a single insult (hypoxia-ischemia respectively), intra-amniotic exposure to Candida albicans (C. albicans), as investigated in chapter 4, caused a persistent systemic immune response with a concomitant microglial and astroglial response, white matter injury, and fetal death within 5 days upon infection, without apparent invasion into the brain parenchyma. A similar pattern of cerebral injury was observed following intraamniotic exposure to endotoxin. Intra-amniotic Fluconazole treatment was successful for eradication of the infectious trigger and temporarily reduced the systemic immune response without attenuation of the neuroinflammatory response and protection against structural injury. Nonetheless, and most importantly, single treatment promoted fetal survival. Collectively, we concluded that inflammation as such, and not the stimulus, to be the most important factor in fetal brain injury within the first days after induction of chorioamnionitis.

Our current findings suggest that timing of the (antifungal) treatment regimen is of great importance as early treatment prevented fetal demise. However, a single dose regimen temporarily reduced the systemic response and failed to prolong this reduction. Therefore, we postulate that a multiple dosing strategy will be beneficial for complete eradication of $C$. albicans and will attenuate the subsequent systemic immune response. As eradication of the fungus and (temporary) reduction of the systemic 
immune response had no effects on cerebral inflammation and structural injury, these data suggest that additional immune modulatory therapy is required to protect the fetal brain.

Cell-based therapies might be such an immune-modulatory therapy. Our previously described studies on the therapeutic effects of cell-based therapies following global hypoxia-ischemia have demonstrated reduction of the detrimental cerebral and peripheral immune reaction, and prevented against white matter injury. ${ }^{17,46,47}$

Besides neuroprotective capacities, cell-based therapies (MSC) have proven therapeutic efficacy in models of necrotizing enterocolitis (NEC) and bronchopulmonary dysplasia (BPD), which are two major complications of intra-uterine infection and subsequent preterm birth $^{4,50-52}$ and are correlated to adverse neurodevelopment. ${ }^{53}$ Mostly inflammation-dependent mechanisms ${ }^{54,55}$ are described, but effects on impaired cerebrovascular autoregulation and subsequent hypoxia-ischemia might play essential roles as well. ${ }^{56}$ Therefore, the immune modulatory effects of MSCs may not only improve intestinal and pulmonary outcomes in NEC and BPD but may also indirectly prevent adverse neurodevelopmental outcomes by preventing secondary cerebral injury and supports a multi-organ approach through systemic administration of MSCs.

Furthermore, several studies have shown beneficial effects of MSCs in various in vitro and in vivo models of sepsis. Human MSCs have shown to attenuate NF-K $\beta$ activation and nuclear translocation during inflammatory conditions. ${ }^{57,58}$ Furthermore, interferon gamma-stimulated MSCs showed broad anti-microbial effector functions. ${ }^{59}$ In vivo models of sepsis confirmed the antimicrobial functions of MSCs, which reduced the number of colony-forming-units and decreased bacterial counts. ${ }^{60-62}$ Mechanisms responsible for the antimicrobial actions of MSCs are attributed to inflammationinduced secretion of antimicrobial proteins (i.e. bacterial defensins, LL-37, lipocalin-3, hepcidin, and keratinocyte growth factor, improvement of phagocytosis activity of blood monocytes, increased circulating neutrophil counts and enhanced neutrophil respiratory bursts. ${ }^{63-67}$

\section{Neuroprotective strategies for perinatal infection}

At present no established therapies to reduce inflammation-related injury of the preterm brain are available. Possible therapies include antibiotics, anti-inflammatory agents, inhibition of cell-death pathways, promotion of cellular maturation, and development, and cellular regeneration/replacement.

Antibiotic treatment has been tested extensively, but demonstrated that prophylactic therapy increased the risk of neonatal death and neurodevelopmental disability. Postnatal treatment with tetracycline (minocycline) treatment has been associated with improved neurodevelopmental outcomes in animal models of intra-uterine 
inflammation. However, due to the risk of disturbed bone and tooth enamel formation, tetracyclines are contraindicated in the current setting of inflammation induced injury of the preterm brain. ${ }^{68}$

Antenatal maternal glucocorticoid administration is routine treatment when preterm labor is pending as they enhance lung maturation, thereby preventing acute neonatal morbidity and mortality. ${ }^{69}$ Mixed reports have been published on the effects of glucocorticoid treatment on neurodevelopmental outcomes. A report by Kuypers et al. demonstrated that prophylactic glucocorticoid treatment prevented adverse neurodevelopmental outcomes, whereas treatment after the onset of intra-uterine inflammation exacerbated injury. ${ }^{6}$ Collectively, these data suggest that glucocorticoid therapy is not beneficial when infection/inflammation is established.

Reactive oxygen species play essential roles in the pathogenesis of brain injury. ${ }^{70,71}$ Melatonin, a naturally occurring circadian hormone with antioxidant and antiinflammatory properties ${ }^{72}$, has proven neuroprotective properties in a piglet model of perinatal asphyxia caused by both hypoxia-ischemia and intra-uterine inflammation. ${ }^{73}$ Moreover, it is already widely used clinically. ${ }^{74}$ Erythropoietin (EPO), which is a hematopoietic hormone that regulates erythrocyte production, has shown to possess anti-inflammatory, anti-oxidant, anti-apoptotic, and neurotrophic properties. ${ }^{75}$ Increasing evidence arises on the neuroprotective properties of EPO in inflammationinduced brain injury. Continuous administration of recombinant EPO during the early recovery phase in an ovine model of global hypoxia-ischemia was neuroprotective and improved electrophysiological and cerebrovascular recovery, in association with reduced apoptosis and inflammation. ${ }^{76}$ Moreover, a recent clinical study by Song et al. demonstrated that repeated administration of a low-dose rhEPO for two weeks significantly reduced the incidence of brain injury, including intra cranial hemorrhage and periventricular leukomalacia, in preterm infants. Besides neuroprotection, rhEPO administration decreased the risk for NEC and sepsis, thereby indicating its wide-spread protective effects. $^{77}$

\section{FUTURE DIRECTIONS}

In this thesis we have demonstrated that immune modulation protects the preterm brain from injury and dysfunction after in utero adverse events (e.g. intra-amniotic infection and hypoxia-ischemia). Within the context of immune modulation, future research should focus on immunological interplay between the central nervous system and the peripheral immune system. 


\section{Dual role of microglia / macrophages}

As described before, microglia are among the first responders to injury of the preterm brain. Moreover, they are among the most potent modulators of CNS repair and regeneration. However, they have dualistic roles in the battle for neurological recovery. On the one hand, microglia activation promotes brain recovery by clearing cell debris, resolving local inflammation, and releasing a plethora of trophic factors. On the other hand, microglia activation hinders CNS repair and contributes to expansion of tissue damage. These opposite functions are reflected in the acquisition of distinct phenotypes in response to different micro environmental signals. Classically activated M1 microglia typically release destructive pro-inflammatory mediators. In contrast, alternatively activated $\mathrm{M} 2$ phenotypes release numerous protective/ trophic factors. In particular the $\mathrm{M} 2$ phenotype is known to promote regenerative processes including neurogenesis, axonal remodeling, angiogenesis, oligodendrogenesis, and remyelination. Considering these adaptive functions, treatment strategies should be aimed at modulation of neuroinflammation instead of suppression. MSCs and MAPC cells have proven capacities to drive microglia polarization in vitro and in vivo. ${ }^{24,78}$ However, the effects on microglia/macrophage polarization in our models remain to be elucidated and proper diagnosis of intra-uterine infection and/or hypoxia-ischemia without apparent life threatening fetal distress is mandatory.

Recent studies in CNS injury models show that the majority of newly recruited microglia/macrophages at the site of injury express M2 signature genes whereas microglia expressing M1 genes dominate the site of injury about 1 week after injury. ${ }^{79-81}$ This phenotype switch from M2-dominant to M1-dominant may result from a M2-to M1 conversion within activated microglia, as well as the recruitment of proinflammatory M1 microglia/macrophages to the site of injury. ${ }^{82}$ In addition, although both white and grey matter exhibit M2-to-M1 switch after injury, the temporal dynamics of the switch differ between the two types of tissue and more prominent phenotypic changes occur in white matter. ${ }^{82}$ The temporal dynamics of microglia phenotypical switch indicate that timing of treatment is of crucial importance since prevention of M1 microglia as the prevailing phenotype might act as therapeutic target. Moreover, in vivo studies using bone marrow chimeras to distinguish microglia from bone marrow-derived macrophages have confirmed that resident microglia and bone marrow-derived macrophages are both involved in phenotype switching. ${ }^{83,84}$ Moreover, studies have shown that monocytes can be primed towards an immune modulatory M2 phenotype through exposure to mesenchymal stromal cells prior licensed to exert anti-

inflammatory functions. ${ }^{85,86}$ As such, we postulate a role for monocytes as therapeutic target. We hypothesize that in vivo education of monocytes by MSCs or MSC-EVs and subsequent differentiation into alternatively activated macrophages might contribute to attenuation of neuroinflammation and promote regeneration. Within this concept, the 
spleen, as a reservoir for immune-effector cells, is of crucial importance since splenic monocytes, in contrast to bone marrow and peripheral monocytes, have demonstrated to generate alternatively activated macrophages following exposure to $\mathrm{MSCs}^{87}$

Furthermore, targeting peripheral immune effector cells to exert neuroprotective functions would implicate that homing of stem cells in the cerebral vasculature and parenchyma is no prerequisite for neuroprotective actions and justifies intravenous administration, as performed in this thesis. Collectively, this paves the road for modulation of the fetal immune system following in utero ischemic compromise through maternal administration of stem cells. Recently, maternally administered MSCS modulated the maternal immune system and the fetal immune response following intra-amniotic endotoxin exposure. Interestingly, these maternally administered MSCs protected against preterm birth, brain injury and motor deficits. ${ }^{88}$

As proposed in chapter 3, enhancing the anti-inflammatory potential of MSC-EVs through inflammatory licensing might improve their therapeutic efficacy. Also, understanding the temporal dynamics of injury and repair might reveal specific phases that require enhancement of processes other than anti-inflammatory, such as angiogenesis. Generation of EVs that specifically target these processes can contribute to neuroprotection. Moreover, as suggested in chapter 4, exerting a synergistic approach in which cell-based therapies are combined with therapeutic approaches, such as EPO, melatonin, glucocorticoids, and hypothermia, might improve neurodevelopmental outcomes. Such a synergy has been demonstrated in an animal model of BPD where combined treatment with MSCs and erythropoietin resulted in more promotion of lung repair compared to MSCs or EPO treatment alone. ${ }^{89}$

In conclusion, the work described in this thesis demonstrated the promising effects of immune-modulatory therapies in the protection of the preterm brain after intraamniotic infection and hypoxia-ischemia, which all activate the immune system. Temporal dynamics of peripheral immune effector cell mobilization and subsequent cerebral infiltration might therefore provide information crucial for timing of cell-based, cell-derived or anti-microbial therapies. Furthermore, the potential clinical impact and mechanisms of action of these promising therapies needs to be further assessed in long-term follow-up studies in appropriate animal models. 


\section{REFERENCES}

1. Fleiss, B. et al. Inflammation-induced sensitization of the brain in term infants. Developmental Medicine \& Child Neurology 57, 17-28 (2015).

2. Beck, S. et al. The worldwide incidence of preterm birth: a systematic review of maternal mortality and morbidity. Bull World Health Organ 88, 31-38 (2010).

3. Jellema, R.K. et al. Cerebral inflammation and mobilization of the peripheral immune system following global hypoxia-ischemia in preterm sheep. J Neuroinflammation 10, 2094-2010 (2013).

4. Gantert, M. et al. Chorioamnionitis: a multiorgan disease of the fetus? J Perinatol 30 Suppl, S21-30 (2010).

5. Back, S.A., Riddle, A., Dean, J. \& Hohimer, A.R. The instrumented fetal sheep as a model of cerebral white matter injury in the premature infant. Neurotherapeutics : the journal of the American Society for Experimental NeuroTherapeutics 9, 359-370 (2012).

6. Kuypers, E. et al. Effects of intra-amniotic lipopolysaccharide and maternal betamethasone on brain inflammation in fetal sheep. PLoS One 8, e81644 (2013).

7. Gunn, A.J. \& Bennet, L. Fetal hypoxia insults and patterns of brain injury: insights from animal models. Clin Perinatol 36, 579-593 (2009)

8. Bennet, L. et al. Cell therapy for neonatal hypoxia-ischemia and cerebral palsy. Annals of neurology 71, 589-600 (2012).

9. Titomanlio, L. et al. Stem cell therapy for neonatal brain injury: perspectives and challenges. Annals of neurology 70, 698-712 (2011).

10. van Velthoven, C.T., Kavelaars, A., van Bel, F. \& Heijnen, C.J. Regeneration of the ischemic brain by engineered stem cells: fuelling endogenous repair processes. Brain research reviews 61, 1-13 (2009).

11. Gortner, L. et al. Regenerative therapies in neonatology: clinical perspectives. Klinische Pädiatrie 224, 233 (2012).

12. Borlongan, C.V. \& Weiss, M.D. Baby STEPS: a giant leap for cell therapy in neonatal brain injury. Pediatr Res 70, 3-9 (2011).

13. Donega, V., van Velthoven, C.T., Nijboer, C.H., Kavelaars, A. \& Heijnen, C.J. The endogenous regenerative capacity of the damaged newborn brain: boosting neurogenesis with mesenchymal stem cell treatment. Journal of Cerebral Blood Flow \& Metabolism 33, 625-634 (2013).

14. van Velthoven, C.T., Kavelaars, A. \& Heijnen, C.J. Mesenchymal stem cells as a treatment for neonatal ischemic brain damage. Pediatr Res 71, 474-481 (2012).

15. Dalous, J. et al. Use of human umbilical cord blood mononuclear cells to prevent perinatal brain injury: a preclinical study. Stem cells and development 22, 169-179 (2012).

16. Fleiss, B. et al. Stem cell therapy for neonatal brain injury. Clinics in perinatology 41, 133-148 (2014).

17. Jellema, R.K. et al. Mesenchymal stem cells induce T-cell tolerance and protect the preterm brain after global hypoxia-ischemia. (2013).

18. Jacobs, S.A., Roobrouck, V.D., Verfaillie, C.M. \& Van Gool, S.W. Immunological characteristics of human mesenchymal stem cells and multipotent adult progenitor cells. Immunol Cell Biol 91, 32-39 (2013).

19. Boozer, S. et al. Global Characterization and Genomic Stability of Human MultiStem, A Multipotent Adult Progenitor Cell. Journal of stem cells 4, 17-28 (2008).

20. Jacobs, S.A. et al. Human multipotent adult progenitor cells are nonimmunogenic and exert potent immunomodulatory effects on alloreactive T-cell responses. Cell Transplant 22, 1915-1928 (2013).

21. Kovacsovics-Bankowski, M. et al. Pre-clinical safety testing supporting clinical use of allogeneic multipotent adult progenitor cells. Cytotherapy 10, 730-742 (2008).

22. Bedi, S.S. et al. Intravenous multipotent adult progenitor cell therapy attenuates activated microglial/macrophage response and improves spatial learning after traumatic brain injury. Stem Cells Trans/ Med 2, 953-960 (2013).

23. Busch, S.A. et al. Multipotent adult progenitor cells prevent macrophage-mediated axonal dieback and promote regrowth after spinal cord injury. The Journal of Neuroscience 31, 944-953 (2011). 
24. Walker, P.A. et al. Intravenous multipotent adult progenitor cell therapy after traumatic brain injury: modulation of the resident microglia population. J neuroinflammation 9, 228 (2012).

25. Walker, P.A. et al. Intravenous multipotent adult progenitor cell therapy for traumatic brain injury: preserving the blood brain barrier via an interaction with splenocytes. Exp Neurol 225, 341-352 (2010).

26. Yasuhara, T. et al. Intravenous grafts recapitulate the neurorestoration afforded by intracerebrally delivered multipotent adult progenitor cells in neonatal hypoxic-ischemic rats. Journal of Cerebral Blood Flow and Metabolism 28, 1804-1810 (2008).

27. DePaul, M.A. et al. Intravenous multipotent adult progenitor cell treatment decreases inflammation leading to functional recovery following spinal cord injury. Sci Rep 5, 16795 (2015).

28. Mays, R. \& Deans, R. Adult adherent cell therapy for ischemic stroke: clinical results and development experience using MultiStem. Transfusion 56, 6S-8S (2016).

29. inc., A. One-Year Results From Phase 2 Stroke Study of MultiStem ${ }^{\circledR}$ Cell Therapy Demonstrate a Significantly Higher Rate of Complete or Nearly Full Recovery. 2016.

30. Hess, D.C. et al. Final Results of the B01-02 Phase 2 Trial Testing the Safety and Efficacy of MultiStem ${ }^{\circledR}$ in Treatment of Ischemic Stroke. Stroke 47, A71-A71 (2016).

31. Fischer, U.M. et al. Pulmonary passage is a major obstacle for intravenous stem cell delivery: the pulmonary first-pass effect. Stem cells and development 18, 683-692 (2009).

32. Mays, R.W. et al. Development of an allogeneic adherent stem cell therapy for treatment of ischemic stroke. J Exp Stroke Transl Med 3, 34-46 (2010).

33. Wolfs, T.G. et al. Inflammation-induced immune suppression of the fetus: a potential link between chorioamnionitis and postnatal early onset sepsis. The Journal of Maternal-Fetal \& Neonatal Medicine 25, 8-11 (2012).

34. Kingsley, S.M.K. \& Bhat, B.V. Could stem cells be the future therapy for sepsis? Blood Reviews (2016).

35. Lai, R.C., Chen, T.S. \& Lim, S.K. Mesenchymal stem cell exosome: a novel stem cell-based therapy for cardiovascular disease. Regenerative medicine 6, 481-492 (2011).

36. Lai, R.C. et al. Exosome secreted by MSC reduces myocardial ischemia/reperfusion injury. Stem cell research 4, 214-222 (2010).

37. Salomon, C. et al. Exosomal signaling during hypoxia mediates microvascular endothelial cell migration and vasculogenesis. PLoS One 8, e68451 (2013).

38. Bian, S. et al. Extracellular vesicles derived from human bone marrow mesenchymal stem cells promote angiogenesis in a rat myocardial infarction model. J Mol Med (Berl) 92, 387-397 (2014).

39. Zhang, B. et al. Human umbilical cord mesenchymal stem cell exosomes enhance angiogenesis through the Wnt4/beta-catenin pathway. Stem Cells Trans/ Med 4, 513-522 (2015).

40. Chen, J. et al. Proangiogenic compositions of microvesicles derived from human umbilical cord mesenchymal stem cells. PLoS One 9, e115316 (2014).

41. Xin, H. et al. Systemic administration of exosomes released from mesenchymal stromal cells promote functional recovery and neurovascular plasticity after stroke in rats. J Cereb Blood Flow Metab 33, 17111715 (2013).

42. Döppner, T.R. et al. Extracellular vesicles improve post-stroke neuroregeneration and prevent postischemic immunosuppression. Stem cells translational medicine (in press).

43. Zhang, Y. et al. Effect of exosomes derived from multipluripotent mesenchymal stromal cells on functional recovery and neurovascular plasticity in rats after traumatic brain injury. Journal of neurosurgery $122,856-867$ (2015).

44. Voulgari-Kokota, A. et al. Mesenchymal stem cells protect CNS neurons against glutamate excitotoxicity by inhibiting glutamate receptor expression and function. Experimental neurology 236, 161-170 (2012).

45. Method for preparing exosome freeze-dried powder of human amniotic mesenchymal stem cells. Google Patents; 2015.

46. Jellema, R.K. et al. Multipotent adult progenitor cells for hypoxic-ischemic injury in the preterm brain. Journal of neuroinflammation 12, 241 (2015).

47. Ophelders, D. et al. Mesenchymal stromal cellderived extracellular vesicles protect the fetal brain after hypoxia-ischemia. Stem cells translational medicine 5, 754-763 (2016). 


\section{Chapter 5}

48. González, M.A., Gonzalez-Rey, E., Rico, L., Büscher, D. \& Delgado, M. Adipose-derived mesenchymal stem cells alleviate experimental colitis by inhibiting inflammatory and autoimmune responses. Gastroenterology 136, 978-989 (2009).

49. Ferguson, S.W. \& Nguyen, J. Exosomes as therapeutics: The implications of molecular composition and exosomal heterogeneity. Journal of Controlled Release 228, 179-190 (2016).

50. Mueller, M. et al. Mesenchymal stem/stromal cells-a key mediator for regeneration after perinatal morbidity? Molecular and cellular pediatrics 3, 1 (2016).

51. Laube, M., Stolzing, A., Thome, U.H. \& Fabian, C. Therapeutic potential of mesenchymal stem cells for pulmonary complications associated with preterm birth. The international journal of biochemistry \& cell biology 74, 18-32 (2016).

52. Pierro, M. \& Thébaud, B. MSCS in Scenarios of Infection and Inflammation: Focus on Neonatal Diseases. Current Stem Cell Reports 2, 158-167 (2016).

53. Dammann, O., Leviton, A., Bartels, D.B. \& Dammann, C.E. Lung and brain damage in preterm newborns. Neonatology 85, 305-313 (2004).

54. Volpe, J.J. Postnatal sepsis, necrotizing entercolitis, and the critical role of systemic inflammation in white matter injury in premature infants. The Journal of pediatrics 153, 160 (2008).

55. Shah, D.K. et al. Adverse neurodevelopment in preterm infants with postnatal sepsis or necrotizing enterocolitis is mediated by white matter abnormalities on magnetic resonance imaging at term. The Journal of pediatrics 153, 170-175. e171 (2008).

56. Schat, T.E. et al. Assessing cerebrovascular autoregulation in infants with necrotizing enterocolitis using near-infrared spectroscopy. Pediatr Res 79, 76-80 (2015).

57. Qiu, J., Li, W., Feng, S., Wang, M. \& He, Z. Transplantation of bone marrow-derived endothelial progenitor cells attenuates cerebral ischemia and reperfusion injury by inhibiting neuronal apoptosis, oxidative stress and nuclear factor-kB expression. International journal of molecular medicine 31, 91-98 (2013).

58. Choi, H., Lee, R.H., Bazhanov, N., Oh, J.Y. \& Prockop, D.J. Anti-inflammatory protein TSG-6 secreted by activated MSCs attenuates zymosan-induced mouse peritonitis by decreasing TLR2/NF-KB signaling in resident macrophages. Blood 118, 330-338 (2011).

59. Meisel, R. et al. Human but not murine multipotent mesenchymal stromal cells exhibit broad-spectrum antimicrobial effector function mediated by indoleamine 2, 3-dioxygenase. Leukemia 25, 648-654 (2011).

60. Hall, S.R. et al. Mesenchymal Stromal Cells Improve Survival During Sepsis in the Absence of Heme Oxygenase-1: The Importance of Neutrophils. Stem Cells 31, 397-407 (2013).

61. Krasnodembskaya, A. et al. Human mesenchymal stem cells reduce mortality and bacteremia in gramnegative sepsis in mice in part by enhancing the phagocytic activity of blood monocytes. American Journal of Physiology-Lung Cellular and Molecular Physiology 302, L1003-L1013 (2012).

62. Yuan, Y. et al. Marrow mesenchymal stromal cells reduce methicillin-resistant Staphylococcus aureus infection in rat models. Cytotherapy 16, 56-63 (2014).

63. Alcayaga-Miranda, F. et al. Combination therapy of menstrual derived mesenchymal stem cells and antibiotics ameliorates survival in sepsis. Stem cell research \& therapy 6, 1 (2015).

64. Sung, D.K. et al. Antibacterial effect of mesenchymal stem cells against Escherichia coli is mediated by secretion of beta-defensin-2 via toll-like receptor 4 signalling. Cellular microbiology (2015).

65. Gupta, N. et al. Mesenchymal stem cells enhance survival and bacterial clearance in murine Escherichia coli pneumonia. Thorax, thoraxjnl-2011-201176 (2012).

66. Krasnodembskaya, A. et al. Antibacterial Effect of Human Mesenchymal Stem Cells Is Mediated in Part from Secretion of the Antimicrobial Peptide LL-37. Stem cells 28, 2229-2238 (2010).

67. Lee, J.W. et al. Therapeutic effects of human mesenchymal stem cells in ex vivo human lungs injured with live bacteria. American journal of respiratory and critical care medicine 187, 751-760 (2013).

68. Been, J., Degraeuwe, P., Kramer, B. \& Zimmermann, L. Antenatal steroids and neonatal outcome after chorioamnionitis: a meta-analysis. BJOG: An International Journal of Obstetrics \& Gynaecology 118, 113122 (2011). 
69. Ranchhod, S. et al. Potential neuroprotective strategies for perinatal infection and inflammation. International Journal of Developmental Neuroscience 45, 44-54 (2015).

70. Back, S.A. et al. Selective vulnerability of preterm white matter to oxidative damage defined by F2isoprostanes. Annals of neurology 58, 108-120 (2005).

71. Baburamani, A.A., Supramaniam, V.G., Hagberg, H. \& Mallard, C. Microglia toxicity in preterm brain injury. Reproductive Toxicology 48, 106-112 (2014).

72. Reiter, R.J., Calvo, J.R., Karbownik, M., Qi, W. \& Tan, D.X. Melatonin and its relation to the immune system and inflammation. Annals of the New York Academy of Sciences 917, 376-386 (2000).

73. Robertson, N.J. et al. Melatonin augments hypothermic neuroprotection in a perinatal asphyxia model. Brain 136, 90-105 (2013).

74. Chen, Y.-C., Tain, Y.-L., Sheen, J.-M. \& Huang, L.-T. Melatonin utility in neonates and children. Journal of the Formosan Medical Association 111, 57-66 (2012).

75. Robertson, N.J. et al. Which neuroprotective agents are ready for bench to bedside translation in the newborn infant? The Journal of pediatrics 160, 544 (2012).

76. Wassink, G. et al. Partial white and grey matter protection with prolonged infusion of recombinant human erythropoietin after asphyxia in preterm fetal sheep. Journal of Cerebral Blood Flow \& Metabolism, 0271678X16650455 (2016).

77. Song, J. et al. Recombinant human erythropoietin improves neurological outcomes in very preterm infants. Annals of neurology (2016).

78. Zanier, E.R. et al. Bone marrow mesenchymal stromal cells drive protective M2 microglia polarization after brain trauma. Neurotherapeutics 11, 679-695 (2014).

79. Perego, C., Fumagalli, S. \& De Simoni, M.G. Temporal pattern of expression and colocalization of microglia/macrophage phenotype markers following brain ischemic injury in mice. J Neuroinflammation 8, 174 (2011).

80. Hu, X. et al. Microglia/macrophage polarization dynamics reveal novel mechanism of injury expansion after focal cerebral ischemia. Stroke 43, 3063-3070 (2012).

81. Wang, G. et al. Microglia/macrophage polarization dynamics in white matter after traumatic brain injury. J Cereb Blood Flow Metab 33, 1864-1874 (2013).

82. Hu, X. et al. Microglial and macrophage polarization [mdash] new prospects for brain repair. Nature Reviews Neurology 11, 56-64 (2015).

83. Wattananit, S. et al. Monocyte-derived macrophages contribute to spontaneous long-term functional recovery after stroke in mice. Journal of Neuroscience 36, 4182-4195 (2016).

84. Shechter, R. \& Schwartz, M. Harnessing monocyte-derived macrophages to control central nervous system pathologies: no longer 'if'but 'how'. The Journal of pathology 229, 332-346 (2013).

85. Melief, S.M. et al. Multipotent stromal cells induce human regulatory T cells through a novel pathway involving skewing of monocytes toward anti-inflammatory macrophages. Stem Cells 31, 1980-1991 (2013).

86. Zeuner, M.-T., Patel, K., Denecke, B., Giebel, B. \& Widera, D. Paracrine effects of TLR4-polarised mesenchymal stromal cells are mediated by extracellular vesicles. Journal of translational medicine 14, 34 (2016)

87. Le Blanc, K. \& Davies, L.C. Mesenchymal stromal cells and the innate immune response. Immunology letters 168, 140-146 (2015).

88. Lei, J. et al. Murine model: maternal administration of stem cells for prevention of prematurity. American journal of obstetrics and gynecology 212, 639. e631-639. e610 (2015).

89. Luan, Y. et al. Mesenchymal stem cells in combination with erythropoietin repair hyperoxia-induced alveoli dysplasia injury in neonatal mice via inhibition of TGF- $\beta 1$ signaling. Oncotarget (2016). 

Summary 

Brain damage is a major contributor to mortality and morbidity in preterm infants and is mostly caused by lack of oxygen (hypoxia-ischemia) and / or intra-amniotic infection (chorioamnionitis). At present there is no cure for brain damage in this vulnerable group of children and current therapies are aimed at treatment of symptoms and stabilizing vital functions. It is, therefore, important that new treatment strategies be developed for the protection of the premature brain.

The aim of this thesis was to test new therapies for brain damage caused by oxygen deprivation or treat an amniotic fluid infection in preterm infants.

\section{CELL-BASED THERAPY}

Systemic and local immune activation are causally involved in the pathophysiology of brain injury, regardless of its cause. However, activation of immune cells is also necessary for regeneration of injured tissue and an imbalance in these two properties leads to brain injury in preterm infants.

Therefore, we hypothesized that therapeutic strategies should possess (1) anti-inflammatory and (2) regenerative properties. Stem cells meet both of these characteristics.

In chapter 2 we investigated the neuroprotective effects of Multipotent Adult Progenitor Cells (MAPCs), which are bone marrow-derived stromal cells with strong anti-inflammatory and regenerative capabilities, in our preclinical ovine model of hypoxia-ischemia.

Administration of MAPCs improved brain function by reducing the number and duration of convulsions. Also, blood pressure regulation in the brainstem was protected.

In addition to functional protection, administration of MAPCs also resulted in a reduced neuroinflammatory response and prevented damage to the cerebral white matter, which is characteristic for brain injury in premature babies).

The mechanisms by which bone marrow-derived stromal cells exert their immune modulatory actions are unclear, but paracrine mechanisms, mediated in part through release of extracellular vesicles (EVs) are suggested to be responsible for these effects.

In chapter 3, we therefore, investigated the protective effects of mesenchymal stromal cell-derived extracellular vesicles (MSC-EVs) in the same preclinical model of preterm hypoxic-ischemic brain injury. Systemic administration of MSC-EVs improved brain function by reducing the total number and duration of seizures, and by preserving baroreceptor reflex sensitivity. These functional protections were associated with a tendency to prevent hypomyelination. Cerebral inflammation remained unaffected by the MSC-EV treatment. 


\section{ANTIMICROBIAL TREATMENT}

In chapter 4 we investigated the effects of intra-amniotic Candida albicans (C. albicans) infection on the preterm brain. Furthermore, we investigated the effects of reducing the fungal load by intra-amniotic antifungal treatment with Fluconazole.

Intra-amniotic exposure to C. albicans caused a severe systemic inflammatory response, illustrated by a robust increase of plasma IL- 6 concentrations. Cerebrospinal fluid cultures were positive for Candida albicans in the majority of the group of 3 day $C$. albicans-exposed animals whereas no positive cultures were present in the group of 5 day C. albicans-exposed and Fluconazole treated animals. C. albicans was not detected in the brain parenchyma. A neuroinflammatory response in the hippocampus and white matter was however detected which was characterized by increased microglial and astrocyte activation. The neuroinflammatory changes were accompanied by structural white matter injury. Intra-amniotic Fluconazole reduced fetal mortality, but did not attenuate either neuroinflammation or white matter injury.

In summary, this thesis demonstrated that modulation, rather than attenuation, of immune reactions with cell-based or anti-microbial treatment protected against injury of the preterm brain following global hypoxia-ischemia or intra-uterine infection. The therapies tested in this thesis show great promise and will form the basis of additional research and clinical trials, leading to improved neurodevelopmental outcomes after $\mathrm{HI}$ or intra-uterine infection. 
Samenvatting 

Hersenschade heeft een belangrijke bijdrage aan de sterfte en morbiditeit bij vroeggeboren kinderen en wordt voornamelijk veroorzaakt door zuurstoftekort (hypoxieischemie) en / of een vruchtwaterinfectie (chorioamnionitis). Op dit moment is er geen behandeling voor hersenschade in deze kwetsbare groep kinderen. Huidige therapieën zijn dan ook gericht op het behandelen van de symptomen en het stabiliseren van de vitale functies. Het is daarom belangrijk dat nieuwe behandelingsstrategieën voor de bescherming van het brein van vroeggeborenen worden ontwikkeld.

Het doel van dit proefschrift was het testen van nieuwe therapieën om hersenschade, veroorzaakt door zuurstoftekort of een vruchtwaterinfectie, in vroeggeboren kinderen te behandelen.

\section{STAMCELTHERAPIE}

Activatie van immuun cellen zowel in als buiten het brein is betrokken bij de ontwikkeling van hersenschade. Echter, deze ontstekingsreactie is ook nodig om beschadigd weefsel te herstellen. Een dysbalans in deze twee eigenschappen leidt ertoe dat hersenschade in vroeggeboren kinderen niet herstelt.

Daarom hypothetiseerden wij dat een therapie de (1) de ontstekingsreactie moet remmen (anti-inflammatoir) en (2) weefselherstel moet stimuleren. Stamcellen voldoen aan beide kenmerken.

In hoofdstuk 2 onderzochten we daarom de beschermende effecten van Multipotent Adult Progenitor Cells (MAPCs) op hersenschade na zuurstof tekort. Dit onderzochten wij in een translationeel diermodel voor premature breinschade ontstaan door zuurstoftekort. MAPCs zijn stamcellen uit het beenmerg met sterke ontstekingsremmende en weefselherstellende capaciteiten.

Toediening van MAPCs verbeterde de hersenfunctie door het aantal en de duur van convulsies te verminderen. Tevens werd de regulatie van de bloeddruk, welke geregeld wordt vanuit de hersenstam, beschermd. Naast functionele bescherming, resulteerde toediening van MAPCs ook in een verminderde ontstekingsreactie in het brein en werd schade aan de witte stof (kenmerkend voor hersenschade in vroeggeborenen) voorkomen.

De mechanismen waarop stamcellen het immuun systeem kunnen beïnvloeden zijn grotendeels onbekend. Een voorgesteld mechanisme is door uitscheiding van extracellulaire (EVs) waarin immuun modulerende moleculen verpakt zitten. Om de bijdrage van EVs aan de beschermende effecten van stamcellen op het premature brein te onderzoeken hebben we in hoofdstuk 3 de effecten van extracellulaire vesikels uit mesenchymale stamcellen (MSC-EV) na zuurstof tekort onderzocht in ons translationeel diermodel 
Toediening van MSC-EVs verbeterde de hersenfunctie door vermindering van het totale aantal en de duur van de convulsies, en door het behoud van bloeddruk regulatie vanuit de hersenstam. Deze functionele bescherming werd geassocieerd met een neiging om witte stof schade te voorkomen voorkomen. De ontstekingsreactie in het brein werd niet verbeterd door behandeling met MSC-EVs.

\section{ANTIMICROBIËLE BEHANDELING}

In hoofdstuk 4 onderzochten we de effecten van een vruchtwaterinfectie met Candida albicans (C. albicans) op het premature brein. Tevens onderzochten we welk effect een eenmalige toediening van anti-schimmel medicatie (Fluconazol) in het vruchtwater had op hersenschade.

Een vruchtwaterinfectie met $C$. albicans veroorzaakt een ernstige systemische ontstekingsreactie in de foetus, gekenmerkt door een sterke toename van de IL-6 concentratie in het bloed. Daarnaast werd C. albicans na drie dagen terug gevonden in de hersenvloeistof (cerebrospinale vloeistof) van de foetus en leidde het tot foetale dood 5 dagen na infectie. Daarnaast veroorzaakte de vruchtwaterinfectie een ontstekingsreactie in het foetale brein die gepaard ging met schade aan de witte stof.

Behandeling met Fluconazol voorkwam foetale dood en verminderde de aanwezigheid van C. albicans in de hersenvloeistof en het hersenweefsel 5 dagen na de infectie. Ook de ontstekingsreactie in het bloed werd tijdelijk geremd. Echter, de ontstekingsreactie en schade aan het premature brein werden niet voorkomen.

Samengevat, in dit proefschrift hebben we aangetoond dat sturing van immuun reacties, in plaats van remming, met stamcellen of antimicrobiële behandeling beschermend kunnen werken tegen hersenschade bij vroeggeboren kinderen. De geteste therapieën in dit proefschrift zijn veelbelovend en zullen de basis vormen voor toekomstig onderzoek en klinische studies leidend tot een behandeling voor hersenschade na zuurstoftekort, vruchtwaterinfecties en vroeggeboorte. 
Valorization 

Preterm birth is the leading cause of perinatal morbidity and mortality in developed countries. In the Netherlands $7.7 \%$ of all neonates are born preterm (less than 37 weeks gestational age) of which $20 \%$ are born extremely preterm (less than 32 weeks gestational age). Although survival after preterm birth has increased in the last decades, still a large proportion of preterm infants suffer from long term morbidity and disability, which have a tremendous impact on patients and their families.

Brain injury is the biggest contributor to mortality and morbidity in preterm infants. 25$50 \%$ of preterm infants suffer from cognitive, socialization, attentional and/or behavioral disorders, whereas, 5-10\% suffer from spastic motoric deficits (e.g. cerebral palsy). Current treatment strategies are focused on limiting secondary injury after hypoxia-ischemia by hypothermia treatment in term babies or treating symptoms and stabilizing vital signs in preterm babies. Infections are treated with anti-microbial agents who are aimed at the microorganisms but not at the injured brain.

Improvement of neurodevelopmental outcomes of this vulnerable group of patients would markedly decrease the burden for this vulnerable patient group. Therefore, the aim of the current thesis was to investigate therapeutic strategies for the prevention of injury of the preterm brain caused by infectious (intra-uterine C. albicans infection) and sterile inflammatory triggers (global hypoxia-ischemia). For this purpose we used a translational ovine model of preterm brain injury caused by either global hypoxiaischemia or intra-uterine infection. We used ovine fetuses since ovine fetal development and physiology are similar to the human situation. Furthermore, the size of the fetus allows for chronic instrumentations and the long gestational period enables us to study specific developmental processes in detail allowing us to apply our therapies at specific time-points.

In this thesis we demonstrated that systemic administration of Multipotent Adult Progenitor Cells (MAPCs) protected the fetal brain following global hypoxia-ischemia. Especially preterm infants suffering from global HI might benefit from MAPC treatment, since early interventions (within 6 hours following global $\mathrm{HI}$ ) are mandated. Autologous transplantation of sufficient numbers of bone marrow stromal cells is time-consuming and exceeds the therapeutic window of opportunity. MAPC treatment has already been translated into a FDA-approved clinical-grade product, named MultiStem (Athersys Inc.), and is therefore already available "off-the-shelf".

Currently, MultiStem is tested extensively in phase 2 clinical trials of ischemic stroke, and preclinical trials traumatic brain injury, multiple sclerosis, and spinal cord injury. Besides neurological indications, other diseases such as cardiovascular, and inflammatory and immune conditions benefit from MAPC treatment. The results in this thesis contribute to the addition of new group of patient, namely preterm infants.

The therapeutic effects of MAPC are mostly attributed to anti-inflammatory properties. The origin of many complications of preterm birth can be narrowed down to 
inflammation, both in utero and postnatally. Therefore, the application of MAPC might reach further than neuroprotection. Future studies will investigate the therapeutic efficacy of MAPC treatment in other neonatal diseases such as bronchopulmonary dysplasia and necrotizing enterocolitis.

Besides anti-inflammatory properties, regenerative properties are also assigned to the therapeutic potential of MAPC cells. This paves the way for MAPC treatment as a secondary treatment in case of injury caused with an infectious origin, as proposed in chapter 4 in which we demonstrated that anti-microbial treatment reduced systemic inflammation, but could not protect against injury of the preterm brain.

As the therapeutic potential of stem cells is mostly achieved by secretion of paracrine factors, packed in extracellular vesicles, we assessed the therapeutic potential of systemic administration of these vesicles, rather than administration of the complete cell product. Administration of EVs poses advantages over administration of cells as (1) the risk of malignant transformation is greatly reduced because EVs are non-selfreplicating (51). (2) Lacking an own metabolism, the EVs activity can hardly be influenced by the in vivo environment in patients, thus allowing for a much better characterization of their functional properties. (3) Owing to their small size EVs are less likely to generate emboli upon intravenous administration, as may be the case with MSCs. (4) In addition, EVs can be sterilized by filtration. Thus, from a regulatory point of view, the production and especially the quality control of EV fractions for clinical treatment application is less complicated than for a cellular therapeutic of in vitro expanded cells (52). (5) Last but not least, EVs can be developed independently of the original donor as they are derived from MSCs from unrelated donors and thus offer the opportunity to be turned into an "off-the-shelf" product. MAPC cells are ideal candidates for an off-the-shelf cell-based product and might provide the source for a superior cell-free product as well.

The ability of cells to adjust their secretome in vivo in reaction to their microenvironment appears to be crucial for the efficacy of cell-based therapy. Due to a lack of a metabolism, EV therapy is static, and the therapeutic properties of the EVs are determined by the in vitro conditions of the donor cell. Since in vitro conditions can be altered, creation of EVs with specific therapeutic properties is possible. Moreover, with the genetic stability of MAPCs, uniform cellular responses upon in vitro stimulation might be expected and reflected in the generation of EVs with specific therapeutic capacities (i.e. immune-modulatory, regenerative), that can be administered off-theshelf at specific time-points. Determination of these time-points requires specific biomarkers, for which our translational ovine model is well-suited.

In conclusion, the therapies tested in this thesis show great promise and form the basis of additional research and clinical trials, leading to improved neurodevelopmental outcomes, and, ultimately, increased disability-free lives. 
Acknowledgements 

Met grote trots en voldoening, maar bovenal met grote dankbaarheid kan ik eindelijk zeggen dat mijn boekje af is. Uiteraard heb ik deze prestatie niet alleen geleverd en wil ik iedereen bedanken die mij op welke wijze dan ook heeft gesteund gedurende mijn promotietraject.

Allereerst wil ik mijn promotieteam, Dr. Tim Wolfs en Dr. Reint Jellema, en Prof. Dr. Boris Kramer heel hartelijk bedanken. Beste Tim, jouw aanstekelijke enthousiasme en doorzettingsvermogen zijn bewonderingswaardig. Dank je wel voor je vertrouwen, morele steun, de 'messcherpe' discussies, en dat je plezier hebt terug gebracht in mijn werk. Ik kijk uit naar onze samenwerking de komende drie jaren. Beste Reint, dankzij jou heb ik als student kennis mogen maken met het kindergeneeskunde onderzoek. Ik herinner mij nog de momenten op het CPV waarin we (soms tot laat) instrumenteerden, occludeerden of elektrodes soldeerden. Jouw ondersteuning heeft mij een enorme duw in de rug gegeven. Dank je wel hiervoor. Beste Boris, dank je wel voor deze unieke kans. Dankzij onze bezoekjes aan de NICU ben ik de patiënt, degene waar we dit toch voor doen, nooit uit het oog verloren.

Ook wil ik graag Prof. Dr. Harry Steinbusch en Prof. Dr. Luc Zimmermann bedanken. Beste Harry en Luc, dank jullie wel dat ik binnen MHeNS en Kindergeeskunde mijn project uit mocht voeren en voor jullie interesse en feedback op mijn projecten.

De leden van de leescommissie bestaande uit Prof. Dr. Yasin Temel (voorzitter), Prof. Dr. Chris Reutelingsperger, Prof. Dr. Niels Hellings, Dr. Bobbi Fleiss en Dr. Jeroen Vermeulen en de leden van de corona wil ik hartelijk danken voor het lezen en beoordelen van mijn proefschrift. Daarnaast wil ik alle coauteurs bedanken voor hun bijdrage aan mijn publicaties en dit proefschrift.

Alle (ex) collega's van de afdeling Kindergeneeskunde ben ik dankbaar voor alle hulp en ondersteuning. De teamspirit, vele koffiepauzes en Musti Fridays houden de moraal altijd hoog en geeft moed tijdens de zware momenten. Lilian, Nico en Leon, jullie ondersteuning is buitengewoon waardevol. Dank jullie wel voor jullie hulp en het enthousiasme voor het brein onderzoek. Ik kijk uit naar onze samenwerking in de komende drie jaren.

Mijn (ex) kamergenoten Matthias, Maria, Ruth, Jip, Monique, Elke, Jennifer, Sizzle en Alex. Beste Ruth, jouw succesvolle stage werd beloond met een promotieplaats. Je mag zeer trots zijn op alles wat je al hebt bereikt en ik wens jou heel veel succes met je verdere carrière. Jip, onze 'vreemde eend in de bijt', dank je wel voor je ondersteuning en je enthousiasme. Ik wens jou heel veel succes met de afronding van je promotie en je verdere carrière als plastisch chirurg. Alex, Monique, Sizzle, Elke en Jennifer, dank jullie wel voor jullie hulp, begeleiding en de luisterende oren. Mijn 'nieuwe' collega's Charlotte, Helene en Luise, wil ik heel veel succes wensen tijdens jullie promotietraject. 
Alle studenten die mij hebben ondersteund tijdens mijn onderzoek, maar ook een grote bijdrage hebben geleverd aan dit proefschrift wil ik bedanken voor hun steun en enthousiasme. Jason, Evelyn, Joël, Eefje, Dennis, Ilona, Lotte en Vera, dank jullie wel en heel veel succes met jullie carrière!

Ook ben ik mijn collega's van de grote proefdiervoorziening zeer dankbaar voor hun toewijding en sublieme assistentie tijdens mijn onderzoek. Sanne, Petra, Joyce, Hub en Saskia dank jullie wel !!!

Naast het lab Kindergeneeskunde, ben ik ook dankbaar voor alle hulp op het laboratorium van Neuroscience. Hellen, Denise, Marian en Marcella, heel hartelijk dank voor jullie hulp en flexibiliteit!

Ook de dames van het secretariaat Kindergeneeskunde verdienen een welgemeend bedankje. Peggy, dank je wel voor alles! Tamara, dank je wel voor je hulp en voor je luisterend oor.

Mijn paranymfen Maria en Matthias wil ik heel hartelijk danken. Ik ben dankbaar dat jullie naast mijn partners in crime in sheep business ook aan mijn zijde willen staan tijdens mijn promotie. Dear Maria, my partner in crime from the very start. We had a lot of fun together, or at least, I did by making jokes about you... Remember Maria in de kantine... or yes yes yes, it's always Maria's fault... I'm really grateful that I can finish this journey together with you, and very thankful for your commitment, optimistic spirit and support. I wish you all the best in the future and hope that science will reunite us. Please, also say a big thank you to your mother for the best spanakopita in the world. Dear Matthias. I'm very grateful you joined our group. Thank you very much for your incredible support and determination during sheep season and our countless coffee breaks. I am happy we remain partners in crime and I look forward to our times on the LICU.

Het is heerlijk om af en toe ongegeneerd te ventileren en af te schakelen. Daarom wil ik de 'oude ballen' Jordi, Paul en Robbert hartelijk danken voor alle gezellige momenten tijdens de trainingen, aan de bar, onze etentjes en tijdens onze wandelingen.

Mijn schoonfamilie, Martha, Jacqueline, Nuray, en Coen. Dank jullie wel voor jullie steun en gezelligheid. Dankzij jullie is Heerlen toch wel mijn thuis geworden.

Lieve pap en mam, Lieke, Tom en Juul. Dank jullie wel voor jullie geduld, vertrouwen en steun. Pap en mam, ik heb jullie geduld menig maal op de proef gesteld door alles vooral op mijn eigen manier te doen. Of sterker nog, door vooral niets te doen... Maar ik heb t toch geflikt.

Yill, geen woorden kunnen beschrijven hoe blij ik ben dat jij in mijn leven bent. Ik heb je geduld meermaals behoorlijk op de proef gesteld met mijn frustraties, maar met jouw geduld en luisterend oor wist je me altijd te kalmeren. Dank je wel! Ik verheug me op alles wat nog op ons pad komt... ik hou van je! 


\section{Curriculum vitae}

Daan Ophelders werd geboren op 8 juli 1983 in Venlo. Hij behaalde zijn VWO diploma in 2002 aan het Valuascollege in Venlo om vervolgens in opleiding te gaan tot operatieassistent in het Maastricht Universitair Medisch Centrum (Academisch ziekenhuis Maastricht). In 2005 behaalde hij zijn diploma en hij heeft tot 2011 als operatieassistent gewerkt in het Maastricht Universitair Medisch. Gedurende zijn werkzaamheden als operatieassistent is hij in 2006 gestart met de Bachelor studie Moleculaire Levenswetenschappen aan de Universiteit Maastricht gevolgd door de Master Molecular Life Science met specialisatie oncologie en ontwikkelingsbiologie waarvoor hij in 2011 afstudeerde bij de afdeling Kindergeneeskunde onder de begeleiding van Dr. Reint Jellema. Zijn afstudeerstage heeft hij gecontinueerd als onderzoeksassistent en vervolgens een promotietraject onder de supervisie van Dr. Tim Wolfs, Dr. Reint Jellema en Prof. Dr. Boris Kramer. De resultaten van het promotieonderzoek zijn in dit proefschrift beschreven. In januari 2017 is hij begonnen als post-doctoraal onderzoeker bij de afdeling Kindergeneeskunde in het MUMC+ onder de supervisie van Dr. Tim Wolfs. Daan Ophelders woont sinds 2010 samen met Yill Raduzzi in Heerlen.

Daan Ophelders was born on July 8, 1983 in Venlo, the Netherlands. In 2002 he graduated from secondary school (Atheneum) at the Valluascollege in Venlo. Subsequently he moved to Maastricht to go into training to become a surgical assistant at the Maastricht University Medical Center (Academic Hospital Maastricht). In 2005 he graduated from his training and he remained working in Maastricht until 2011. During his work in the surgical department, he started in 2006 with the Bachelor study Molecular Life Sciences at Maastricht University, followed by the Master Molecular Life Science with specialization in oncology and developmental biology. In 2011 he obtained his Master's degree after a senior internship at the Department of Pediatrics under the guidance of Dr. Reint Jellema. After graduation for his Master's degree, he started working at the Department of Pediatrics as a research assistant, followed by a PhD trajectory under the supervision of Dr. Tim Wolfs, Dr. Reint Jellema and Prof. Dr. Boris Kramer. The results of his doctoral research described in this thesis. Since January 2017 he works as a post-doctoral researcher in the Department of Pediatrics at the Maastricht University Medical Center+ under the supervision of Dr. Tim Wolf. Daan Ophelders lives together with Yill Raduzzi in Heerlen. 



\section{Publications}

1. Ophelders $D^{*}$, Wolfs $T^{*}$, Jellema RK, Zwanenburg A, Ludwig A, Radtke S. Mesenchymal stromal cellderived extracellular vesicles protect the fetal brain after hypoxia-ischemia. Stem cells translational medicine. 2016;5(6):754-63.

2. Caron MM, Emans PJ, Sanen K, Surtel DA, Cremers A, Ophelders D, et al. The role of prostaglandins and COX-enzymes in chondrogenic differentiation of ATDC5 progenitor cells. PLoS One. 2016;11(4):e0153162.

3. Nikiforou M, Willburger C, de Jong AE, Kloosterboer N, Jellema RK, Ophelders DR, et al. Global Hypoxia-Ischemia Induced Inflammation and Structural Changes in the Preterm Ovine Gut Which Were Not Ameliorated by Mesenchymal Stem Cell Treatment. Molecular Medicine. 2016;22:244.

4. Zwanenburg A, Hermans BJ, Andriessen P, Niemarkt HJ, Jellema RK, Ophelders DR, et al. Comparison of ECG-based physiological markers for hypoxia in a preterm ovine model. Pediatr Res. 2016.

5. Ophelders $D R^{*}$, Gussenhoven $R^{*}$, Lammens $M$, Küsters B, Kemp MW, Newnham JP, et al. Neuroinflammation and structural injury of the fetal ovine brain following intra-amniotic Candida albicans exposure. Journal of neuroinflammation. 2016;13(1):1.

6. Willems MG, Ophelders DR, Nikiforou M, Jellema RK, Butz A, Delhaas T, et al. Systemic interleukin-2 administration improves lung function and modulates chorioamnionitis-induced pulmonary inflammation in the ovine fetus. American Journal of Physiology-Lung Cellular and Molecular Physiology. 2016;310(1):L1-L7.

7. Jellema RK*, Ophelders DR ${ }^{*}$, Zwanenburg A, Nikiforou M, Delhaas T, Andriessen P, et al. Multipotent adult progenitor cells for hypoxic-ischemic injury in the preterm brain. Journal of neuroinflammation. 2015;12(1):241.

8. Hütten MC, Kuypers E, Ophelders DR, Nikiforou M, Jellema RK, Niemarkt HJ, et al. Nebulization of Poractant alfa via a vibrating membrane nebulizer in spontaneously breathing preterm lambs with binasal continuous positive pressure ventilation. Pediatr Res. 2015;78(6):664-9.

9. Hütten MC, Goos TG, Ophelders D, Nikiforou M, Kuypers E, Willems M, et al. Fully automated predictive intelligent control of oxygenation (PRICO) in resuscitation and ventilation of preterm lambs. Pediatr Res. 2015. 
10. Nikiforou M, Vanderlocht J, Chougnet CA, Jellema RK, Ophelders DR, Joosten M, et al. Prophylactic interleukin-2 treatment prevents fetal gut inflammation and injury in an ovine model of chorioamnionitis. Inflammatory bowel diseases. 2015;21(9):2026-38.

11. Niemarkt HJ, Kuypers E, Jellema R, Ophelders D, Hütten $M$, Nikiforou $M$, et al. Effects of less-invasive surfactant administration on oxygenation, pulmonary surfactant distribution, and lung compliance in spontaneously breathing preterm lambs. Pediatr Res. 2014;76(2):166-70.

12. Kuypers E, Jellema RK, Ophelders DR, Dudink J, Nikiforou M, Wolfs TG, et al. Effects of intra-amniotic lipopolysaccharide and maternal betamethasone on brain inflammation in fetal sheep. PLoS One. 2013;8(12):e81644.

13. Schoberer M, Arens J, Erben A, Ophelders D, Jellema RK, Kramer BW, et al. Miniaturization: the clue to clinical application of the artificial placenta. Artificial organs. 2014;38(3):208-14.

14. Jellema RK, Passos VL, Ophelders DR, Wolfs TG, Zwanenburg A, De Munter S, et al. Systemic G-CSF attenuates cerebral inflammation and hypomyelination but does not reduce seizure burden in preterm sheep exposed to global hypoxia-ischemia. Experimental neurology. 2013;250:293-303.

15. Jellema RK, Wolfs TG, Passos VL, Zwanenburg A, Ophelders DR, Kuypers E, et al. Mesenchymal stem cells induce T-cell tolerance and protect the preterm brain after global hypoxia-ischemia. 2013.

16. Jellema RK, Lima Passos V, Zwanenburg A, Ophelders D, De Munter S, Vanderlocht J, et al. Cerebral inflammation and mobilization of the peripheral immune system following global hypoxia-ischemia in preterm sheep. J Neuroinflammation. 2013;10(13):2094-10.

17. Zwanenburg A, Jellema RK, Jennekens W, Ophelders D, Vullings R, van Hunnik A, et al. Heart rate-mediated blood pressure control in preterm fetal sheep under normal and hypoxic-ischemic conditions. Pediatr Res. 2013;73(4-1):420-6.

18. Seehase M, Collins JJ, Kuypers E, Jellema RK, Ophelders DR, Ospina OL, et al. New surfactant with SP-B and $C$ analogs gives survival benefit after inactivation in preterm lambs. PLoS One. 2012;7(10):e47631.

19. Kuypers E, Ophelders D, Jellema RK, Kunzmann S, Gavilanes AW, Kramer BW. White matter injury following fetal inflammatory response syndrome induced by chorioamnionitis and fetal sepsis: lessons from experimental ovine models. Early human development. 2012;88(12):931-6. 
